\title{
Gordon's Conjectures 1 and 2: Pontryagin-van Kampen duality in the hyperfinite setting
}

\author{
PAVOL Zlatoš
}

\begin{abstract}
Using the ideas of E. I. Gordon we present and further advance an approach, based on nonstandard analysis, to simultaneous approximations of locally compact abelian groups and their duals by (hyper)finite abelian groups, as well of the Haar measures on them. Combining the techniques of nonstandard analysis and the Fourier analytic methods of additive combinatorics we prove the first two of the three Gordon's Conjectures which were open since 1991 and are crucial both in the formulations and proofs of the approximation theorems for LCA groups and for the Fourier transform.
\end{abstract}

2010 Mathematics Subject Classification 22B05 (primary); 03H05, 26E35, 28E05, 54J05, 65T50 (secondary)

Keywords: Fourier transform, nonstandard analysis, hyperfinite, infinitesimal approximation, locally compact abelian group, Pontryagin-van Kampen duality

\section{(Introduction}

Locally compact abelian groups (briefly, LCA groups) and the Pontryagin-van Kampen Duality Theorem provide a general background on which all the particular instances of the (commutative) Fourier transform can be treated in a uniform way (see, eg, Hewitt and Ross [24, 25], Loomis [30], and Rudin [42]). At the same time, the existence of very fast algorithms for the Fourier transform of functions ( $n$-dimensional vectors) $f: \mathbb{Z}_{n} \rightarrow \mathbb{C}$ and, more generally, of functions $f: G \rightarrow \mathbb{C}$ defined on an arbitrary finite abelian group $G$, calls for their systematic employment in approximating the Fourier transforms on various functional spaces over general LCA groups. This is indeed the case in many important particular cases, including the Fourier series of periodic functions $f: \mathbb{R} \rightarrow \mathbb{C}$ with a fixed period $T>0$, the Fourier transform of functions $f: \mathbb{R} \rightarrow \mathbb{C}$ and $f: \mathbb{R}^{n} \rightarrow \mathbb{C}$, the semidiscrete Fourier transform of sequences $f: \mathbb{Z} \rightarrow \mathbb{C}$, etc (see, eg, Epstein [11], Gröchenig [21], and Terras [45]).

The general situation requires us to elaborate the concepts and techniques of approximation of arbitrary LCA groups by finite abelian groups in the first step. In the second 
step it is necessary to be able to approximate simultaneously an LCA group $\mathbf{G}$ and its dual group $\widehat{\mathbf{G}}$ by some finite abelian group $G$ and its dual group $\widehat{G}$, respectively, in such in a way that the pairing $(\boldsymbol{x}, \gamma) \mapsto \gamma(\boldsymbol{x})$ on $\mathbf{G} \times \widehat{\mathbf{G}}$ is approximated by the pairing $(x, \gamma) \mapsto \gamma(x)$ on $G \times \widehat{G}$. Only then we have a chance to succeed with the third and final step, ie to approximate the Fourier transform, say $\mathbf{F}: \mathrm{L}^{1}(\mathbf{G}) \rightarrow \mathrm{C}_{0}(\widehat{\mathbf{G}})$, by the discrete Fourier transform $\mathcal{F}: \mathbb{C}^{G} \rightarrow \mathbb{C}^{\widehat{G}}$.

In this paper we focus on the first two of the three steps mentioned above. The approximation of the Fourier transform on various Banach spaces of functions defined on general LCA groups will be studied in our subsequent paper [50].

This paper makes systematic use of nonstandard analysis (NSA), both as a rigorous method as well as a source of insights, inspiration and intuitively appealing concepts. In the remainder of this section, in which we review the main results of the paper, we assume at least a brief acquaintance with some concepts and results of NSA, as well as of the duality theory for LCA groups and of the Fourier transform. A more complete presentation is given in the main body of the paper.

NSA will makes it possible to "compress" a whole system of increasingly refined finite approximations "converging" to some LCA group $\mathbf{G}$ into a single nicely structured object $\left(G, G_{0}, G_{\mathrm{f}}\right)$, called a bounded monadic group, consisting of a hyperfinite abelian group $G$ with internal group operations, and its two external subgroups $G_{0} \subseteq G_{\mathrm{f}}$, with $G_{0}$ monadic and $G_{f}$ galactic. Alternatively, and even more frequently, bounded monadic groups will referred to as $I M G$ group triplets ( $I$ for internal, $M$ for monadic, $G$ for galactic). Intuitively, $G_{0}$ is viewed as the subgroup of infinitesimals and $G_{\mathrm{f}}$ is viewed as the subgroup of finite elements of $G$. In the simplest, but important, particular case, $G_{0}$ is the intersection and $G_{\mathrm{f}}$ is the union of countably many internal sets. Then the quotient $G^{b}=G_{\mathrm{f}} / G_{0}$, obtained by restricting to the finite elements and identifying those of them which are indistinguishable, is called the observable trace of the triplet $\left(G, G_{0}, G_{\mathrm{f}}\right)$. For any $x \in G_{\mathrm{f}}$ the coset $x^{b}=x+G_{0} \in G^{b}$ is called the observable trace or monad of $x$. Our IMG triplets will additionally be condensing, which property is equivalent to the condition that, for any internal sets $A, B$ such that $G_{0} \subseteq A \subseteq B \subseteq G_{\mathrm{f}}$, the quotient $|B| /|A|$ is finite.

The equivalence relation of infinitesimal nearness or indistinguishability on $G$, given by

$$
x \approx y \Leftrightarrow x-y \in G_{0}
$$

induces in a fairly natural way a topology on the observable trace $G^{b}$, turning it into a (Hausdorff) locally compact group. Conversely, every LCA group $\mathbf{G}$ is isomorphic (as a topological group) to the observable trace $G^{b}=G_{\mathrm{f}} / G_{0}$ of some condensing 
IMG group triplet $\left(G, G_{0}, G_{\mathrm{f}}\right)$ with a hyperfinite abelian ambient group $G$. In standard terms this means that every LCA group $\mathbf{G}$ can be "arbitrarily well" approximated by finite abelian groups in a sense made precise by a definition introduced by Gordon [17] (see Section 1.3, particularly Proposition 1.3.7, Theorem 1.3.10, and - mainly Corollary 1.3.11 for more details). For compact metrizable groups this approximability concept coincides with that already studied by Alan Turing [46] in the late 1930s (see the second remark following the proof of Proposition 1.3.7).

For an LCA group $\mathbf{G}$, its dual group $\widehat{\mathbf{G}}=\operatorname{Hom}_{\mathrm{c}}(\mathbf{G}, \mathbb{T})$ consists of all continuous homomorphisms (characters) $\gamma: \mathbf{G} \rightarrow \mathbb{T}$, where $\mathbb{T}$ denotes the compact multiplicative group of complex units. Then $\widehat{\mathbf{G}}$, endowed with the componentwise multiplication and the compact-open topology becomes an LCA group again. The Pontryagin-van Kampen Duality Theorem states that the canonical mapping $\mathbf{G} \rightarrow \widehat{\widehat{\mathbf{G}}}$, sending any $\boldsymbol{x} \in \mathbf{G}$ to the character $\widehat{\boldsymbol{x}}: \widehat{\mathbf{G}} \rightarrow \mathbb{C}$ of the dual group $\widehat{\mathbf{G}}$, given by the pairing $\widehat{\boldsymbol{x}}(\gamma)=\gamma(\boldsymbol{x})$ on $\mathbf{G} \times \widehat{\mathbf{G}}$, is an isomorphism of topological groups (see, eg, Dikranjan and Stoyanov [9], Hewitt and Ross [24], Morris [36], Pontryagin [38] or Rudin [42]).

For a finite abelian group $G$, its dual group $\widehat{G}=\operatorname{Hom}(G, \mathbb{T})$ consists of all homomorphisms $\gamma: G \rightarrow \mathbb{T}$, and it is isomorphic (though not canonically) to $G$. The fact that the canonical mapping $G \rightarrow \widehat{\widehat{G}}$ is an isomorphism of (discrete) abelian groups follows immediately. For a hyperfinite abelian group $G$ we denote by $\widehat{G}=\operatorname{Hom}\left(G,{ }^{*} \mathbb{T}\right)$ the set of all internal homomorphisms (characters) $\gamma: G \rightarrow{ }^{*} \mathbb{T}$. According to the Transfer Principle, $\widehat{G}$ with the pointwise multiplication is a hyperfinite abelian group internally (though not canonically) isomorphic to $G$, and the canonical mapping $G \rightarrow \widehat{\widehat{G}}$ is an internal group isomorphism.

Assuming that $\left(G, G_{0}, G_{\mathrm{f}}\right)$ is a condensing IMG group triplet with a hyperfinite abelian ambient group $G$, let us denote by $\mathbf{G}=G^{b}=G_{\mathrm{f}} / G_{0}$ its observable trace. Then its dual $\widehat{\mathbf{G}}$ is an LCA group, too, hence it is isomorphic to the observable trace $H^{b}=H_{\mathrm{f}} / H_{0}$ of some condensing IMG group triplet $\left(H, H_{0}, H_{\mathrm{f}}\right)$ with a hyperfinite abelian ambient group $H$. One can naturally expect that the triplet representing the dual group $\widehat{\mathbf{G}}$ can be constructed from the original triplet $\left(G, G_{0}, G_{\mathrm{f}}\right)$ in some canonical way. The starting point is the natural requirement that $H=\widehat{G}$. Then the subsets

$$
\begin{aligned}
& G_{0}^{\downarrow}=\left\{\gamma \in \widehat{G}:\left(\forall x \in G_{0}\right)(\gamma(x) \approx 1)\right\} \\
& G_{\mathrm{f}}^{\downarrow}=\left\{\gamma \in \widehat{G}:\left(\forall x \in G_{\mathrm{f}}\right)(\gamma(x) \approx 1)\right\}
\end{aligned}
$$

of the dual group $\widehat{G}$, called the infinitesimal annihilators of the subgroups $G_{0}, G_{\mathrm{f}}$, respectively, are subgroups of $\widehat{G}$. It can be shown that $\left(\widehat{G}, G_{\mathrm{f}}^{\downarrow}, G_{0}^{\downarrow}\right)$ is a condensing IMG group triplet with a hyperfinite abelian ambient group $\widehat{G}$ (see Proposition 2.2.2). 
The characters $\gamma \in G_{\mathrm{f}}^{\downarrow}$ are infinitesimally close to 1 on the whole subgroup $G_{\mathrm{f}}$ of finite elements of $G$. They play the role of infinitesimal characters. As every $\gamma \in \widehat{G}$ is a group homomorphism, it belongs to $G_{0}^{\sim}$ if and only if it is $S$-continuous as a mapping $\gamma: G \rightarrow{ }^{*} \mathbb{T}$. The elements of $G_{0}^{\downarrow}$ play the role of finite characters.

The condensing IMG group triplet $\left(\widehat{G}, G_{\mathrm{f}}^{\downarrow}, G_{0}^{\downarrow}\right)$ will be called the dual triplet of the IMG group triplet $\left(G, G_{0}, G_{\mathrm{f}}\right)$.

For an internal character $\gamma \in G_{0}^{\downarrow}$, its observable trace $\gamma^{b}$ can be considered as the element $G_{\mathrm{f}}^{\downarrow} \gamma$ of the quotient $G_{0}^{\downarrow} / G_{\mathrm{f}}^{\downarrow}=\widehat{G}^{b}$, as well as the observable trace $\gamma^{b}$ of the $S$-continuous mapping $\gamma: G \rightarrow{ }^{*} \mathbb{T}$, ie

$$
\gamma^{b}\left(x^{b}\right)={ }^{\circ} \gamma(x)
$$

for $x \in G_{\mathrm{f}}$. That way $\gamma^{b}: G^{b} \rightarrow \mathbb{T}$ is a continuous character of the LCA group $G^{b}$ (see Section 1.3). The assignment $\gamma \mapsto \gamma^{b}$, depicted in the commutative diagram



is a group homomorphism $G_{0}^{\downarrow} \rightarrow \widehat{G^{b}}$. Its kernel is the subgroup $G_{\mathrm{f}}^{\downarrow} \subseteq G_{0}^{\downarrow}$ of all infinitesimal characters in $\widehat{G}$. Thus the assignment $\gamma \mapsto \gamma^{b}$ induces an injective group homomorphism $\widehat{G}^{b} \rightarrow \widehat{G^{b}}$ from the observable trace $\widehat{G}^{b}=G_{0}^{\downarrow} / G_{\mathrm{f}}^{\downarrow}$ of the dual triplet $\left(\widehat{G}, G_{\mathrm{f}}^{\downarrow}, G_{0}^{\downarrow}\right)$ into the dual group $\widehat{G^{b}}=\widehat{G_{\mathrm{f}} / G_{0}}$ of the observable trace $G^{b}=G_{\mathrm{f}} / G_{0}$ of the original triplet $\left(G, G_{0}, G_{\mathrm{f}}\right)$. The canonical injective homomorphism $G_{0}^{\nu} / G_{\mathrm{f}}^{\downarrow} \rightarrow \widehat{G_{\mathrm{f}} / G_{0}}$ justifies the identification of the "two observable traces" $G_{\mathrm{f}}^{\nu} \gamma$ and $\gamma^{b}$. As proved by Gordon in [17] (see also [18]), the assignment $G_{\mathrm{f}}^{\downarrow} \gamma \mapsto \gamma^{b}$ is an isomorphism of the topological group $\widehat{G}^{b}=G_{0}^{\downarrow} / G_{\mathrm{f}}^{\downarrow}$ onto a closed subgroup of the topological group $\widehat{G^{b}}=\widehat{G_{\mathrm{f}} / G_{0}}$.

It is both tempting and natural to conjecture that the canonical mapping $\widehat{G}^{b} \rightarrow \widehat{G^{b}}$ is also surjective, ie, that it is an isomorphism of topological groups. This is indeed the first of Gordon's Conjectures from [17] (see also [18, page 132]), which we will prove as our Theorem 2.2.4.

Gordon's Conjecture 1 Let $\left(G, G_{0}, G_{\mathrm{f}}\right)$ be a condensing IMG group triplet with a hyperfinite abelian ambient group $G$. Then the canonical mapping $G_{0}^{\downarrow} / G_{\mathrm{f}}^{\downarrow} \rightarrow \widehat{G_{\mathrm{f}} / G_{0}}$ is an isomorphism of topological groups. 
The proof of this conjecture amounts just to show that every continuous character $\gamma$ of the LCA group $G^{b}=G_{\mathrm{f}} / G_{0}$ is of the form $\gamma=\gamma^{b}$ for some internal $S$-continuous character $\gamma \in G_{0}^{\downarrow}$. We will reach this goal in a roundabout way by investigating the dual triplet of the dual triplet of the original IMG group triplet $\left(G, G_{0}, G_{\mathrm{f}}\right)$.

The second dual $\widehat{\widehat{G}}$ of the hyperfinite abelian group $G$ can be naturally identified with the original group $G$. Then the second dual of the original triplet $\left(G, G_{0}, G_{\mathrm{f}}\right)$ is defined as the condensing IMG triplet $\left(G, G_{0}^{\downarrow \downarrow}, G_{\mathrm{f}}^{\downarrow \downarrow}\right)$. As we shall see in Sections 2.2 and 2.3, Gordon's Conjecture 1 is equivalent to the following statement, which we will prove as our Theorem 2.2.5.

The Triplet Duality Theorem Let $\left(G, G_{0}, G_{\mathrm{f}}\right)$ be a condensing IMG group triplet with a hyperfinite abelian ambient group $G$. Then:

$$
G_{0}^{\downarrow \downarrow}=G_{0} \quad \text { and } \quad G_{\mathrm{f}}^{\downarrow \downarrow}=G_{\mathrm{f}}
$$

In other words, the dual triplet $\left(G, G_{0}^{\downarrow \sim}, G_{\mathrm{f}}^{\downarrow \downarrow}\right)$ of the dual triplet $\left(\widehat{G}, G_{\mathrm{f}}^{\downarrow}, G_{0}^{\downarrow}\right)$ equals the original group triplet $\left(G, G_{0}, G_{\mathrm{f}}\right)$.

The first equality $G_{0}^{\downarrow} \downarrow=G_{0}$, proved in Proposition 2.3.3, is crucial. The second equality $G_{\mathrm{f}}^{\downarrow} \downarrow=G_{\mathrm{f}}$ follows from it and the fact that $G_{\mathrm{f}}^{\downarrow \nu}=G_{\mathrm{f}}+G_{0}^{\downarrow \nu}$, proved as Lemma 2.2.7. As proved by Gordon ([17, Theorem 3.2] and [18, Theorem 2.2.18]), even the weaker equality $G_{0}=G_{0}^{\downarrow} \sim \cap G_{\mathrm{f}}$ implies Gordon's Conjecture 1 .

Given a condensing IMG group triplet $\left(G, G_{0}, G_{\mathrm{f}}\right)$ with a hyperfinite ambient group $G$, a positive number $d \in{ }^{*} \mathbb{R}$ is called a normalizing multiplier or a normalizing coefficient for $\left(G, G_{0}, G_{\mathrm{f}}\right)$ if the number $d|A|$ is finite and noninfinitesimal for some (or, equivalently, for each) internal set $A$ between $G_{0}$ and $G_{\mathrm{f}}$. In particular, for any internal set $A$ between $G_{0}$ and $G_{\mathrm{f}}, d=1 /|A|$ is a normalizing coefficient for $\left(G, G_{0}, G_{\mathrm{f}}\right)$.

If $d$ is any normalizing multiplier for $\left(G, G_{0}, G_{\mathrm{f}}\right)$ then the Loeb measure $\lambda_{d}$ induced by the constant function $d(x)=d$ on $G$ is translation invariant. The measure $\boldsymbol{m}_{d}$ obtained by "pushing down" the Loeb measure $\lambda_{d}$ is the Haar measure on the observable trace $G^{b}=G_{\mathrm{f}} / G_{0}$ (see Proposition 1.3.6, as well as the final part of Section1.2 and the references there). Thus in order to approximate the invariant integration on the observable trace $G^{b}$ by hyperfinite sums of internal functions $G \rightarrow{ }^{*} \mathbb{C}$, the inner product on the space ${ }^{*} \mathbb{C}^{G}$ has to be normalized by some normalizing multiplier $d$, ie

$$
\langle f, g\rangle_{d}=d \sum_{x \in G} f(x) \overline{g(x)}
$$


for $f, g \in{ }^{*} \mathbb{C}^{G}$. In particular, the Fourier transform of a function $f \in{ }^{*} \mathbb{C}^{G}$ has to be defined by

$$
\widehat{f}(\gamma)=\langle f, \gamma\rangle_{d}=d \sum_{x \in G} f(x) \overline{\gamma(x)}
$$

for $\gamma \in \widehat{G}$. Then, in order to get the Fourier inversion formula and the Plancherel identity, we need to normalize the inner product and the Fourier transform on the space ${ }^{*} \mathbb{C}^{\widehat{G}}$ of all internal functions $\widehat{G} \rightarrow{ }^{*} \mathbb{C}$ by means of the coefficient $\widehat{d}=1 / d|G|$.

In view of the canonical isomorphism of the observable trace $\widehat{G}^{b}=G_{0}^{\downarrow} / G_{\mathrm{f}}^{\downarrow}$ and the dual group $\widehat{G^{b}}=\widehat{G_{\mathrm{f}} / G_{0}}$, there naturally arises the question whether such a $\widehat{d}$ is indeed a normalizing multiplier for the dual triplet $\left(\widehat{G}, G_{\mathrm{f}}^{\downarrow}, G_{0}^{\downarrow}\right)$. In that case (and only in that case) the measure $\boldsymbol{m}_{\widehat{d}}$, obtained by pushing down the Loeb measure $\lambda_{\widehat{d}}$ from $\widehat{G}$ to the observable trace $\widehat{G}^{b} \cong \widehat{G^{b}}$, will be a Haar measure on $\widehat{G}^{b}$. The second of Gordon's conjectures, proved as our Theorem 2.3.4, states that the response to this question is affirmative.

Gordon's Conjecture 2 Let $\left(G, G_{0}, G_{\mathrm{f}}\right)$ be a condensing IMG group triplet with a hyperfinite abelian ambient group $G$. Then, for any internal set $D$ such that $G_{0} \subseteq D \subseteq G_{\mathrm{f}}, d=1 /|D|$ is a normalizing multiplier for $\left(G, G_{0}, G_{\mathrm{f}}\right)$ and $\widehat{d}=|D| /|G|$ is a normalizing multiplier for the dual triplet $\left(\widehat{G}, G_{\mathrm{f}}^{\downarrow}, G_{0}^{\downarrow}\right)$. More generally, if $d$ is any normalizing multiplier for $\left(G, G_{0}, G_{\mathrm{f}}\right)$ then $\widehat{d}=(d|G|)^{-1}$ is a normalizing multiplier for $\left(\widehat{G}, G_{\mathrm{f}}^{\downarrow}, G_{0}^{\downarrow}\right)$.

The methods of nonstandard analysis were applied for the first time in the study of the Fourier series of functions $\mathbb{T} \rightarrow \mathbb{C}$ by Luxemburg [32]. His key idea consisted in embedding the group of integers $\mathbb{Z}$ into the hyperfinite cyclic group $\mathbb{Z}_{n}$, where $n \in{ }^{*} \mathbb{N} \backslash \mathbb{N}$, and an infinitesimal approximation of the group of complex units $\mathbb{T}$ by the hyperfinite subgroup $\left\{\mathrm{e}^{2 \pi \mathrm{i} k / n}: k \in \mathbb{Z}_{n}\right\} \cong \mathbb{Z}_{n}$ of its nonstandard extension ${ }^{*} \mathbb{T}$. The first treatment of abstract (commutative) harmonic analysis by nonstandard methods in full generality is due to Gordon. In a series of works culminating in $[17,18]$ he elaborated a nonstandard approach to approximations of LCA groups by hyperfinite abelian groups, formulated a version of Pontryagin-van Kampen duality for them and developed an approach to the approximation of the classical Fourier-Plancherel transform $\mathrm{L}^{2}(\mathbf{G}) \rightarrow \mathrm{L}^{2}(\widehat{\mathbf{G}})$ by the discrete Fourier transform on the approximating hyperfinite group. At the same time, he formulated three fairly fundamental conjectures in [17] which remained open until 2012. In fact, Gordon accepted one not very essential restriction: having worked in a nonstandard universe which was only assumed to be $\aleph_{1}$-saturated, he was forced to deal just with triplets $\left(G, G_{0}, G_{\mathrm{f}}\right)$ in which $G_{0}$ and $G_{\mathrm{f}}$ 
were the intersection and the union, respectively, of countably many internal sets. As a consequence, the class of LCA groups $\mathbf{G}$ representable as observable traces $\mathbf{G} \cong G_{\mathrm{f}} / G_{0}$ of such triplets was reduced to the metrizable $\sigma$-compact ones.

Gordon even proved all his three conjectures for any such a triplet $\left(G, G_{0}, G_{\mathrm{f}}\right)$ having an internal subgroup $K$ such that $G_{0} \subseteq K \subseteq G_{\mathrm{f}}$. In standard terms this corresponds to the situation that the LCA group $\mathbf{G}=G_{\mathrm{f}} / G_{0}$ contains a compact open subgroup. Then, using the fact that the fairly natural triplet representing the LCA group $\mathbb{R}$ (see Example 1.3.8(c)) satisfies these conjectures as well, and the structure theorem for LCA groups - according to which every LCA group $\mathbf{G}$ is isomorphic to the product $\mathbf{H} \times \mathbb{R}^{n}$ for some LCA group $\mathbf{H}$ containing a compact open subgroup and some $n \in \mathbb{N}$ - he was able to prove that every (metrizable $\sigma$-compact) LCA group $\mathbf{G}$ is isomorphic to the observable trace $G_{\mathrm{f}} / G_{0}$ of some condensing IMG group triplet with an abelian hyperfinite ambient group $G$, satisfying all the three conjectures.

In the present paper we will recapitulate Gordon's approach and some of his results, introducing several conceptual and notational modifications, based partly on Ziman and Zlatoš [47], and prove the first two of his conjectures for arbitrary condensing IMG triplets $\left(G, G_{0}, G_{\mathrm{f}}\right)$ with an abelian hyperfinite ambient group $G$. What's of minor importance, working in a "sufficiently saturated" nonstandard universe, our triplets will represent all (Hausdorff) LCA groups (not just the metrizable $\sigma$-compact ones) as their observable traces. Besides the techniques of nonstandard analysis and harmonic analysis, the crucial role in our proofs will be played by the Fourier analytic methods of additive combinatorics due mainly to Green and Ruzsa [20], and Tao and Vu [44] (see Section 2.1). So in this respect we almost will not build on Gordon's work at all; in particular, our proofs will not rely upon the above mentioned structure theorem for LCA groups. Finally, we will present some in standard terms formulated equivalents of most of the mentioned nonstandard results. However, as we shall see, several of these standard formulations (even in the metrizable $\sigma$-compact case) tend to become rather complicated and lack the intuitive transparency of their nonstandard counterparts.

Acknowledgements. The author acknowledges with thanks the support of his research by the grants no 1/0608/13 and 1/0333/17 of the Slovak Scientific Grant Agency VEGA.

At the same time I would like to express my deep indebtedness and gratitude to Zhenya Gordon for having introduced me to the topic of the nonstandard approach to Pontryagin-van Kampen duality and the Fourier transform, as well as for many valuable discussions and permanent encouragement and support when my long year wrestling with his Conjectures seemed desperately hopeless.

This paper is dedicated to the memory of Petr Vopěnka (1935-2015). 


\section{Nonstandard analysis}

The reader is assumed to have some basic acquaintance with nonstandard analysis, including the nonstandard approach to topology and continuity in terms of monads and equivalence relations of infinitesimal nearness, and the Loeb measure construction. Besides the original Robinson's book [39], the standard general references include the monographs by Albeverio, Fenstad, Høegh-Krohn and Lindstrøm [1], Arkeryd, Cutland and Henson [4] (mainly the parts by Henson [23], Loeb [29] and Ross [41]), Davis [8], and Goldblatt [14]. For Loeb measure also the survey by Cutland [7] can be consulted. Additionally, we refer to Chang and Keisler [6] for the ultraproduct construction and its use in constructing nonstandard extensions of first-order structures and superstructures.

\subsection{General setting}

Our exposition takes place in a nonstandard universe ${ }^{*} \mathbb{V}$ which is an elementary extension of a superstructure $\mathbb{V}$ over some set of individuals containing at least all (classical) complex numbers and the elements of the topological space or topological group, as well as index sets, etc, dealt with.

In particular, every standard mathematical (first-order) structure $A \in \mathbb{V}$ is embedded into its nonstandard extension ${ }^{*} A \in{ }^{*} \mathbb{V}$ via the mapping $a \mapsto{ }^{*} a: A \rightarrow{ }^{*} A$ such that, for any formula $\Phi\left(x_{1}, \ldots, x_{n}\right)$ in the language of $A$ and elements $a_{1}, \ldots, a_{n} \in A$, $\Phi\left(a_{1}, \ldots, a_{n}\right)$ is satisfied in $A$ if and only if $\Phi\left({ }^{*} a_{1}, \ldots,{ }^{*} a_{n}\right)$ is satisfied in ${ }^{*} A$ (the Transfer Principle). Whenever there threatens no confusion we tend to identify $a \in A$ with ${ }^{*} a \in{ }^{*} A$, and to denote the corresponding operations and relations in $A$ and ${ }^{*} A$ by the same sign, dropping ${ }^{*}$ in the latter. Similarly, to a function $f: A \rightarrow B$ in $\mathbb{V}$ there (functorially) corresponds a function ${ }^{*} f:{ }^{*} A \rightarrow{ }^{*} B$ in ${ }^{*} \mathbb{V}$, etc. The nonstandard universe $* \mathbb{V}$ can be constructed as a certain ultrapower of the superstructure $\mathbb{V}$ with respect to some properly chosen ultrafilter.

In particular, we have the structures of hypernatural numbers $* \mathbb{N}$, hyperintegers $* \mathbb{Z}$, hyperrational numbers $* \mathbb{Q}$, hyperreal numbers $* \mathbb{R}$, and hypercomplex numbers $* \mathbb{C}$ with the usual (and, possibly, some additional) operations and relations, extending the structures of natural numbers $\mathbb{N}$, integers $\mathbb{Z}$, rational numbers $\mathbb{Q}$, real numbers $\mathbb{R}$, and complex numbers $\mathbb{C}$, respectively.

As the superstructure $\mathbb{V}$ is transitive, ie $X \subseteq \mathbb{V}$ for any set $X \in \mathbb{V}$, the same is true for $* \mathbb{V}$. The sets belonging to ${ }^{*} \mathbb{V}$ are called internal; other subsets of $* \mathbb{V}$ are called external. Additionally, we assume that the nonstandard universe $* \mathbb{V}$ is either $\kappa$-saturated for 
some uncountable cardinal $\kappa$ or even polysaturated, ie $\kappa$-saturated for some $\kappa$ bigger than the cardinality of any set in the original (standard) universe $\mathbb{V}$. However, for the sake of generality, we do not specify the saturation degree $\kappa$ explicitly. Instead we use the term a set or a system of admissible size referring to (external) subsets of the nonstandard universe with the (external) cardinality $<\kappa$, and assume that the universe $* \mathbb{V}$ is sufficiently saturated, meaning that $\bigcap \mathcal{S} \neq \emptyset$ for any system of internal sets $\mathcal{S} \subseteq{ }^{*} \mathbb{V}$ of admissible size with the finite intersection property. "Sufficiently saturated" universes can be constructed as ultrapowers with respect to "sufficiently good" ultrafilters (see Chang and Keisler [6]). For most applications, however, an $\aleph_{1}$-saturated nonstandard universe (ie, $\kappa=\aleph_{1}$ ) is sufficient; in that case a system of admissible size is simply a countable one.

Internal sets $A$ which can be put into a one-to-one correspondence via an internal bijection with sets of the form $\{1, \ldots, n\}$ for some $n \in{ }^{*} \mathbb{N}$ are called hyperfinite; in that case $n=|A|$ is referred to as the number of elements of $A$. Hyperfinite sets (briefly, HF sets) behave within the internal context much like finite sets, though for $n \in{ }^{*} \mathbb{N} \backslash \mathbb{N}$ they are (externally) infinite.

Internal sets, particularly hyperfinite sets, are the simplest objects in the descriptive settheoretic hierarchy. Next to them in this hierarchy there are the galactic or $\Sigma_{1}^{0}(\kappa)$-sets and the monadic or $\Pi_{1}^{0}(\kappa)$-sets, ie, unions and intersections, respectively, of collections of internal sets of admissible size. From the formal point of view, it is these sets which enable the techniques of Nonstandard Analysis based on saturation. In particular, if $\mathcal{S}$ is any collection of admissible size with the finite intersection property consisting of monadic sets $X \subseteq * \mathbb{V}$ then $\bigcap \mathcal{S} \neq \emptyset$.

An example of a galactic set is the set $\mathbb{N}$ of all (standard) natural numbers, in other words, the set of all finite elements of the internal set of all hypernatural numbers $* \mathbb{N}$. Then the infinite hypernatural numbers form the monadic set ${ }^{*} \mathbb{N}_{\infty}={ }^{*} \mathbb{N} \backslash \mathbb{N}$.

Further typical and fairly important examples of monadic sets are the equivalence relations of indistinguishability or infinitesimal nearness, arising in nonstandard models of metric, uniform and (sufficiently regular) topological spaces, as well as the monads (ie, cosets) of particular elements with respect to such equivalence relations.

Every function $f: X \rightarrow Y$ is considered to be equal to the set of ordered pairs $\{(x, f(x)): x \in X\}$. If $R \subseteq X \times Y$ is a relation then a function $f$ is called a choice function from $R$ on a set $A \subseteq \operatorname{dom} R$ if $A \subseteq \operatorname{dom} f$ and $f\lceil A \subseteq R$, ie, if $(a, f(a)) \in R$ for each $a \in A$. The following lemma describes an important class of relations admitting internal choice functions. 
Lemma 1.1.1 (The Internal Choice Lemma) Let $X, Y$ be internal sets in a sufficiently saturated nonstandard universe, and $R \subseteq X \times Y$ be a relation such that for every internal set $D \subseteq \operatorname{dom} R$ the restriction $R\lceil D$ is a monadic set. Then for every galactic set $A \subseteq \operatorname{dom} R$ there exists an internal choice function $f$ from $R$ on $A$.

Sketch of proof If $A$ is internal then the monadic relation $R\lceil A$ can be written as the intersection $R\left\lceil A=\bigcap_{i \in I} R_{i}\right.$ of admissibly many internal relations $R_{i} \subseteq X \times Y$ with common domain $A$. Then, for any nonempty finite set $J \subseteq I$, we readily obtain an internal choice function $f_{J}$ on $A$ from the relation $\bigcap_{i \in J} R_{i}$, by applying the Transfer Principle to the Axiom of Choice. (For hyperfinite $A$ the Axiom of Choice is not even needed at this point.) The existence of an internal choice function $f$ from $R$ on $A$ follows as a consequence of saturation.

If $A$ is a galactic set then the desired conclusion follows from the internal case by applying the saturation argument once again.

\subsection{Bounded monadic spaces}

For the notion of a uniform space and related notions like uniformity, entourage, uniform continuity, etc, we refer the reader to the monograph by Engelking [10].

If $(\mathbf{X}, \mathcal{U})$ is a uniform space then the relation of infinitesimal nearness or indistinguishability defined by

$$
\mathbf{E}_{\mathcal{U}}=\bigcap_{\mathbf{U} \in \mathcal{U}}{ }^{*} \mathbf{U}
$$

is a monadic equivalence relation on the nonstandard extension ${ }^{*} \mathbf{X}$ of $\mathbf{X}$ (provided the respective nonstandard universe is sufficiently saturated). Then the uniformity $\mathcal{U}$ consists precisely of those subsets $\mathbf{U}$ of $\mathbf{X} \times \mathbf{X}$ satisfying $\mathbf{E}_{\mathcal{U}} \subseteq{ }^{*} \mathbf{U}$. Instead of $(\boldsymbol{x}, \boldsymbol{y}) \in \mathbf{E}_{\mathcal{U}}$ we usually write $\boldsymbol{x} \approx \mathcal{U} \boldsymbol{y}$ or just $\boldsymbol{x} \approx \boldsymbol{y}$ for $\boldsymbol{x}, \boldsymbol{y} \in{ }^{*} \mathbf{X}$.

An element $\boldsymbol{y} \in{ }^{*} \mathbf{X}$ is called nearstandard if $\boldsymbol{y} \approx \mathcal{U} \boldsymbol{x}$ for some $\boldsymbol{x} \in \mathbf{X}$; the set of all nearstandard elements of ${ }^{*} \mathbf{X}$ is denoted by $\mathrm{Ns}\left({ }^{*} \mathbf{X}\right)$. If the original uniform space $(\mathbf{X}, \mathcal{U})$ is Hausdorff (ie, if $\bigcap \mathcal{U}$ is the diagonal $\operatorname{Id}_{\mathbf{X}}$ ) then there is a well defined standard part mapping $\mathrm{Ns}\left({ }^{*} \mathbf{X}\right) \rightarrow \mathbf{X}$ sending every $\boldsymbol{y} \in \mathrm{Ns}\left({ }^{*} \mathbf{X}\right)$ to its standard part st $\boldsymbol{y}={ }^{\circ} \boldsymbol{y}$ which is the unique element $\boldsymbol{x} \in \mathbf{X}$ such that $\boldsymbol{y} \approx \boldsymbol{x}$. Then the original uniform space $(\mathbf{X}, \mathcal{U})$ can be reconstructed as the quotient $\mathbf{X} \cong \mathrm{Ns}^{*}\left({ }^{*}\right) / \approx$ (see the proof of Proposition 1.2.2).

In view of the works by Luxemburg [31], Henson [22] and Goldbring [15, 16] we accept as a fact that there is no canonical way how to define the finite elements in 
the nonstandard extension of a uniform space. Instead of looking for their adequate definition in terms of the uniform structure and standard elements we will treat the equivalence relation of infinitesimal nearness or indistinguishability arising from the uniformity on one hand, and the property of finiteness or accessibility on the other hand as almost independent or just loosely related phenomena to which there correspond different primitive concepts. Our basic nonstandard objects, by means of which we will study (sufficiently regular) topological spaces, will be ordered triples of the form $\left(X, E, X_{\mathrm{f}}\right)$ where $X$ is an internal set, $E$ is a monadic equivalence relation on $X$ and $X_{\mathrm{f}}$ is a galactic subset of $X$ which is $E$-closed, ie, $x \in X_{\mathrm{f}}$ and $(x, y) \in E$ imply $y \in X_{\mathrm{f}}$, for any $x, y \in X$. We will call them alternatively bounded monadic spaces or IMG spaces like in Ziman and Zlatoš [47], or IMG triplets, indicating that we do not consider the present terminology as definitive.

Intuitively, $X$ is viewed as the underlying or ambient set of the triplet, $E$ is the relation of indistinguishability or infinitesimal nearness on $X$, and $X_{\mathrm{f}}$ is the set of elements of $X$ encompassed by some observational horizon. The elements of $X_{\mathrm{f}}$ will be briefly referred to as the finite or accessible ones. To stress the role of the equivalence $E$ we will preferably write $x \approx_{E} y$ or just $x \approx y$ instead of $(x, y) \in E$, for $x, y \in X$, and call the set

$$
E[x]=\{y \in X: y \approx x\}
$$

of points indistinguishable from the point $x \in X$ the E-monad or just the monad of $x$. The $E$-closedness of $X_{\mathrm{f}}$ can be now expressed as the condition $E[x] \subseteq X_{\mathrm{f}}$ for any $x \in X_{\mathrm{f}}$. The quotient

$$
X_{\mathrm{f}} / E=X_{\mathrm{f}} / \approx=\left\{E[x]: x \in X_{\mathrm{f}}\right\}
$$

is called the observable trace of the triplet $\left(X, E, X_{\mathrm{f}}\right)$.

The restricted quotient mapping $X_{\mathrm{f}} \rightarrow X_{\mathrm{f}} / E$ reminds of the standard part mapping $\mathrm{Ns}\left({ }^{*} \mathbf{X}\right) \rightarrow \mathbf{X}$ in nonstandard extensions of Hausdorff uniform spaces. In order to underline this analogy (especially when viewing the monads as individual points and forgetting about their "sethood") we introduce the notation $E[x]=x^{b}$ for the monad of $x \in X$, and

$$
A^{b}=\left\{a^{b}: a \in A \cap X_{\mathrm{f}}\right\}
$$

for the observable trace of any set $A \subseteq X$. In particular, the observable trace $X^{b}=X_{\mathrm{f}}^{b}=X_{\mathrm{f}} / E$ of the triplet $\left(X, E, X_{\mathrm{f}}\right)$ should not be confused with the full quotient $X / E \supseteq X^{b}$. Conversely, for any $\mathbf{Y} \subseteq X^{b}$, we call the following set the pretrace of $\mathbf{Y}$ :

$$
\mathbf{Y}^{\sharp}=\left\{x \in X_{\mathrm{f}}: x^{b} \in \mathbf{Y}\right\}
$$

Given an IMG triplet $\left(X, E, X_{\mathrm{f}}\right)$, it is an easy exercise in saturation to show that for each internal relation $R \supseteq E$ on $X$ there is a symmetric internal relation $S \supseteq E$ on $X$ such 
that $S \circ S \subseteq R$. Similarly, for any internal set $A \subseteq X_{\mathrm{f}}$ there is an internal set $B \subseteq X_{\mathrm{f}}$ and a symmetric internal relation $S \supseteq E$ on $X$ such that $S[A] \subseteq B$. It follows that there is a downward directed system $\mathcal{R}$ of reflexive and symmetric internal relations on $X$, and an upward directed system $\mathcal{B}$ of internal subsets of $X$, both of admissible size, satisfying the following conditions:

$$
\begin{array}{ccc}
(\forall R \in \mathcal{R})(\exists S \in \mathcal{R})(S \circ S \subseteq R) & \text { and } & E=\bigcap \mathcal{R} \\
(\forall A \in \mathcal{B})(\exists B \in \mathcal{B})(\exists S \in \mathcal{R})(S[A] \subseteq B) & \text { and } & X_{\mathrm{f}}=\bigcup \mathcal{B}
\end{array}
$$

Then $\mathcal{R}$ becomes a base of a uniformity $\mathcal{U}_{E}$ on $X$ (non-Hausdorff, unless $E=\operatorname{Id}_{X}$ ). Another base for this uniformity (though not necessarily of admissible size) is formed by all the internal relations $R$ on $X$ such that $E \subseteq R$. A set $Y \subseteq X$ is open in the induced topology if and only if, for each $y \in Y$, there is an internal set $A$ such that $E[y] \subseteq A \subseteq Y$. In particular, $X_{\mathrm{f}}$ is an open subset of $X$. The closure of any set $Y \subseteq X$ is $\bigcap_{R \in \mathcal{R}} R[Y]$; for a monadic (in particular for an internal) $Y$ this is equal to $E[Y]$.

The observable traces

$$
R^{b}=\left\{\left(x^{b}, y^{b}\right):(x, y) \in R \cap\left(X_{\mathrm{f}} \times X_{\mathrm{f}}\right)\right\}
$$

of internal relations $R \in \mathcal{U}_{E}$ (or just $R \in \mathcal{R}$ ) form a uniformity base on the observable trace $X^{b}=X_{\mathrm{f}} / E$, inducing a Hausdorff completely regular topology on it.

We are particularly interested in representing Hausdorff locally compact spaces as observable traces of IMG triplets $\left(X, E, X_{\mathrm{f}}\right)$ with a hyperfinite ambient set $X$. To this end we introduce some types of indices of internal sets $A \subseteq X$ with respect to reflexive and symmetric internal relations $S \subseteq X \times X$ :

(a) The covering index or entropy of $A$ with respect to $S$, denoted by $\lfloor A: S\rfloor$, is the least $n \in{ }^{*} \mathbb{N}$, such that $A \subseteq S[F]$ for some hyperfinite sets $F \subseteq X$ with $n$ elements, or the symbol $\infty$ if there is no such $n$.

(b) The inner covering index of $A$ with respect to $S$, denoted by $\lfloor A: S\rfloor_{\mathrm{i}}$, is the least $n \in{ }^{*} \mathbb{N}$, such that $A \subseteq S[F]$ for some hyperfinite sets $F \subseteq A$ with $n$ elements, or the symbol $\infty$ if there is no such $n$.

(c) The independence index or the capacity of $A$ with respect to $S$ denoted by $\lceil A: S\rceil$, is the biggest $n \in{ }^{*} \mathbb{N}$ such that there is an $n$-element set $F \subseteq A$ satisfying $(x, y) \notin S$ for any distinct $x, y \in F$, or the symbol $\infty$ if there is no biggest $n$ with that property.

Then we have the following obvious inequalities (cf Roe [40]):

$$
\lceil A:(S \circ S)\rceil \leq\lfloor A: S\rfloor \leq\lfloor A: S\rfloor_{\mathrm{i}} \leq\lceil A: S\rceil
$$


If $G$ is an internal group then, instead of the symmetric and reflexive internal relation $S$ on $G$, we can take a symmetric internal subset $S \subseteq G$ containing the unit element $1 \in G$. The obvious modification of the above definitions and the last inequalities to this situation is left to the reader.

In the following proposition the expression $[A: S]$ denotes any of the indices $\lfloor A: S\rfloor$, $\lfloor A: S\rfloor_{\mathrm{i}}$ or $\lceil A: S\rceil$. The above inequalities guarantee that the formulations of (ii) with any particular choice for $[A: S]$ are all equivalent.

Proposition 1.2.1 Let $\left(X, E, X_{\mathrm{f}}\right)$ be a bounded monadic space. Then the following conditions are equivalent:

(i) All the internal subsets of $X_{\mathrm{f}}$ are relatively compact.

(ii) For any internal set $A \subseteq X_{\mathrm{f}}$ and every symmetric internal relation $S \supseteq E$ on $X$ the index $[A: S]$ is finite.

(iii) There is an external set $P \subseteq X_{\mathrm{f}}$ of admissible size such that $x \not z y$ for any distinct $x, y \in P$, and $X_{\mathrm{f}} \subseteq S[P]$ for every internal relation $S \supseteq E$.

(iv) For each $n \in{ }^{*} \mathbb{N}_{\infty}$ there is a hyperfinite set $H \subseteq X$ with at most $n$ elements such that $X_{\mathrm{f}} \subseteq E[H]$.

(v) For every infinite hyperfinite set $H \subseteq X_{\mathrm{f}}$ there are at least two distinct elements $x, y \in H$ such that $x \approx y$.

Proof (i) $\Leftrightarrow$ (ii) is plain, especially for the index $\lfloor A: S\rfloor_{\mathrm{i}}$.

(ii) $\Rightarrow$ (iii) Let $\mathcal{R}$ and $\mathcal{B}$ be systems of admissible size consisting of internal symmetric relations $R \subseteq X \times X$ and internal sets $B \subseteq X$, respectively, such that $E=\bigcap \mathcal{R}$ and $X_{\mathrm{f}}=\bigcup \mathcal{B}$. Condition (ii) with the index $\lfloor A: S\rfloor_{\mathrm{i}}$ implies that there is a system of finite sets $\left\{F_{R B}: R \in \mathcal{R}, B \in \mathcal{B}\right\}$ such that

$$
F_{R B} \subseteq B \subseteq R\left[F_{R B}\right]
$$

for all $R \in \mathcal{R}, B \in \mathcal{B}$. Then the set $Q=\bigcup_{R, B} F_{R B} \subseteq X_{\mathrm{f}}$ is of admissible size and, obviously, $X_{\mathrm{f}} \subseteq S[Q]$ for any internal relation $S \supseteq E$ on $X$. Now, it suffices to take for $P$ any subset of $Q$ containing exactly one point from every $E$-monad intersecting $Q$.

(iii) $\Rightarrow$ (iv) Let $P$ be the set guaranteed by (ii) and $n$ be an arbitrary infinite hypernatural number. Then, for each finite set $F \subseteq P$, we have $|F| \leq n$. By saturation, there is a hyperfinite set $H$ such that $P \subseteq H \subseteq X$ and $|H| \leq n$. It can be easily verified that $X_{\mathrm{f}} \subseteq E[H]$.

(iv) $\Rightarrow$ (v) Let $H \subseteq X_{\mathrm{f}}$ be any infinite hyperfinite set and $n$ be an infinite hypernatural number such that $n<|H|$. By (iv) there is a hyperfinite set $J \subseteq X$ such that $|J| \leq n$ and 
$X_{\mathrm{f}} \subseteq E[J]$. In particular, $H \subseteq E[J]$. As $|J|<|H|$, there is at least one element $z \in J$ such that the set $H \cap E[z]$ contains at least two distinct elements $x, y$. Then $x \approx y$.

(v) $\Rightarrow$ (ii) Assume that the independence index $\lceil A: S\rceil$ is not finite for some internal set $A \subseteq X_{\mathrm{f}}$ and an internal symmetric relation $S \supseteq E$. Then, by saturation, there is an infinite hyperfinite set $H \subseteq A$ such that $x \not z y$ for any distinct $x, y \in H$, contradicting (v).

Remark Let us turn the reader's attention to the following two details. First, in order to prove the implication (iii) $\Rightarrow$ (iv) the set $P$ just has to be of admissible size and satisfy the condition $X_{\mathrm{f}} \subseteq S[P]$ for every internal relation $S \supseteq E$; it is not necessary that it consists of pairwise distinguishable points. Second, (v) could be strengthened to the following Ramsey type statement: every infinite hyperfinite set $H \subseteq X_{\mathrm{f}}$ contains an infinite hyperfinite subset $H_{0}$ such that $x \approx y$ for any $x, y \in H_{0}$. Then the implication (iv) $\Rightarrow$ (v) could still be proved by picking an $n \in{ }^{*} \mathbb{N}_{\infty}$ such that, say, $n^{2} \leq|H|$.

The last condition (v) suggests calling the IMG triplets satisfying any (hence all) of the above conditions condensing (cf Mlček and Zlatoš [34, 35] as well as Ziman and Zlatoš [47]). Obviously, the observable trace of any condensing IMG space is locally compact, and the compact subsets of $X^{b}$ are exactly the observable traces $A^{b}$ of internal subsets $A \subseteq X_{\mathrm{f}}$. However, it should be kept in mind that this is a considerably stronger condition than just the local compactness of $X^{b}$. Nevertheless, we still have the following representation theorem.

Proposition 1.2.2 Let $\mathbf{X}$ be a Hausdorff locally compact topological space. Then, in every sufficiently saturated nonstandard universe, there is a condensing IMG triplet $\left(X, E, X_{\mathrm{f}}\right)$ such that $\mathbf{X}$ is homeomorphic to the observable trace $X^{b}$. If desirable, one can additionally arrange that the ambient space $X$ be hyperfinite.

Proof Let $\mathcal{U}$ be some uniformity inducing the topology of $\mathbf{X}$ and $\kappa$ be an uncountable cardinal bigger than the minimal cardinality of some base of $\mathcal{U}$ as well as of some open cover of $\mathbf{X}$ by relatively compact sets. Let us embed $\mathbf{X}$ into its nonstandard extension ${ }^{*} \mathbf{X}$ in some $\kappa$-saturated nonstandard universe and denote $\mathbf{E}_{\mathcal{U}}=\bigcap_{\mathbf{U} \in \mathcal{U}}{ }^{*} \mathbf{U}$. It can be easily verified that $\left({ }^{*} \mathbf{X}, \mathbf{E}_{\mathcal{U}}, \mathrm{Ns}^{*}\left({ }^{*} \mathbf{X}\right)\right)$ is a condensing IMG space whose observable trace (nonstandard hull) $\mathrm{Ns}\left({ }^{*} \mathbf{X}\right) / \mathbf{E}_{\mathcal{U}}$ is homeomorphic to $\mathbf{X}$.

Let $n$ be an arbitrary infinite hypernatural number and $X=H \subseteq{ }^{*} \mathbf{X}$ be the $n$-element hyperfinite set guaranteed by (iv) of 1.2.1. Now, it suffices to put $X_{\mathrm{f}}=\mathrm{Ns}\left({ }^{*} \mathbf{X}\right) \cap X$, $E=\mathbf{E}_{\mathcal{U}} \cap(X \times X)$, and we get another condensing IMG triplet $\left(X, E, X_{\mathrm{f}}\right)$ with the observable trace $X_{\mathrm{f}} / E \cong \mathrm{Ns}\left({ }^{*} \mathbf{X}\right) / \mathbf{E}_{\mathcal{U}} \cong \mathbf{X}$ and hyperfinite ambient space $X$. 
The crucial property of the internal inclusion mapping $\operatorname{Id}_{X}: X \rightarrow{ }^{*} \mathbf{X}$ (under the identification ${ }^{*} \boldsymbol{x}=\boldsymbol{x}$ for $\boldsymbol{x} \in \mathbf{X}$ ) is namely that for each $\boldsymbol{x} \in \mathbf{X}$ there is some $x \in X$ such that $x \approx \boldsymbol{x}$. More generally, given any hyperfinite set $X$, an internal mapping $\eta: X \rightarrow{ }^{*} \mathbf{X}$ is called a hyperfinite infinitesimal approximation, briefly an HFI approximation, of the Hausdorff uniform space $\mathbf{X}$ if:

$$
(\forall \boldsymbol{x} \in \mathbf{X})(\exists x \in X)(\eta(x) \approx \boldsymbol{x})
$$

It is called an injective HFI approximation if, additionally, $\eta$ is an injective mapping.

The standard counterpart of this notion can be formulated in terms of finite approximations and rasters. A raster in a uniform space $(\mathbf{X}, \mathcal{U})$, briefly an $\mathbf{X}$-raster, is a pair $(\mathbf{K}, \mathbf{U})$ consisting of a compact set $\mathbf{K} \subseteq \mathbf{X}$ and a symmetric entourage $\mathbf{U} \in \mathcal{U}$, such that $\mathbf{U}[\boldsymbol{x}] \subseteq \mathbf{K}$ for some $\boldsymbol{x} \in \mathbf{K}$. Given an $\mathbf{X}$-raster $(\mathbf{K}, \mathbf{U})$, a mapping $\eta: X \rightarrow \mathbf{X}$ is called a finite $(\mathbf{K}, \mathbf{U})$ approximation or just a $(\mathbf{K}, \mathbf{U})$ approximation of $\mathbf{X}$ if:

$$
(\forall \boldsymbol{x} \in \mathbf{K})(\exists x \in X)((\eta(x), \boldsymbol{x}) \in \mathbf{U})
$$

If the map $\eta$ is injective then it is called an injective $(\mathbf{K}, \mathbf{U})$ approximation.

A partially ordered set, briefly a poset $(I, \leq)$, is a set $I$ with a reflexive, antisymmetric and transitive binary relation $\leq$; if the relation $\leq$ is upward directed then $(I, \leq)$ is called a directed poset. Obviously, the set of all $\mathbf{X}$-rasters in a uniform space $(\mathbf{X}, \mathcal{U})$, partially ordered by the relation

$$
(\mathbf{K}, \mathbf{U}) \leq(\mathbf{Q}, \mathbf{V}) \Leftrightarrow \mathbf{K} \subseteq \mathbf{Q} \text { and } \mathbf{V} \subseteq \mathbf{U}
$$

is a directed poset.

Let $(\mathbf{X}, \mathcal{U})$ be a Hausdorff uniform space and $(I, \leq)$ be a directed poset. Then a system of mappings $\left(\eta_{i}: X_{i} \rightarrow \mathbf{X}\right)_{i \in I}$ is called an approximating system of the space $(\mathbf{X}, \mathcal{U})$ provided each $X_{i}$ is a finite set and, for any $\mathbf{X}$-raster $(\mathbf{K}, \mathbf{U})$, there is $i \in I$ such that $\eta_{j}: X_{j} \rightarrow \mathbf{X}$ is a $(\mathbf{K}, \mathbf{U})$ approximation of $\mathbf{X}$ for each $j \in I, j \geq i$.

It can be easily seen that every Hausdorff locally compact uniform space $(\mathbf{X}, \mathcal{U})$ has some approximating system $\left(\eta_{i}: X_{i} \rightarrow \mathbf{X}\right)_{i \in I}$ such that each $X_{i}$ is a finite subset of $\mathbf{X}$ and $\eta_{i}: X_{i} \rightarrow \mathbf{X}$ is the inclusion mapping. Assuming $\kappa$-saturation for some sufficiently big $\kappa$, we can find an ${ }^{*} \mathbf{X}$-raster $\left(\mathbf{K}^{\prime}, \mathbf{U}^{\prime}\right)$, formed by a ${ }^{*}$ compact set $\mathbf{K}^{\prime} \subseteq{ }^{*} \mathbf{X}$ and an entourage $\mathbf{U}^{\prime} \in{ }^{*} \mathcal{U}$, such that $(\mathbf{K}, \mathbf{U}) \leq\left(\mathbf{K}^{\prime}, \mathbf{U}^{\prime}\right)$ for all $\mathbf{X}$-rasters $(\mathbf{K}, \mathbf{U})$. Then there is $i \in{ }^{*} I$ such that the hyperfinite set $X_{i} \subseteq{ }^{*} \mathbf{X}$ (together with the inclusion map $\left.\eta_{i}: X_{i} \rightarrow{ }^{*} \mathbf{X}\right)$ is an internal $\left(\mathbf{K}^{\prime}, \mathbf{U}^{\prime}\right)$ approximation of ${ }^{*} \mathbf{X}$, hence an HFI approximation of $\mathbf{X}$. Putting $X=X_{i}, E=\mathbf{E}_{\mathcal{U}} \cap(X \times X)$, and $\left.X_{\mathrm{f}}=\mathrm{Ns}^{*} \mathbf{X}\right) \cap X$, we obtain a condensing IMG triplet $\left(X, E, X_{\mathrm{f}}\right)$ with a hyperfinite ambient space $X$ and observable trace $X^{b}=X_{\mathrm{f}} / E \cong \mathbf{X}$. This gives another proof of Proposition 1.2.2. 
Given two IMG spaces $\left(X, E, X_{\mathrm{f}}\right),\left(Y, F, Y_{\mathrm{f}}\right)$, an internal mapping $f: D \rightarrow Y$ is called a triplet morphism if $X_{\mathrm{f}} \subseteq D \subseteq X$, it preserves finiteness, ie $f(x) \in Y_{\mathrm{f}}$ for all $x \in X_{\mathrm{f}}$, and it is $S$-continuous on $X_{\mathrm{f}}$, ie

$$
x \approx_{E} y \Rightarrow f(x) \approx_{F} f(y)
$$

whenever $x, y \in X_{\mathrm{f}}$. In such a case we write $f:\left(X, E, X_{\mathrm{f}}\right) \rightarrow\left(Y, F, Y_{\mathrm{f}}\right)$. Note that every triplet morphism $f$ with domain $D$ can be formally extended to an everywhere defined triplet morphism $\widetilde{f}: X \rightarrow Y$ in an arbitrary way.

Every triplet morphism $f:\left(X, E, X_{\mathrm{f}}\right) \rightarrow\left(Y, F, Y_{\mathrm{f}}\right)$ induces a continuous mapping $f^{b}: X^{b} \rightarrow Y^{b}$, called the observable trace of $f$, (correctly) defined by

$$
f^{b}\left(x^{b}\right)=f(x)^{b}
$$

for $x \in X_{\mathrm{f}}$. Two triplet morphisms $f, g:\left(X, E, X_{\mathrm{f}}\right) \rightarrow\left(Y, F, Y_{\mathrm{f}}\right)$ are called equivalent if they have the same observable trace $f^{b}=g^{b}$. However, we can put

$$
f \underset{\bar{X}_{\mathrm{f}}}{\approx} g \Leftrightarrow\left(\forall x \in X_{\mathrm{f}}\right)(f(x) \approx g(x))
$$

for any internal functions $f: D_{1} \rightarrow Y, g: D_{2} \rightarrow Y$ such that $X_{\mathrm{f}} \subseteq D_{1} \cap D_{2}$. This relation of infinitesimal nearness on finite elements is a monadic equivalence on any set $Y^{D}$ of all internal functions $D \rightarrow Y$, where $X_{\mathrm{f}} \subseteq D$. For two triplet morphisms $f, g:\left(X, E, X_{\mathrm{f}}\right) \rightarrow\left(Y, F, Y_{\mathrm{f}}\right)$ we have

$$
f \underset{\mathbb{X}_{\mathrm{f}}}{\approx} g \Leftrightarrow f^{b}=g^{b}
$$

indicating a fundamental role of this indistinguishability equivalence. Let us remark, without being precise, that for a condensing IMG space $\left(X, E, X_{\mathrm{f}}\right)$ the equivalence ${\widetilde{X_{\mathrm{f}}}}_{\mathrm{f}}$ corresponds to the compact-open topology on the space $\mathrm{C}\left(X^{b}, Y^{b}\right)$ of all continuous functions $X^{b} \rightarrow Y^{b}$.

Another important feature of condensing IMG spaces is the lifting property. Given a mapping $f: X^{b} \rightarrow Y^{b}$ between the observable traces, an internal mapping $f: D \rightarrow Y$ is called a lifting of $f$ if $X_{\mathrm{f}} \subseteq D \subseteq X$ and

$$
\boldsymbol{f}\left(x^{b}\right)=f(x)^{b}
$$

for each $x \in X_{\mathrm{f}}$. Notice that an internal mapping $f: D \rightarrow Y$, satisfying the last equality is necessarily $S$-continuous on $X_{\mathrm{f}}$ and satisfies $f\left[X_{\mathrm{f}}\right] \subseteq Y_{\mathrm{f}}$, hence, it is a triplet morphism $\left(X, E, X_{\mathrm{f}}\right) \rightarrow\left(Y, F, Y_{\mathrm{f}}\right)$. Conversely, a triplet morphism $f:\left(X, E, X_{\mathrm{f}}\right) \rightarrow\left(Y, F, Y_{\mathrm{f}}\right)$ is a lifting of $\boldsymbol{f}$ if and only if $\boldsymbol{f}=f^{b}$ is the observable trace of $f$. Then $\boldsymbol{f}$ necessarily is continuous, as well. Thus only continuous mappings between observable traces of IMG triplets have $S$-continuous liftings on $X_{\mathrm{f}}$. The point is that for a condensing IMG 
triplet $\left(X, E, X_{\mathrm{f}}\right)$ this necessary continuity condition is also sufficient for the existence of liftings.

Proposition 1.2.3 Let $\left(X, E, X_{\mathrm{f}}\right),\left(Y, F, Y_{\mathrm{f}}\right)$ be two IMG spaces. If $\left(X, E, X_{\mathrm{f}}\right)$ is condensing then a mapping $f: X^{b} \rightarrow Y^{b}$ is continuous if and only if $f$ has an internal $S$-continuous lifting on $X_{\mathrm{f}}$.

Sketch of proof Let's focus only on the nontrivial implication. Assume that $f$ is continuous and denote by

$$
\boldsymbol{f}^{\sharp}=\left\{(x, y) \in X \times Y: \boldsymbol{f}\left(x^{b}\right)=y^{b}\right\}
$$

its pretrace considered as a set $f^{\sharp} \subseteq X_{\mathrm{f}} \times Y_{\mathrm{f}}$ in the IMG space $\left(X \times Y, E \times F, X_{\mathrm{f}} \times Y_{\mathrm{f}}\right)$. It suffices to show that, for every internal set $D \subseteq X_{\mathrm{f}}$, the restriction $f^{\sharp}\lceil D$ is monadic. Then, by the Internal Choice Lemma 1.1.1, there is an internal choice function $f$ from $\boldsymbol{f}^{\sharp}$ on its domain $X_{\mathrm{f}}$. Obviously, this $f$ is an $S$-continuous lifting of $\boldsymbol{f}$.

At least, there is some (not necessarily internal) function $\varphi: X_{\mathrm{f}} \rightarrow Y$, such that $\boldsymbol{f}\left(x^{b}\right)=\varphi(x)^{b}$ for each $x \in X_{\mathrm{f}}$.

Let us fix some nonempty internal set $D \subseteq X_{\mathrm{f}}$. Let further $\mathcal{R}, \mathcal{S}$ be some systems of admissible size consisting of symmetric internal relations on $X, Y$ respectively, such that $E=\bigcap \mathcal{R}, F=\bigcap \mathcal{S}$, and $P \subseteq X_{\mathrm{f}}$ be a set of admissible size, such that $R[P]=X_{\mathrm{f}}$ for every $R \in \mathcal{R}$, whose existence is guaranteed in Proposition 1.2.1 (iii). Denote by $\mathcal{I}_{D}$ the set of all ordered triples $(S, R, A)$ such that $S \in \mathcal{S}, R \in \mathcal{R}, A$ is a finite subset of $P$ subject to $D \subseteq R[A]$, and

$$
\left(x^{b}, y^{b}\right) \in R^{b} \Rightarrow\left(\boldsymbol{f}\left(x^{b}\right), \boldsymbol{f}\left(y^{b}\right)\right) \in S^{b}
$$

for all $x, y \in D$. Obviously, $\mathcal{I}_{D}$ is of admissible size. Moreover, $D^{b} \subseteq X^{b}$ is compact, hence $f$ is uniformly continuous on $D^{b}$. Therefore, for any $S \in \mathcal{S}$ there is an $R \in \mathcal{R}$ and a finite $A \subseteq P$ such that $(S, R, A) \in \mathcal{I}_{D}$. For any triple $i=(S, R, A) \in \mathcal{I}_{D}$ we put:

$$
\Phi_{i}=\bigcup_{a \in A}(D \cap R[a]) \times S[\varphi(a)]
$$

As $A$ is finite, each $\Phi_{i}$ is an internal relation. Using the continuity of $f$, the equality

$$
f^{\sharp} \mid D=\bigcap_{i \in \mathcal{I}_{D}} \Phi_{i}
$$

can be checked in a routine way, resembling the standard proof of the Closed Graph Theorem for a continuous function into a Hausdorff space. 
Corollary 1.2.4 Let $\left(X, E, X_{\mathrm{f}}\right),\left(Y, F, Y_{\mathrm{f}}\right)$ be condensing bounded monadic spaces with homeomorphic observable traces $X^{b} \cong Y^{b}$. Then there exist triplet morphisms $f:\left(X, E, X_{\mathrm{f}}\right) \rightarrow\left(Y, F, Y_{\mathrm{f}}\right)$ and $g:\left(Y, F, Y_{\mathrm{f}}\right) \rightarrow\left(X, E, X_{\mathrm{f}}\right)$ such that

$$
g(f(x)) \approx x \quad \text { and } \quad f(g(y)) \approx y
$$

for all $x \in X_{\mathrm{f}}, y \in Y_{\mathrm{f}}$.

Naturally, a triplet morphism $f:\left(X, E, X_{\mathrm{f}}\right) \rightarrow\left(Y, F, Y_{\mathrm{f}}\right)$ to which there is a triplet morphism $g:\left(Y, F, Y_{\mathrm{f}}\right) \rightarrow\left(X, E, X_{\mathrm{f}}\right)$ satisfying the above condition will be called a triplet isomorphism. Since it is clear that, for any pair of triplet isomorphisms $f:\left(X, E, X_{\mathrm{f}}\right) \rightarrow\left(Y, F, Y_{\mathrm{f}}\right), g:\left(Y, F, Y_{\mathrm{f}}\right) \rightarrow\left(X, E, X_{\mathrm{f}}\right)$ satisfying the conditions of Corollary 1.2.4, their observable traces $f^{b}: X^{b} \rightarrow Y^{b}, g^{b}: Y^{b} \rightarrow X^{b}$ form a pair of mutually inverse homeomorphisms, the last corollary can be briefly restated as follows: Condensing IMG triplets are isomorphic if and only if they have homeomorphic observable traces.

Corollary 1.2.5 Let $\mathbf{X}$ be a Hausdorff locally compact uniform space and $\left(X, E, X_{\mathrm{f}}\right)$ be a condensing IMG space with a hyperfinite ambient set $X$ and the observable trace $X^{b}$ homeomorphic to $\mathbf{X}$. Then there is an HFI approximation $\eta: X \rightarrow{ }^{*} \mathbf{X}$ which is a triplet isomorphism $\eta:\left(X, E, X_{\mathrm{f}}\right) \rightarrow\left({ }^{*} \mathbf{X}, \mathbf{E}_{\mathcal{U}}, \mathrm{Ns}\left({ }^{*} \mathbf{X}\right)\right)$.

In the last part of this section we briefly recall the Loeb measure construction and the construction of regular Borel measures on Hausdorff locally compact spaces by pushing down Loeb measures.

Let $X \neq \emptyset$ be a hyperfinite set and $d: X \rightarrow{ }^{*} \mathbb{R}$ be an internal function such that $d(x) \geq 0$ for each $x \in X$. Intuitively, $d(x)$ is viewed as the "weight" of the point $x$. Then $d$ induces the internal (hyper)finitely additive measure $\nu_{d}: \mathcal{P}(X) \rightarrow{ }^{*} \mathbb{R}$ on the internal Boolean algebra $\mathcal{P}(X)$ of all internal subsets of $X$, given by $\nu_{d}(A)=\sum_{a \in A} d(a)$ for internal $A \subseteq X$. Putting

$$
\left({ }^{\circ} \nu_{d}\right)(A)= \begin{cases}{ }^{\circ}\left(\nu_{d}(A)\right) & \text { if } \nu_{d}(A) \text { is finite } \\ \infty & \text { otherwise }\end{cases}
$$

we get a finitely additive (non-negative) measure ${ }^{\circ} \nu_{d}: \mathcal{P}(X) \rightarrow \mathbb{R} \cup\{\infty\}$ which has a unique extension to a $\sigma$-additive measure $\lambda_{d}: \widetilde{\mathcal{P}}(X) \rightarrow \mathbb{R} \cup\{\infty\}$ defined on the $\sigma$-algebra $\widetilde{\mathcal{P}}(X)$ of all subsets of $X$ generated by all monadic (or, equivalently, by all galactic) subsets of $X$. (If $\kappa=\aleph_{1}$ then $\widetilde{\mathcal{P}}(X)$ is simply the $\sigma$-algebra generated by all internal subsets of $X$.) Then $\lambda_{d}$ is the Loeb measure induced by the internal function $d$ (cf Cutland [7], Landers and Rogge [27], Loeb [28], or Ross [41]). 
For the rest of this section, $\mathbf{X}$ denotes a Hausdorff locally compact space, represented as (and identified with) the observable trace $\mathbf{X} \cong X^{b}$ of a condensing IMG triplet $\left(X, E, X_{\mathrm{f}}\right)$ with a hyperfinite ambient set $X$ by means of an HFI approximation $\eta: X \rightarrow{ }^{*} \mathbf{X}$. Assume that the nonnegative internal function $d: X \rightarrow{ }^{*} \mathbb{R}$ satisfies additionally the condition that $\nu_{d}(A)$ is finite for each internal set $A \subseteq X_{\mathrm{f}}$. Then the set $X_{\mathrm{f}}$ belongs to the algebra $\widetilde{\mathcal{P}}(X)$ and the system

$$
\widetilde{\mathcal{P}}\left(X_{\mathrm{f}}\right)=\left\{A \in \widetilde{\mathcal{P}}(X): A \subseteq X_{\mathrm{f}}\right\}
$$

is again a $\sigma$-algebra of subsets of $X_{\mathrm{f}}$. Moreover, the pretrace $\mathbf{Y}^{\sharp}$ of every Borel set $\mathbf{Y} \subseteq \mathbf{X}$ belongs to $\widetilde{\mathcal{P}}\left(X_{\mathrm{f}}\right)$, hence the observable trace map ${ }^{b}: X_{\mathrm{f}} \rightarrow \mathbf{X}=X^{b}$ is measurable. Pushing down the Loeb measure along this map, ie putting

$$
\boldsymbol{m}_{d}(\mathbf{Y})=\lambda_{d}\left(\mathbf{Y}^{\sharp}\right)
$$

for Borel $\mathbf{Y} \subseteq \mathbf{X}$, we obtain a regular Borel measure $\boldsymbol{m}_{d}$ on $\mathbf{X}$ (which, if desirable, can be extended to a complete measure by the Carathéodory construction). The converse is essentially due to Anderson [3] who proved it in case $\boldsymbol{m}$ is a probability measure on $\mathbf{X}$, which directly extends to the case that $\boldsymbol{m}(\mathbf{X})$ is finite. In the next proposition we manage without this assumption.

Proposition 1.2.6 Every nonnegative regular Borel measure $\boldsymbol{m}$ on $\mathbf{X}$ has the form $\boldsymbol{m}=\boldsymbol{m}_{d}$ for some internal function $d: X \rightarrow{ }^{*} \mathbb{R}$, such that $d(x) \geq 0$ for each $x \in X$, and $\nu_{d}(A)$ is finite for each internal set $A \subseteq X_{\mathrm{f}}$. Additionally, we can arrange that $d(x)>0$ for each $x \in X$.

Sketch of proof There is a ${ }^{*} \mathbf{X}$-raster $(\mathbf{K}, \mathbf{U})$ where $\mathbf{U} \in{ }^{*} \mathcal{U}$ is ${ }^{*}$ open in ${ }^{*} \mathbf{X} \times{ }^{*} \mathbf{X}$ and $\mathbf{K} \subseteq{ }^{*} \mathbf{X}$ is a ${ }^{*}$ compact set, such that $\mathbf{U} \subseteq \mathbf{E}_{\mathcal{U}}, \operatorname{Ns}\left({ }^{*} \mathbf{X}\right) \subseteq \mathbf{K}$, and $\eta$ is a $(\mathbf{K}, \mathbf{U})$ approximation of ${ }^{*} \mathbf{X}$. Since $\eta$ has a hyperfinite range, there is an internal mapping $\sigma:{ }^{*} \mathbf{X} \rightarrow X$ such that $(\eta \circ \sigma \circ \eta)(x)=\eta(x)$ and $((\eta \circ \sigma)(\boldsymbol{x}), \boldsymbol{x}) \in \mathbf{U}$ for $x \in X, \boldsymbol{x} \in \mathbf{K}$, and each of the sets $\{\boldsymbol{x} \in \mathbf{K}:(\eta \circ \sigma)(\boldsymbol{x})=\eta(x)\}$ is ${ }^{*}$ Borel in ${ }^{*} \mathbf{X}$. We put

$$
d(x)= \begin{cases}\frac{{ }^{*}(\{\boldsymbol{x} \in \mathbf{K}:(\eta \circ \sigma)(\boldsymbol{x})=\eta(x)\})}{|\{y \in X: \eta(y)=\eta(x)\}|} & \text { if } \eta(x) \in \mathbf{U}[\mathbf{K}] \\ 0 & \text { if } \eta(x) \notin \mathbf{U}[\mathbf{K}]\end{cases}
$$

for $x \in X .{ }^{1}$ The verification that the internal mapping $d$ has all the required properties is left to the reader. Replacing each value $d(x)$ by $d(x)+\varepsilon$, where $\varepsilon$ is a positive infinitesimal such that $\varepsilon|X| \approx 0$, we can satisfy also the additional requirement.

\footnotetext{
${ }^{1}$ In fact, the values of $d(x)$ for $\eta(x) \notin \mathbf{U}[\mathbf{K}]$ can be chosen arbitrarily without affecting the resulting measure $\boldsymbol{m}_{d}$.
} 


\subsection{Bounded monadic groups}

A bounded monadic group is an ordered triple $\left(G, G_{0}, G_{\mathrm{f}}\right)$ consisting of an internal group $G$ (which means that $G$ is an internal set, endowed with internal operations of group multiplication and taking inverses), a monadic subgroup $G_{0} \subseteq G$ and a galactic subgroup $G_{\mathrm{f}} \subseteq G$, such that $G_{0} \triangleleft G_{\mathrm{f}}$ (ie, $G_{0}$ is a normal subgroup of $G_{\mathrm{f}}$ ). Intuitively, $G_{0}$ is viewed as the subgroup of infinitesimals and $G_{\mathrm{f}}$ is viewed as the subgroup of finite elements in $G$. Bounded monadic groups will be alternatively referred to as $I M G$ group triplets (cf Ziman and Zlatoš [47]).

Every IMG group triplet gives rise to two IMG spaces: $\left(G, E_{l}, G_{\mathrm{f}}\right)$ and $\left(G, E_{r}, G_{\mathrm{f}}\right)$, where $E_{l}, E_{r}$ denote the left and the right equivalence relation on $G$ corresponding to $G_{0}$, respectively. Though they may differ and induce different uniformities on the ambient group $G$, they induce the same uniformity on $G_{\mathrm{f}}$; in other words, the bounded monadic spaces $\left(G, E_{l}, G_{\mathrm{f}}\right),\left(G, E_{r}, G_{\mathrm{f}}\right)$ are isomorphic via the identity mapping $\operatorname{Id}_{G}: G \rightarrow G$. The reason is that the left and the right uniformities on $G_{\mathrm{f}}$ are uniquely determined by the equivalence relations $E_{l} \cap\left(G_{\mathrm{f}} \times G_{\mathrm{f}}\right)$ and $E_{r} \cap\left(G_{\mathrm{f}} \times G_{\mathrm{f}}\right)$ on $G_{\mathrm{f}}$, respectively, which coincide because of the normality condition $G_{0} \triangleleft G_{\mathrm{f}}$.

The group $G_{\mathrm{f}}$, as well as the observable trace $G^{b}=G_{\mathrm{f}} / G_{0}$, endowed with the topologies induced by the subgroup $G_{0}$, described in Section 1.2, become topological groups and the observable trace map $x \mapsto x^{b}$ is a continuous surjective homomorphism of topological groups $G_{\mathrm{f}} \rightarrow G^{b}$. (On the other hand, unless $G_{0} \triangleleft G$, the topology on $G$ induced by $G_{0}$ does not turn it into a topological group.)

Two paradigmatic examples of IMG group triplets arise from the nonstandard extensions ${ }^{*} \mathbb{R}$ and ${ }^{*} \mathbb{C}$ of the additive groups of the fields $\mathbb{R}$ and $\mathbb{C}$ of the real and complex numbers, respectively. We denote by $\mathbb{F}^{*} \mathbb{R} \subseteq{ }^{*} \mathbb{R}$ or $\mathbb{F}{ }^{*} \mathbb{C} \subseteq{ }^{*} \mathbb{C}$ the galactic subrings of finite (bounded) hyperreal or hypercomplex numbers, and by $\mathbb{I}^{*} \mathbb{R} \subseteq \mathbb{F}^{*} \mathbb{R}$ or $\mathbb{I}^{*} \mathbb{C} \subseteq \mathbb{F}^{*} \mathbb{C}$ the monadic ideals of infinitesimal hyperreal or hypercomplex numbers. Then $\left({ }^{*} \mathbb{R}, \mathbb{I}^{*} \mathbb{R}, \mathbb{F}^{*} \mathbb{R}\right)$ and $\left({ }^{*} \mathbb{C}, \mathbb{I}^{*} \mathbb{C}, \mathbb{F}^{*} \mathbb{C}\right)$ are $\mathrm{IMG}$ group triplets with observable traces $\mathbb{F}^{*} \mathbb{R} / \mathbb{I}^{*} \mathbb{R}$ and $\mathbb{F}^{*} \mathbb{C} / \mathbb{I}^{*} \mathbb{C}$, isomorphic to the standard structures $\mathbb{R}$ and $\mathbb{C}$, respectively, even as topological fields. For $x \in{ }^{*} \mathbb{R}, x \geq 0$, we sometimes write $x<\infty$ instead of $x \in \mathbb{F}^{*} \mathbb{R}$, and $x \sim \infty$ instead of $x \in{ }^{*} \mathbb{R} \backslash \mathbb{F}^{*} \mathbb{R}$.

In any IMG group triplet $\left(G, G_{0}, G_{\mathrm{f}}\right)$, the systems $\mathcal{R}$ and $\mathcal{B}$ from Section 1.2 can be replaced by a single system $\mathcal{Q}$ of admissible size, directed both upward and downward, consisting of symmetric internal subsets of $G$, such that:

$$
(\forall Q \in \mathcal{Q})(\exists R, S \in \mathcal{Q})(R \cdot R \subseteq Q \& Q \cdot Q \subseteq S)
$$




$$
\begin{gathered}
(\forall Q, R \in \mathcal{Q})(\exists S \in \mathcal{Q})\left(\bigcup_{x \in R} x S x^{-1} \subseteq Q\right) \\
G_{0}=\bigcap \mathcal{Q} \quad G_{\mathrm{f}}=\bigcup \mathcal{Q}
\end{gathered}
$$

If $G_{0}$ is the intersection and $G_{\mathrm{f}}$ is the union of countably many internal sets then we can assume that $\mathcal{Q}=\left\{Q_{n}: n \in \mathbb{Z}\right\}$ and the symmetric internal sets $Q_{n} \subseteq G$ satisfy:

$$
\begin{gathered}
(\forall n \in \mathbb{Z})\left(Q_{n} \cdot Q_{n} \subseteq Q_{n+1}\right) \\
(\forall n \in \mathbb{N})\left(\bigcup_{x \in Q_{n}} x Q_{-n-1} x^{-1} \subseteq Q_{-n}\right) \\
G_{0}=\bigcap_{n \in \mathbb{Z}} Q_{n}=\bigcap_{n \in \mathbb{N}} Q_{-n} \quad G_{\mathrm{f}}=\bigcup_{n \in \mathbb{Z}} Q_{n}=\bigcup_{n \in \mathbb{N}} Q_{n}
\end{gathered}
$$

An internal function $\varrho: G \rightarrow{ }^{*} \mathbb{R}$ is called an internal gauge or just a gauge on the internal group $G$ if

$$
\begin{gathered}
\varrho(x)=0 \Leftrightarrow x=1 \\
\varrho(x)=\varrho\left(x^{-1}\right) \\
\varrho(x y) \leq \varrho(x)+\varrho(y)
\end{gathered}
$$

for all $x, y, \in G(\varrho(x) \geq 0$ already follows). Obviously, if $\varrho$ is a gauge on $G$ then $\varrho\left(x^{-1} y\right)$ is a left invariant and $\varrho\left(x y^{-1}\right)$ is a right invariant metric on $G$. Conversely, if $d: G \times G \rightarrow G$ is an internal metric on $G$ such that $d\left(x^{-1}, y^{-1}\right)=d(x, y)$ for all $x, y \in G$ then $\varrho(x)=d(x, 1)$ is a gauge on $G$ and $d$ is left (right) invariant if and only if $d(x, y)=\varrho\left(x^{-1} y\right)\left(d(x, y)=\varrho\left(x y^{-1}\right)\right)$.

The set of all gauges on $G$ is partially ordered by the relation

$$
\varrho \leq \sigma \Leftrightarrow(\forall x \in G)(\varrho(x) \leq \sigma(x))
$$

A set $\mathcal{V}$ of gauges on $G$ is called bidirected if it is both downward and upward directed with respect to the above partial order $\leq$.

Using a slightly modified Birkhoff-Kakutani style argument, one can prove the following version of the metrization theorem for bounded monadic group triplets.

Proposition 1.3.1 Let $\left(G, G_{0}, G_{\mathrm{f}}\right)$ be an IMG group triplet. If $G_{0}$ is an intersection and $G_{\mathrm{f}}$ is a union of countably many internal sets, then there is a gauge $\varrho: G \rightarrow{ }^{*} \mathbb{R}$ such that:

$$
\begin{aligned}
& G_{0}=\{x \in G: \varrho(x) \approx 0\} \\
& G_{\mathrm{f}}=\{x \in G: \varrho(x)<\infty\}
\end{aligned}
$$


In general, there is a bidirected set $\mathcal{V}$ of admissible size of gauges on $G$, such that:

$$
\begin{aligned}
& G_{0}=\{x \in G:(\forall \varrho \in \mathcal{V})(\varrho(x) \approx 0)\} \\
& G_{\mathrm{f}}=\{x \in G:(\exists \varrho \in \mathcal{V})(\varrho(x)<\infty)\}
\end{aligned}
$$

Sketch of proof In the countable case we give just the formula for $\varrho$; the fact that it has all the required properties can be verified in a routine way.

There is a sequence $\left(A_{n}\right)_{n \in \mathbb{Z}}$ of symmetric internal subsets of $G$ such that

$$
G_{0}=\bigcap_{n \in \mathbb{Z}} A_{n} \quad G_{\mathrm{f}}=\bigcup_{n \in \mathbb{Z}} A_{n}
$$

and

$$
A_{n} \cdot A_{n} \cdot A_{n} \subseteq A_{n+1}
$$

for each $n \in \mathbb{Z}$. By saturation, it can be extended to an internal sequence $\left(A_{n}\right)_{-m \leq n \leq m}$ for some $m \in{ }^{*} \mathbb{N}_{\infty}$, such that $A_{-m}=\{0\}, A_{m}=G$, and the above inclusions hold for all $n$ such that $-m \leq n<m$. Let us put

$$
\mu(x)=\min \left\{n: \quad-m \leq n \leq m \& x \in A_{n}\right\}
$$

and denote by

$$
\mathbf{P}(G)=\bigcup_{n \in * \mathbb{N}} G^{n}
$$

the internal set of all hyperfinite internal progressions in $G$. For any progression $\mathbf{x}=\left(x_{1}, \ldots, x_{n}\right) \in \mathbf{P}(G)$ we denote by $|\mathbf{x}|=n$ its length and put:

$$
\Pi(\mathbf{x})=\prod_{i=1}^{n} x_{i} \quad \text { and } \quad w(\mathbf{x})=\sum_{i=1}^{n} 2^{\mu\left(x_{i}\right)}
$$

For the empty progression ()$=\emptyset$ (ie, in case $n=0$ ) this means that $\Pi(\emptyset)=1$ and $w(\emptyset)=0$. Then, finally,

$$
\varrho(x)={ }^{*} \inf \{w(\mathbf{x}): \mathbf{x} \in \mathbf{P}(G) \& \Pi(\mathbf{x})=x\}
$$

is the desired internal gauge.

In the general case, let us invoke the system $\mathcal{Q}$ introduced in the beginning of this section. For each $S \in \mathcal{Q}$ there is a sequence $\left(A_{n}^{S}\right)_{n \in \mathbb{Z}}$ of sets from $\mathcal{Q}$ such that $A_{0}^{S}=S$,

$$
G_{0} \subseteq \bigcap_{n \in \mathbb{Z}} A_{n}^{S} \quad \bigcup_{n \in \mathbb{Z}} A_{n}^{S} \subseteq G_{\mathrm{f}}
$$

and

$$
A_{n}^{S} \cdot A_{n}^{S} \cdot A_{n}^{S} \subseteq A_{n+1}^{S}
$$


for each $n$. Let $\varrho^{S}$ be the gauge constructed from (some hyperfinite extension of) this sequence. Let us denote:

$$
\mathcal{V}_{0}=\left\{\varrho^{S}: S \in \mathcal{Q}\right\}
$$

Then $\mathcal{V}_{0}$ obviously has all the properties required for $\mathcal{V}$, except perhaps for the bidirectedness. To fix this issue, we define

$$
\begin{aligned}
& \left(\varrho_{1} \wedge \varrho_{2}\right)(x)={ }^{*} \inf \left\{\sum_{i=1}^{|\mathbf{x}|} \min \left\{\varrho_{1}\left(x_{i}\right), \varrho_{2}\left(x_{i}\right)\right\}: \mathbf{x} \in \mathbf{P}(G) \& \Pi(\mathbf{x})=x\right\} \\
& \left(\varrho_{1} \vee \varrho_{2}\right)(x)=\max \left\{\varrho_{1}(x), \varrho_{2}(x)\right\}
\end{aligned}
$$

for any gauges $\varrho_{1}, \varrho_{2}$ and $x \in G$. As easily seen, both $\varrho_{1} \wedge \varrho_{2}, \varrho_{1} \vee \varrho_{2}$ are gauges, and:

$$
\varrho_{1} \wedge \varrho_{2} \leq \varrho_{1}, \varrho_{2} \leq \varrho_{1} \vee \varrho_{2}
$$

Taking for $\mathcal{V}$ the closure of $\mathcal{V}_{0}$ with respect to the operations $\wedge$ and $\vee$, we are done.

Remark The last metrization theorem can be proved even for more general triplets $\left(G, G_{0}, G_{\mathrm{f}}\right)$, consisting of an internal group $G$, a monadic subgroup $G_{0}$ and a galactic subgroup $G_{\mathrm{f}}$, such that $G_{0} \subseteq G_{\mathrm{f}} \subseteq G$, without the assumption that $G_{0}$ is normal in $G_{\mathrm{f}}$. Conversely, any single gauge $\varrho$, as well as any downward directed set $\mathcal{V}$ of admissible size of gauges on an internal group $G$ gives rise to a monadic subgroup $G_{0}$ and a galactic subgroup $G_{\mathrm{f}}$, defined by the formulas from Proposition 1.3.1, such that $G_{0} \subseteq G_{\mathrm{f}} \subseteq G$. However, one cannot prove that $G_{0} \triangleleft G_{\mathrm{f}}$ in general.

Topological groups $\mathbf{G}$ embeddable into observable traces $\mathbf{G} \cong G^{b}$ of IMG group triplets can be easily characterized.

Let $\mathbf{G}$ be a topological group and $\mathbf{U}$ be a symmetric neighborhood of the unit element $1 \in \mathbf{G}$. Then $\mathbf{G}$ is called $\mathbf{U}$-locally uniform if the group multiplication in $\mathbf{G}$ restricted to the set $\mathbf{U} \times \mathbf{U}$ is uniformly continuous in the left (or, equivalently, in the right) uniformity on $\mathbf{G}$ (cf Goldbring [16]). G is locally uniform if it is $\mathbf{U}$-locally uniform for some $\mathbf{U}$.

Obviously, any subgroup of a locally uniform topological group is itself locally uniform (in the subgroup topology).

The easy proof of the following nonstandard formulation of $\mathbf{U}$-local uniformity is left to the reader.

Proposition 1.3.2 Let $\mathbf{G}$ be a topological group and $\mathbf{U}$ be a symmetric neighborhood of the unit element $1 \in \mathbf{G}$. Denote by $\mathbf{N}$ the normalizer of the monad Mon(1) in ${ }^{*} \mathbf{G}$. Then $\mathbf{G}$ is $\mathbf{U}$-locally uniform if and only if ${ }^{*} \mathbf{U} \subseteq \mathbf{N}$. 
Proposition 1.3.3 Let $\mathbf{G}$ be a Hausdorff topological group. Then $\mathbf{G}$ can be embedded into the observable trace $G^{b}=G_{\mathrm{f}} / G_{0}$ of some bounded monadic group $\left(G, G_{0}, G_{\mathrm{f}}\right)$ if and only if $\mathbf{G}$ is locally uniform.

Proof Assume that $\mathbf{G}$ is isomorphic to the observable trace $G^{b}$ of some IMG group triplet $\left(G, G_{0}, G_{\mathrm{f}}\right)$. Then, as $G_{0} \triangleleft G_{\mathrm{f}}$, we have

$$
\left(\forall x_{1}, x_{2}, y_{2}, y_{2} \in G_{\mathrm{f}}\right)\left(x_{1} \approx y_{1} \& x_{2} \approx y_{2} \Rightarrow x_{1} x_{2} \approx y_{1} y_{2}\right)
$$

hence the multiplication in $G$ is $S$-continuous on $G_{\mathrm{f}} \times G_{\mathrm{f}}$, yielding uniform continuity of the multiplication in $\mathbf{G}$ on every set of the form $U^{b} \times U^{b}$, where $U$ is internal and $G_{0} \subseteq U \subseteq G_{\mathrm{f}}$. Since such sets $U^{\mathrm{b}}$ form a neighborhood base of $1 \in \mathbf{G}, \mathbf{G}$ is locally uniform. If $\mathbf{G}$ is just embedded into the observable trace $G^{b}$ then $\mathbf{G}$ is isomorphic to a subgroup of a locally uniform group, hence it is locally uniform, as well.

Now, assume that $\mathbf{G}$ is locally uniform. Let $\kappa$ be the least uncountable cardinal, such that the topology of $\mathbf{G}$ has a base $\mathcal{B}$ of cardinality $<\kappa$, and ${ }^{*} \mathbf{G}$ be a nonstandard extension of $\mathbf{G}$ in a $\kappa$-saturated nonstandard universe. Let $\mathbf{U} \in \mathcal{B}$ be a symmetric neighborhood of $1 \in \mathbf{G}$ such that the group multiplication is uniformly continuous on $\mathbf{U} \times \mathbf{U}$. Denote by $\mathbb{I}^{*} \mathbf{G}$ the monad of the unit element $1 \in{ }^{*} \mathbf{G}$ and by $\mathbf{N}$ its normalizer in ${ }^{*} \mathbf{G}$. Finally we put

$$
\mathbf{S}=\bigcup\left\{{ }^{*} \mathbf{B}: \mathbf{B} \in \mathcal{B} \&{ }^{*} \mathbf{B} \subseteq \mathbf{N}\right\}
$$

and denote by $\mathbb{F}^{*} \mathbf{G}=\langle\mathbf{S}\rangle$ the subgroup of ${ }^{*} \mathbf{G}$ generated by $\mathbf{S}$. Then, obviously, $\mathbb{F}^{*} \mathbf{G}$ is a union of admissibly many internal sets, and ${ }^{*} \mathbf{U} \subseteq \mathbb{F}^{*} \mathbf{G} \subseteq \mathbf{N}$, which means that $\mathbb{I}^{*} \mathbf{G} \triangleleft \mathbb{F}^{*} \mathbf{G}$. The inclusion $\mathbf{G} \subseteq \mathbb{F}^{*} \mathbf{G}$ follows from the fact that, for each $\boldsymbol{a} \in \mathbf{G}$, the group operation is uniformly continuous on $\mathbf{U} \boldsymbol{a} \times \mathbf{U} \boldsymbol{a}$, hence also on $\mathbf{B} \times \mathbf{B}$ where $\mathbf{B} \in \mathcal{B}$ is a neighborhood of $\boldsymbol{a}$ such that $\mathbf{B} \subseteq \mathbf{U} \boldsymbol{a}$.

Thus $\left({ }^{*} \mathbf{G}, \mathbb{I}^{*} \mathbf{G}, \mathbb{F}{ }^{*} \mathbf{G}\right)$ is an IMG group triplet and the star map $\boldsymbol{a} \mapsto{ }^{*} \boldsymbol{a}$ induces an embedding of $\mathbf{G}$ into its observable trace ${ }^{*} \mathbf{G}^{b}=\mathbb{F}^{*} \mathbf{G} / \mathbb{I}^{*} \mathbf{G}$.

For locally compact groups even more can be proved.

Proposition 1.3.4 Let $\mathbf{G}$ be a Hausdorff locally compact topological group. Then, in a sufficiently saturated nonstandard universe, $\mathbf{G}$ is isomorphic to the observable trace $\mathrm{Ns}\left({ }^{*} \mathbf{G}\right) / \operatorname{Mon}(1)$ of the condensing IMG group triplet $\left({ }^{*} \mathbf{G}, \operatorname{Mon}(1), \mathrm{Ns}\left({ }^{*} \mathbf{G}\right)\right)$.

Sketch of proof It suffices to have the nonstandard universe $\kappa$-saturated where $\kappa$ is the least uncountable cardinal bigger than the cardinality of some neighborhood base of $1 \in \mathbf{G}$ and such that $\mathbf{G}$ can be covered by the interiors of less than $\kappa$ compact sets. 
We are mainly interested in condensing IMG group triplets $\left(G, G_{0}, G_{\mathrm{f}}\right)$ with a hyperfinite ambient group $G$. Condensing IMG triplets can be characterized using Proposition 1.2.1. Below $[A: B]$ denotes any of the indices $\lfloor A: B\rfloor,\lfloor A: B\rfloor_{\mathrm{i}}$ or $\lceil A: B\rceil$. The simple proof of the following facts is left to the reader.

Proposition 1.3.5 (a) An IMG group triplet $\left(G, G_{0}, G_{\mathrm{f}}\right)$ is condensing if and only if for any symmetric internal sets $A, B$ between $G_{0}$ and $G_{\mathrm{f}}$ the index $[A: B]$ is finite. If $G$ is hyperfinite then this is equivalent to

$$
0 \not \approx \frac{|A|}{|B|}<\infty
$$

for any internal sets $A, B$ between $G_{0}$ and $G_{\mathrm{f}}$.

(b) The observable trace $G^{b}$ of a condensing group triplet $\left(G, G_{0}, G_{\mathrm{f}}\right)$ is discrete if and only if the subgroup $G_{0}$ is internal; $G^{b}$ is compact if and only if the subgroup $G_{\mathrm{f}}$ is internal.

Obviously, both the bounded monadic groups $\left(* \mathbb{R}, \mathbb{I}^{*} \mathbb{R}, \mathbb{F}^{*} \mathbb{R}\right),\left({ }^{*} \mathbb{C}, \mathbb{I}^{*} \mathbb{C}, \mathbb{F}^{*} \mathbb{C}\right)$ are condensing.

Given a condensing IMG group triplet $\left(G, G_{0}, G_{\mathrm{f}}\right)$ with a hyperfinite ambient group $G$, a positive number $d \in{ }^{*} \mathbb{R}$, such that $d|A| \in \mathbb{F}^{*} \mathbb{R} \backslash \mathbb{I}^{*} \mathbb{R}$ for some (or, equivalently, for each) internal set $A$ between $G_{0}$ and $G_{\mathrm{f}}$, is called a normalizing multiplier or normalizing coefficient for $\left(G, G_{0}, G_{\mathrm{f}}\right)$. According to 1.3 .5 , if $d$ is a normalizing multiplier for $\left(G, G_{0}, G_{\mathrm{f}}\right)$, and $0<d^{\prime} \in{ }^{*} \mathbb{R}$ then $d^{\prime}$ is a normalizing multiplier if and only if $d / d^{\prime} \in \mathbb{F}^{*} \mathbb{R} \backslash \mathbb{I}^{*} \mathbb{R}$. In particular, for any internal set $A$ between $G_{0}$ and $G_{\mathrm{f}}$, $d=1 /|A|$ is a normalizing coefficient for $\left(G, G_{0}, G_{\mathrm{f}}\right)$.

From the results of Section 1.2 it follows directly:

Proposition 1.3.6 Let $\left(G, G_{0}, G_{\mathrm{f}}\right)$ be a condensing IMG group triplet with a hyperfinite ambient group $G$ and a normalizing multiplier $d$. Let $\lambda_{d}$ denote the Loeb measure induced by the constant function $d(x)=d$ on $G$. Then the measure $\boldsymbol{m}_{d}$ obtained by pushing down the Loeb measure $\lambda_{d}$ is both left and right invariant Haar measure on the observable trace $G^{b}=G_{\mathrm{f}} / G_{0}$.

If $\mathbf{G}$ is a locally compact topological group then any pair $(\mathbf{K}, \mathbf{U})$ consisting of a compact set $\mathbf{K} \subseteq \mathbf{G}$ and a symmetric neighborhood $\mathbf{U} \subseteq \mathbf{K}$ of the unit $1 \in \mathbf{G}$ is called a $\mathbf{G}-$ raster. Notice that in such a case the neighborhood $\mathbf{U}$ is relatively compact, while 
$\mathbf{K}$ itself is a compact neighborhood of 1 in $\mathbf{G}$. The system of all $\mathbf{G}-$ rasters, partially ordered by the relation

$$
(\mathbf{K}, \mathbf{U}) \leq(\mathbf{Q}, \mathbf{V}) \Leftrightarrow \mathbf{K} \subseteq \mathbf{Q} \text { and } \mathbf{V} \subseteq \mathbf{U}
$$

is a directed poset. If $(\mathbf{K}, \mathbf{U}) \leq(\mathbf{Q}, \mathbf{V})$, we say that the raster $(\mathbf{Q}, \mathbf{V})$ extends the raster $(\mathbf{K}, \mathbf{U})$. A system of $\mathbf{G}$-rasters $\left(\left(\mathbf{K}_{i}, \mathbf{U}_{i}\right)\right)_{i \in I}$, indexed by some index set $I$, is called a raster base of $\mathbf{G}$ if it is cofinal in the system of all $\mathbf{G}$-rasters, ie, for any $\mathbf{G}$-raster $(\mathbf{K}, \mathbf{U})$, there is a raster $\left(\mathbf{K}_{i}, \mathbf{U}_{i}\right)$ in this system extending $(\mathbf{K}, \mathbf{U})$.

If $\mathbf{G}$ is discrete then it is enough to deal with its raster bases consisting just of pairs $\left(\mathbf{K}_{i},\{1\}\right)$, where $\mathbf{K}_{i}$ are finite sets whose union is $\mathbf{G}$. Similarly, if $\mathbf{G}$ is compact then it is enough to consider its raster bases formed by pairs $\left(\mathbf{G}, \mathbf{U}_{i}\right)$ where the sets $\mathbf{U}_{i}$ form a neighborhood base of $1 \in \mathbf{G}$.

Given a $\mathbf{G}$-raster $(\mathbf{K}, \mathbf{U})$, a mapping $\eta: G \rightarrow \mathbf{G}$ is called a finite $(\mathbf{K}, \mathbf{U})$ approximation or just a $(\mathbf{K}, \mathbf{U})$ approximation of $\mathbf{G}$ if $G$ is a finite group, and $\eta$ satisfies the following two conditions:

$$
\begin{gathered}
(\forall \boldsymbol{x} \in \mathbf{K})(\exists x \in G)(\eta(x) \in \mathbf{U} \boldsymbol{x}) \\
(\forall x, y \in G)(\eta(x), \eta(y) \in \mathbf{K} \Rightarrow \eta(x) \eta(y) \in \mathbf{U} \eta(x y))
\end{gathered}
$$

A $(\mathbf{K}, \mathbf{U})$ approximation is called injective if $\eta$ is an injective mapping; it is called strict if $\eta(1)=1, \eta\left(x^{-1}\right)=\eta(x)^{-1}$ for all $x \in G$, and the second of the above conditions can be strengthened to:

$$
(\forall x, y \in G)(\eta(x), \eta(y) \in \mathbf{K} \Rightarrow \eta(x y)=\eta(x) \eta(y))
$$

Notice that any $(\mathbf{K}, \mathbf{U})$ approximation $\eta: G \rightarrow \mathbf{G}$ satisfies $\eta(1) \in \mathbf{U}$ if $1 \in \eta^{-1}[\mathbf{K}]$, and $\eta\left(x^{-1}\right) \in \mathbf{U} \eta(x)^{-1}$ if $1, x, x^{-1} \in \eta^{-1}[\mathbf{K}]$. Similarly, the strengthened second condition implies $\eta(1)=1$ if $1 \in \eta^{-1}[\mathbf{K}]$, and $\eta\left(x^{-1}\right)=\eta(x)^{-1}$ if $1, x, x^{-1} \in \eta^{-1}[\mathbf{K}]$. However, the convenient conditions $\eta(1)=1, \eta\left(x^{-1}\right)=\eta(x)^{-1}$ alone can always be assumed without loss of generality.

Obviously, if $(\mathbf{K}, \mathbf{U})$ is a $\mathbf{G}$-raster and $\eta: G \rightarrow \mathbf{G}$ is a finite $(\mathbf{K}, \mathbf{U})$ approximation of $\mathbf{G}$ then $\eta$ is a $(\mathbf{Q}, \mathbf{V})$ approximation of $\mathbf{G}$ for any $\mathbf{G}-\operatorname{raster}(\mathbf{Q}, \mathbf{V}) \leq(\mathbf{K}, \mathbf{U})$.

If $(I, \leq)$ is a directed poset then a system of mappings $\left(\eta_{i}: G_{i} \rightarrow \mathbf{G}\right)_{i \in I}$, where each $G_{i}$ is a finite group, is called an approximating system of $\mathbf{G}$ if for every $\mathbf{G}-\operatorname{raster}(\mathbf{K}, \mathbf{U})$ there is $i \in I$ such that $\eta_{j}: G_{j} \rightarrow \mathbf{G}$ is a $(\mathbf{K}, \mathbf{U})$ approximation of $\mathbf{G}$ for each $j \in I$, $j \geq i$.

An internal mapping $\eta: G \rightarrow{ }^{*} \mathbf{G}$ is a hyperfinite infinitesimal approximation, briefly an HFI approximation, of $\mathbf{G}$, if $G$ is a hyperfinite group and:

$$
(\forall \boldsymbol{x} \in \mathbf{G})(\exists x \in G)(\eta(x) \approx \boldsymbol{x})
$$




$$
(\forall x, y \in G)\left(\eta(x), \eta(y) \in \mathrm{Ns}\left({ }^{*} \mathbf{G}\right) \Rightarrow \eta(x y) \approx \eta(x) \eta(y)\right)
$$

The notions of injective and strict HFI approximation, respectively, are defined in the obvious way.

Every hyperfinite infinitesimal approximation $\eta: G \rightarrow{ }^{*} \mathbf{G}$ of a Hausdorff locally compact group $\mathbf{G}$ in a sufficiently saturated nonstandard universe gives rise to an IMG group triplet $\left(G, G_{0}, G_{\mathrm{f}}\right)$, where

$$
\begin{aligned}
& G_{0}=\eta^{-1}[\operatorname{Mon}(1)]=\{x \in G: \eta(x) \approx 1\} \\
& \left.G_{\mathrm{f}}=\eta^{-1}\left[\operatorname{Ns}\left({ }^{*} \mathbf{G}\right)\right]=\left\{x \in G: \eta(x) \in \mathrm{Ns}^{*} \mathbf{G}\right)\right\}
\end{aligned}
$$

then $\mathbf{G}$ is isomorphic to the observable trace $G^{b}=G_{\mathrm{f}} / G_{0}$.

Proposition 1.3.7 Let $\mathbf{G}$ be a Hausdorff locally compact group. Then the following conditions are equivalent:

(i) $\mathbf{G}$ is isomorphic to the observable trace $G^{b}$ of some hyperfinite condensing IMG group triplet $\left(G, G_{0}, G_{\mathrm{f}}\right)$.

(ii) There is an HFI approximation $\eta: G \rightarrow{ }^{*} \mathbf{G}$ of $\mathbf{G}$.

(iii) For every $\mathbf{G}$-raster $(\mathbf{K}, \mathbf{U})$ there is a finite $(\mathbf{K}, \mathbf{U})$ approximation $\eta: G \rightarrow \mathbf{G}$.

(iv) There is an approximating system $\left(\eta_{i}: G_{i} \rightarrow \mathbf{G}\right)_{i \in I}$ of $\mathbf{G}$ by finite groups $G_{i}$ over some directed poset $(I, \leq)$.

Proof (ii) $\Rightarrow$ (i) was already proved, right before formulating the proposition.

(i) $\Rightarrow$ (ii) Assume that $\left(G, G_{0}, G_{\mathrm{f}}\right)$ is a hyperfinite condensing IMG group triplet and $\boldsymbol{\eta}: G_{\mathrm{f}} / G_{0} \rightarrow \mathbf{G}$ is an isomorphism of topological groups. By Proposition 1.2.3, $\boldsymbol{\eta}$ has a lifting $\eta: G \rightarrow{ }^{*} \mathbf{G}$. It is a routine to check that $\eta$ is an HFI approximation of $\mathbf{G}$.

(ii) $\Rightarrow$ (iii) Let $\eta: G \rightarrow{ }^{*} \mathbf{G}$ be an HFI approximation of $\mathbf{G}$. For any $\mathbf{G}-\operatorname{raster}(\mathbf{K}, \mathbf{U})$ denote by $\Phi(\mathbf{K}, \mathbf{U})$ the set of all finite $(\mathbf{K}, \mathbf{U})$ approximations $\phi: F \rightarrow \mathbf{G}$ of $\mathbf{G}$. Then $\eta \in{ }^{*} \Phi(\mathbf{K}, \mathbf{U})$. By the Transfer Principle, $\Phi(\mathbf{K}, \mathbf{U}) \neq \emptyset$.

(iii) $\Rightarrow$ (iv) Denote by $(I, \leq)$ the directed poset set of all $\mathbf{G}$-rasters ordered by the extension relation. Let $\eta_{i}: G_{i} \rightarrow \mathbf{G}$ be a finite $(\mathbf{K}, \mathbf{U})$ approximation of $\mathbf{G}$ for each $i=(\mathbf{K}, \mathbf{U}) \in I$. Clearly, the mappings $\eta_{i}$ form an approximating system of $\mathbf{G}$.

(iv) $\Rightarrow$ (ii) Let $\left(\eta_{i}: G_{i} \rightarrow \mathbf{G}\right)_{i \in I}$ be an approximating system of $\mathbf{G}$ over $(I, \leq)$. Let us embed the situation into a $\kappa$-saturated nonstandard universe, where $\kappa>\aleph_{0}$ and $(I, \leq)$ has a cofinal subset of cardinality $<\kappa$. Then there is $k \in{ }^{*} I$ such that $i \leq k$ for all $i \in I$; it follows that $\eta_{k}: G_{k} \rightarrow{ }^{*} \mathbf{G}$ is an HFI approximation of $\mathbf{G}$. 
Remarks (a) From the above proof it is clear that one can strengthen conditions (ii)-(iv) of Proposition 1.3.7 by requiring injectivity of the corresponding approximation(s), and the modified conditions will be equivalent, again. What is not clear is the way one should modify condition (i) to make it equivalent with them. (Maybe even no modification is needed at all.)

(b) Alternatively, the implication (iv) $\Rightarrow$ (ii) can be proved using the ultraproduct construction with respect to any ultrafilter $\mathcal{D}$ on the directed poset $(I, \leq)$ containing all the sets of the form $\{j \in I: j \geq i\}$ for $i \in I$, applied to the system of finite approximations $\left(\eta_{i}: G_{i} \rightarrow \mathbf{G}\right)_{i \in I}$. More details can be found in Zlatoš [49, Proposition 1.5.8].

The following remark is even of more relevance.

Remark A notion of finite approximability equivalent, for compact metrizable groups, to the above condition (iii) (strengthened in the sense of Remark (a) above) was introduced and studied by Alan Turing [46] already in the late 1930s. In particular, he proved that a compact connected Lie group is approximable by finite groups if and only if it is abelian. Nevertheless, a reasonable characterization of locally compact topological groups isomorphic to observable traces of condensing IMG group triplets with a hyperfinite ambient group is still missing. According to Proposition 1.3.6, every such a group is necessarily unimodular. On the other hand, as a consequence of this theorem of Turing and Proposition 1.3.7, no noncommutative compact (hence unimodular) connected Lie group, like, eg, $\mathrm{SO}(3)$, admits such a representation (see also Gordon and Glebsky [12] and Gordon, Glebsky and Rubio [13]). Similarly, not even all finitely generated discrete groups are approximable by finite groups in this sense (cf Alekseev, Glebsky and Gordon [2], Gordon and Vershik [19]). Fortunately, as we shall see later on, all locally compact abelian groups still behave well.

The following examples (a), (b), (c), as well as some special cases of (d) are essentially taken from Gordon [18]; (b) in fact (without using the present terminology), can be found already in Luxemburg [32].

Example 1.3.8 Let $1 \leq n \in * \mathbb{N}$, and $\mathbb{Z}_{n}=\left\{-\left\lfloor\frac{n-1}{2}\right\rfloor, \ldots,-1,0,1, \ldots,\left\lceil\frac{n-1}{2}\right\rceil\right\}$ be the (hyper)finite cyclic group of order $n$, represented as the set of absolutely smallest remainders modulo $n$.

(a) If $n \in \mathbb{N}$ and $0 \leq k<n / 4$ then the identity mapping $\mathbb{Z}_{n} \rightarrow \mathbb{Z}$ is a strict injective $(K,\{0\})$ approximation of the group $\mathbb{Z}$, where $K=\{0, \pm 1, \ldots, \pm k\}$.

If $n \in{ }^{*} \mathbb{N}_{\infty}$ then the identity mapping $\mathbb{Z}_{n} \rightarrow{ }^{*} \mathbb{Z}$ is a strict HFI approximation of $\mathbb{Z}$. The IMG group triplet arising form it has the form $\left(\mathbb{Z}_{n},\{0\}, \mathbb{Z}\right)$. 
(b) If $n \in \mathbb{N}$ and $r>\pi / n$ then the homomorphism $a \mapsto \mathrm{e}^{2 \pi \mathrm{i} a / n}: \mathbb{Z}_{n} \rightarrow \mathbb{T}$ is a strict injective $(\mathbb{T}, \mathbf{U})$ approximation of the group $\mathbb{T}$, where $\mathbf{U}=\{u \in \mathbb{T}:|\arg u| \leq r\}$.

If $n \in{ }^{*} \mathbb{N}_{\infty}$ then the internal homomorphism $a \mapsto \mathrm{e}^{2 \pi \mathrm{i} a / n}: \mathbb{Z}_{n} \rightarrow{ }^{*} \mathbb{T}$ is a strict injective HFI approximation of $\mathbb{T}$. The corresponding IMG group triplet is $\left(\mathbb{Z}_{n}, G_{0}, \mathbb{Z}_{n}\right)$, where:

$$
G_{0}=\left\{a \in \mathbb{Z}_{n}: a / n \approx 0\right\}
$$

(c) If $n \in \mathbb{N}, 0 \leq k<n / 4 \in \mathbb{N}$, and $d$ and $r>d / 2$ are positive real numbers then the mapping $a \mapsto a d: \mathbb{Z}_{n} \rightarrow \mathbb{R}$ is a strict injective $(\mathbf{K}, \mathbf{U})$ approximation of the group $\mathbb{R}$, where $\mathbf{K}=[-k d, k d], \mathbf{U}=[-r, r]$.

If $n \in *^{*}$, and $d$ is a positive infinitesimal such that $n d \sim \infty$ then the internal mapping $a \mapsto a d: \mathbb{Z}_{n} \rightarrow{ }^{*} \mathbb{R}$ is a strict injective HFI approximation of $\mathbb{R}$, inducing the IMG group triplet $\left(\mathbb{Z}_{n}, G_{0}, G_{\mathrm{f}}\right)$, where:

$$
G_{0}=\left\{a \in \mathbb{Z}_{n}: a d \approx 0\right\} \quad G_{\mathrm{f}}=\left\{a \in \mathbb{Z}_{n}:|a d|<\infty\right\}
$$

(d) Let $\mathbf{G}$ be any Hausdorff locally compact group (written multiplicatively) and $\mathbf{U} \subseteq \mathbf{K} \subseteq \mathbf{G}$ be its compact open subgroups such that $\mathbf{U}$ is normal in $\mathbf{K}$. Then the quotient $G=\mathbf{K} / \mathbf{U}$ is a finite group and any (necessarily injective) mapping $\eta: G \rightarrow \mathbf{G}$ such that $\mathbf{U} \eta(x)=x$ for each $x \in G$ is an injective $(\mathbf{K}, \mathbf{U})$ approximation of $\mathbf{G}$.

If $\mathbf{G}$ admits a raster base $\left(\left(\mathbf{K}_{i}, \mathbf{U}_{i}\right)\right)_{i \in I}$ consisting of compact open subgroups $\mathbf{U}_{i} \triangleleft \mathbf{K}_{i}$ of $\mathbf{G}$ then there are ${ }^{*}$ compact ${ }^{*}$ open subgroups $\mathbf{U} \triangleleft \mathbf{K}$ of ${ }^{*} \mathbf{G}$, such that $\mathbf{U} \subseteq \operatorname{Mon}(1)$, $\mathrm{Ns}\left({ }^{*} \mathbf{G}\right) \subseteq \mathbf{K}$ and the quotient $G=\mathbf{K} / \mathbf{U}$ is a hyperfinite group. Any internal mapping $\eta: G \rightarrow{ }^{*} \mathbf{G}$ such that $\mathbf{U} \eta(x)=x$ for each $x \in G$ is an injective HFI approximation of G. The IMG group triplet $\left(G, G_{0}, G_{\mathrm{f}}\right)$ obtained from $\eta$ satisfies:

$$
\begin{aligned}
& G_{0}=\bigcap_{i \in I} \eta^{-1}\left[{ }^{*} \mathbf{U}_{i}\right]=\left\{\mathbf{U} \boldsymbol{x}:(\forall i \in I)\left(\boldsymbol{x} \in \mathbf{U}_{i}\right)\right\} \\
& G_{\mathrm{f}}=\bigcup_{i \in I} \eta^{-1}\left[{ }^{*} \mathbf{K}_{i}\right]=\left\{\mathbf{U} \boldsymbol{x}:(\exists i \in I)\left(\boldsymbol{x} \in \mathbf{K}_{i}\right)\right\}
\end{aligned}
$$

Both in the finite and in the hyperfinite case, $\eta$ is not a strict approximation, unless it is a genuine homomorphism.

Clearly, if $\eta_{1}: G_{1} \rightarrow \mathbf{G}_{1}$ is a finite $\left(\mathbf{K}_{1}, \mathbf{U}_{1}\right)$ approximation of $\mathbf{G}_{1}$ and $\eta_{2}: G_{2} \rightarrow \mathbf{G}_{2}$ is a finite $\left(\mathbf{K}_{2}, \mathbf{U}_{2}\right)$ approximation of $\mathbf{G}_{2}$ then the mapping $\eta_{1} \times \eta_{2}: G_{1} \times G_{2} \rightarrow \mathbf{G}_{1} \times \mathbf{G}_{2}$ is a finite $\left(\mathbf{K}_{1} \times \mathbf{K}_{2}, \mathbf{U}_{1} \times \mathbf{U}_{2}\right)$ approximation of $\mathbf{G}_{1} \times \mathbf{G}_{2}$. Moreover, it is clear, that $\eta_{1} \times \eta_{2}$ is strict or injective, respectively, once both the approximations $\eta_{1}, \eta_{2}$ have the respective property. Analogous observations apply to HFI approximations as well. 
Thus the items (a), (b) and (c) of the last example enable us to construct strict injective approximating systems and strict injective HFI approximations for all elementary $L C A$ groups, ie for LCA groups of the form $F \times \mathbb{Z}^{k} \times \mathbb{T}^{m} \times \mathbb{R}^{n}$ where $F$ is a finite abelian group and $k, m, n \in \mathbb{N}$.

Example 1.3.8(d) gives a direct hint how to construct both approximating systems and HFI approximations of any LCA group $\mathbf{G}$ with a raster base $\left(\left(\mathbf{K}_{i}, \mathbf{U}_{i}\right)\right)_{i \in I}$ consisting of pairs of subgroups $\mathbf{U}_{i} \subseteq \mathbf{K}_{i}$ of $\mathbf{G}$. The following LCA groups are included as special cases:

1. the compact additive groups of $\tau$-adic integers $\Delta_{\tau}$, where $\tau=\left(\tau_{n}\right)_{n \in \mathbb{N}}$ is any increasing sequence of positive integers such that $\tau_{n} \mid \tau_{n+1}$ for each $n$

2. the additive LCA groups of $\tau$-adic numbers $\mathbb{Q}_{\tau}$, where $\tau=\left(\tau_{n}\right)_{n \in \mathbb{Z}}$ is any sequence of positive integers, such that $\tau_{n}<\tau_{n+1}, \tau_{n} \mid \tau_{n+1}$ for $n \geq 0$, and $\tau_{n}<\tau_{n-1}, \tau_{n} \mid \tau_{n-1}$ for $n \leq 0$

3. torsion (discrete abelian) groups, as they are direct (inductive) limits of finite abelian groups

4. profinite (compact abelian) groups, ie, inverse (projective) limits of finite abelian groups $^{2}$

In Gordon [18] also HFI approximations of $\tau$-adic solenoids

$$
\Sigma_{\tau}=\left(\mathbb{R} \times \Delta_{\tau}\right) /\{(a, a): a \in \mathbb{Z}\}
$$

which do not fall within the scope of Example 1.3.8(d), are described.

Actually, any LCA group admits arbitrarily good finite approximations and, henceforth, HFI approximations, too. The key ingredient of the proof of this fact is the following structure theorem for compactly generated LCA groups (ie, LCA groups generated by some compact subset). Its proof is relatively elementary (though certainly not trivial), in particular, it does not rely on the Pontraygin-van Kampen Duality Theorem (cf Pontryagin [38, Theorem 50], Hewitt and Ross [24, Theorem 9.6]).

Proposition 1.3.9 Let $\mathbf{G}$ be a compactly generated Hausdorff LCA group. Then every compact neighborhood $\mathbf{V}$ of 0 in $\mathbf{G}$ contains a closed subgroup $\mathbf{H}$ of $\mathbf{G}$ such that the quotient $\mathbf{G} / \mathbf{H}$ is an elementary LCA group.

Theorem 1.3.10 (The Finite LCA Group Approximation Theorem) Let $\mathbf{G}$ be a Hausdorff LCA group. Then, for any $\mathbf{G}-\operatorname{raster}(\mathbf{K}, \mathbf{U})$, there is a finite abelian group $G$ and an injective $(\mathbf{K}, \mathbf{U})$ approximation $\eta: G \rightarrow \mathbf{G}$ of $\mathbf{G}$, such that $\eta(0)=0$ and

\footnotetext{
${ }^{2}$ Obviously, 1 . is a special case of 4 .
} 
$\eta(-a)=-\eta(a)$ for each $a \in G$. Equivalently, $\mathbf{G}$ admits some approximating system $\left(\eta_{i}: G_{i} \rightarrow \mathbf{G}\right)_{i \in I}$ by finite abelian groups, such that all the mappings $\eta_{i}$ are injective and preserve 0 and inverses.

Proof Let us pick a compact symmetric neighborhood $\mathbf{V}$ of 0 such that $\mathbf{V}+\mathbf{V} \subseteq \mathbf{U}$. According to the above Proposition 1.3.10, $\mathbf{V}$ contains a closed subgroup $\mathbf{H}$ such that the quotient $\mathbf{E}=\langle\mathbf{K}\rangle / \mathbf{H}$ of the subgroup $\langle\mathbf{K}\rangle$ of $\mathbf{G}$ generated by the compact set $\mathbf{K}$ is an elementary LCA group. Denote by $\mathbf{V}^{\prime}$ and $\mathbf{K}^{\prime}$ the images of the sets $\mathbf{V}, \mathbf{K}$, respectively, under the canonical projection $\psi:\langle\mathbf{K}\rangle \rightarrow \mathbf{E}$. Then $\mathbf{V}^{\prime}$ is a symmetric neighborhood of 0 and $\mathbf{K}^{\prime}$ is a compact set in $\mathbf{E}$. According to Example 1.3.8 and the comment following it, there is a finite group $G$ and a strict injective $\left(\mathbf{K}^{\prime}, \mathbf{V}^{\prime}\right)$ approximation $\zeta: G \rightarrow \mathbf{E}$. Let $\sigma: \mathbf{E} \rightarrow\langle\mathbf{K}\rangle$ be any (necessarily injective) mapping such that $\sigma(0)=0, \sigma(-x)=-\sigma(x)$ and $\psi(\sigma(x))=x$ for $x \in \mathbf{E}$. Then $\eta=\sigma \circ \zeta: G \rightarrow \mathbf{G}$ is an injective mapping, satisfying $\eta(0)=0$, and $\eta(-a)=-\eta(a)$ for $a \in G$. Straightforward arguments show that

$$
\mathbf{K} \subseteq \mathbf{K}+\mathbf{H} \subseteq \eta[G]+\mathbf{V}+\mathbf{H} \subseteq \eta[G]+\mathbf{U}
$$

and

$$
\eta(a)+\eta(b)-\eta(a+b) \in \mathbf{H} \subseteq \mathbf{V} \subseteq \mathbf{U}
$$

for $a, b \in G$, whenever $\eta(a), \eta(b), \eta(a+b) \in \mathbf{K}+\mathbf{H}$. Hence, $\eta$ is a $(\mathbf{K}, \mathbf{U})$ approximation of $\mathbf{G}$. The equivalence of the first and the second formulation is obvious in view of Proposition 1.3.7.

Corollary 1.3.11 (The Hyperfinite LCA Group Approximation Theorem) Let G be a Hausdorff LCA group. Then, in a sufficiently saturated nonstandard universe, there is an internal hyperfinite abelian group $G$ and an injective HFI approximation $\eta: G \rightarrow{ }^{*} \mathbf{G}$ of $\mathbf{G}$, such that $\eta(0)=0$ and $\eta(-a)=-\eta(a)$ for $a \in G$. It follows that $\mathbf{G}$ is isomorphic to the observable trace $G^{b}=G_{\mathrm{f}} / G_{0}$ of the IMG group triplet $\left(G, G_{0}, G_{\mathrm{f}}\right)$ with a hyperfinite abelian ambient group $G$, where $G_{0}=\eta^{-1}[\operatorname{Mon}(0)]$ and $G_{\mathrm{f}}=\eta^{-1}\left[\mathrm{Ns}\left({ }^{*} \mathbf{G}\right)\right]$.

\section{Pontryagin-van Kampen duality in the hyperfinite setting}

In this chapter we finally come to the central topic of our paper, which is the study of condensing IMG group triplets with a hyperfinite abelian ambient group and their relation to the Pontryagin-van Kampen Duality Theorem. In particular, we will prove the first and the second of the three Gordon's conjectures concerning them. However, 
we start with a technical section dealing mainly with the additive combinatorial structure of some sets in finite abelian groups.

\subsection{Fourier transforms, Bohr sets and spectral sets in finite abelian groups}

In this section we establish some inclusions between certain types of subsets of finite abelian groups and their dual groups, as well some estimates of their size. Later on, we will apply them within the realm of hyperfinite abelian groups by means of the Transfer Principle. To this end we will systematically employ the discrete Fourier transform as our main tool. The results thus obtained will not depend on the normalizing coefficients occurring in it. As a matter of convenience we choose $d=1 /|G|$ and $\widehat{d}=1$.

In what follows $G$ denotes a finite abelian group with the addition operation. The operation on its dual group $\widehat{G}$ is written as the multiplication. The set $\mathbb{C}^{G}$ of all functions $G \rightarrow \mathbb{C}$ becomes a unitary space endowed with the Hermitian inner product, given as the expectation

$$
\langle f, g\rangle_{G}=\mathrm{E}(f \cdot \bar{g})=\mathrm{E}_{x \in G} f(x) \bar{g}(x)=\frac{1}{|G|} \sum_{x \in G} f(x) \overline{g(x)}
$$

for $f, g \in \mathbb{C}^{G}$, and the corresponding $\mathrm{L}^{2}$-norm:

$$
\|f\|_{2}=\sqrt{\langle f, f\rangle_{G}}=\sqrt{\mathrm{E}(f \cdot \bar{f})}
$$

The dual group $\widehat{G}=\operatorname{Hom}(G, \mathbb{T})$, considered as a subset of $\mathbb{C}^{G}$, forms an orthonormal basis of $\mathbb{C}^{G}$. Thus for the Fourier transform $\mathcal{F}: \mathbb{C}^{G} \rightarrow \mathbb{C}^{\widehat{G}}$

$$
\mathcal{F}(f)(\gamma)=\widehat{f}(\gamma)=\langle f, \gamma\rangle_{G}=\mathrm{E}(f \cdot \bar{\gamma})
$$

the Fourier inversion formula takes the form:

$$
f=\sum_{\gamma \in \widehat{G}} \widehat{f}(\gamma) \gamma
$$

The Hermitian inner product on $\mathbb{C}^{\widehat{G}}$

$$
\langle\varphi, \psi\rangle_{\widehat{G}}=\sum_{\gamma \in \widehat{G}} \varphi(\gamma) \bar{\psi}(\gamma)
$$

ensures the Plancherel identity:

$$
\langle f, g\rangle_{G}=\langle\widehat{f}, \widehat{g}\rangle_{\widehat{G}}
$$

The convolution on $\mathbb{C}^{G}$ is also defined as the expectation:

$$
(f * g)(x)=\mathrm{E}_{y \in G} f(x-y) g(y)
$$


For its Fourier transform we have:

$$
\widehat{f * g}=\widehat{f} \cdot \widehat{g}
$$

Additionally, we will make use of the $\mathrm{L}^{p}$-norms on $\mathbb{C}^{G}$ and the $\ell^{p}$-norms on $\mathbb{C}^{\widehat{G}}$

$$
\begin{gathered}
\|f\|_{p}=\left(\mathrm{E}|f|^{p}\right)^{1 / p}=\left(\mathrm{E}_{x \in G}|f(x)|^{p}\right)^{1 / p}=\left(\frac{1}{|G|} \sum_{x \in G}|f(x)|^{p}\right)^{1 / p} \\
\|\varphi\|_{p}=\left(\sum_{\chi \in \widehat{G}}|\varphi(\chi)|^{p}\right)^{1 / p}
\end{gathered}
$$

for $1 \leq p<\infty$, as well as of the $\mathrm{L}^{\infty}$-norm on $\mathbb{C}^{G}$ and the $\ell^{\infty}$-norm on $\mathbb{C}^{\widehat{G}}$

$$
\|f\|_{\infty}=\max _{x \in G}|f(x)| \quad\|\varphi\|_{\infty}=\max _{\chi \in \widehat{G}}|\varphi(\chi)|
$$

for $f \in \mathbb{C}^{G}, \varphi \in \mathbb{C}^{\widehat{G}}$.

Let us list some well known and/or obvious relations:

$$
\|\widehat{f}\|_{2}=\|f\|_{2} \quad\|\widehat{f}\|_{\infty} \leq\|f\|_{1}
$$

which are special cases of the Hausdorff-Young inequality

$$
\|\widehat{f}\|_{q} \leq\|f\|_{p}
$$

for $1 \leq p \leq 2$ and $q$ being the dual exponent of $p$, ie, $1 / p+1 / q=1$.

We denote by $f_{a}$ the shift of the function $f: G \rightarrow \mathbb{C}$ by the element $a \in G$, ie

$$
f_{a}(x)=f(x-a)
$$

for $x \in G$. Then

$$
\widehat{f_{a}}(\gamma)=\bar{\gamma}(a) \widehat{f}(\gamma) \quad \text { and } \quad \widehat{f}_{\gamma}=\widehat{\gamma f}
$$

for $\gamma \in \widehat{G}$, as well as

$$
f * g=\mathrm{E}_{a \in G} f_{a} g(a)=\mathrm{E}_{a \in G} f(a) g_{a}
$$

and

$$
(f * g)_{a}=f * g_{a}=f_{a} * g
$$

for $f, g \in \mathbb{C}^{G}$.

A norm $\mathrm{N}$ on the linear space $\mathbb{C}^{G}$ is called translation invariant if $\mathrm{N}\left(f_{a}\right)=\mathrm{N}(f)$ for all $f \in \mathbb{C}^{G}, a \in G$. For any translation invariant norm $\mathrm{N}$ we have:

$$
\mathrm{N}(f * g) \leq \mathrm{N}(f)\|g\|_{1}
$$

Journal of Logic \& Analysis 13:1 (2021) 
A norm $\mathrm{N}$ on $\mathbb{C}^{G}$ is called absolute if $\mathrm{N}(f) \leq \mathrm{N}(g)$ for any functions $f, g \in \mathbb{C}^{G}$ such that $|f(x)| \leq|g(x)|$ for all $x \in G$. For any absolute norm $\mathrm{N}$ we have:

$$
\mathrm{N}(f g) \leq \mathrm{N}(f)\|g\|_{\infty}
$$

As all the norms $\|\cdot\|_{p}$ are obviously translation invariant and absolute,

$$
\|f * g\|_{p} \leq\|f\|_{p}\|g\|_{1} \quad\|f g\|_{p} \leq\|f\|_{p}\|g\|_{\infty}
$$

for $f, g: G \rightarrow \mathbb{C}, 1 \leq p \leq \infty$. If $f, g: G \rightarrow \mathbb{R}$ are both nonnegative on $G$ then even:

$$
\|f * g\|_{1}=\|f\|_{1}\|g\|_{1}
$$

For the characteristic function (indicator) $1_{A}$ of a set $A \subseteq G$ and $1 \leq p<\infty$ we have:

$$
\left\|1_{A}\right\|_{p}^{p}=\left\|1_{A}\right\|_{2}^{2}=\left\|1_{A}\right\|_{1}=\frac{|A|}{|G|}
$$

If $f: G \rightarrow \mathbb{C}$ is even, ie $f(-x)=f(x)$ for $x \in G$, then so is $\widehat{f}: \widehat{G} \rightarrow \mathbb{C}$ and we have:

$$
\widehat{f}(\gamma)=\mathrm{E}_{x \in G} f(x) \operatorname{Re} \gamma(x) \quad f(x)=\sum_{\gamma \in \widehat{G}} \widehat{f}(\gamma) \operatorname{Re} \gamma(x)
$$

The support of a function $g: G \rightarrow \mathbb{C}$ is defined as the set:

$$
\operatorname{supp} g=\{x \in G: g(x) \neq 0\}
$$

The following estimate generalizes an inequality in Green and Ruzsa [20], which was part of the proof there of Proposition 3.1, from indicators to arbitrary nonnegative functions.

Lemma 2.1.1 Let $f: G \rightarrow \mathbb{R}$ be nonnegative, and $D \subseteq G$ be a nonempty set such that $\operatorname{supp}(f * f) \subseteq D$. Then:

$$
\sum_{\gamma \in \widehat{G}}|\widehat{f}(\gamma)|^{4} \geq \frac{\|f\|_{1}^{4}}{\left\|1_{D}\right\|_{1}}
$$

Proof By the Plancherel identity and the relation between the Fourier transform and the convolution,

$$
\sum_{\gamma \in \widehat{G}}|\widehat{f}(\gamma)|^{4}=\left\langle\widehat{f}^{2}, \widehat{f}^{2}\right\rangle_{\widehat{G}}=\langle f * f, f * f\rangle_{G}=\|f * f\|_{2}^{2}
$$

for any function $f: G \rightarrow \mathbb{C}$. Using Cauchy-Schwartz inequality, the fact that $f * f$ is supported on $D$, and the nonnegativity of $f$, we get:

$$
\|f * f\|_{2}^{2}\left\|1_{D}\right\|_{2}^{2} \geq\left|\left\langle f * f, 1_{D}\right\rangle_{G}\right|^{2}=\|f * f\|_{1}^{2}=\|f\|_{1}^{4}
$$

The claim follows immediately. 
For any subsets $A \subseteq G, \Gamma \subseteq \widehat{G}$ and any $\alpha \in * \mathbb{R}, 0 \leq \alpha \leq \pi$, we introduce the Bohr sets (cf Tao and $\mathrm{Vu}[44])$ :

$$
\begin{aligned}
& \operatorname{Bohr}_{\alpha}(A)=\{\gamma \in \widehat{G}:(\forall a \in A)(|\arg \gamma(a)| \leq \alpha)\} \\
& \operatorname{Bohr}_{\alpha}(\Gamma)=\{a \in G:(\forall \gamma \in \Gamma)(|\arg \gamma(a)| \leq \alpha)\}
\end{aligned}
$$

Obviously,

$$
\begin{aligned}
\operatorname{Bohr}_{\alpha}(A) & =\{\gamma \in \widehat{G}:(\forall a \in A)(|\gamma(a)-1| \leq 2 \sin (\alpha / 2))\} \\
& =\{\gamma \in \widehat{G}:(\forall a \in A)(\operatorname{Re} \gamma(a) \geq \cos \alpha)\}
\end{aligned}
$$

and similarly for $\operatorname{Bohr}_{\alpha}(\Gamma)$. We also have

$$
\operatorname{Bohr}_{\beta}(A) \subseteq \operatorname{Bohr}_{\alpha}(A) \quad \text { and } \quad \operatorname{Bohr}_{\beta}(\Gamma) \subseteq \operatorname{Bohr}_{\alpha}(\Gamma)
$$

for $0 \leq \beta \leq \alpha \leq \pi$. For any fixed $\alpha$, the assignments $A \mapsto \operatorname{Bohr}_{\alpha}(A), \Gamma \mapsto \operatorname{Bohr}_{\alpha}(\Gamma)$ form a Galois connection, ie

$$
A \subseteq \operatorname{Bohr}_{\alpha}\left(\operatorname{Bohr}_{\alpha}(A)\right) \quad \text { and } \quad \operatorname{Bohr}_{\alpha}(B) \subseteq \operatorname{Bohr}_{\alpha}(A)
$$

whenever $A \subseteq B \subseteq G$ and similarly for $\Gamma \subseteq \Delta \subseteq \widehat{G}$.

The spectral set or the spectrum of a function $f: G \rightarrow \mathbb{C}$ at the threshold $t \in \mathbb{R}$ is the set:

$$
\operatorname{Spec}_{t}(f)=\left\{\gamma \in \widehat{G}:|\widehat{f}(\gamma)| \geq t\|f\|_{1}\right\} \subseteq \widehat{G}
$$

This is a slight generalization of a definition from Tao and $\mathrm{Vu}[44, \S 4.6]$ were spectral sets of subsets $A \subseteq G$ were defined by:

$$
\operatorname{Spec}_{t}(A)=\operatorname{Spec}_{t}\left(1_{A}\right)=\left\{\gamma \in \widehat{G}:\left|\widehat{1_{A}}(\gamma)\right| \geq t\left\|1_{A}\right\|_{1}\right\}
$$

As $|\widehat{f}(\gamma)| \leq\|\widehat{f}\|_{\infty} \leq\|f\|_{1}, \operatorname{Spec}_{t}(f)=\emptyset$ whenever $t>1$ and $f$ is not identically 0 ; similarly, $\operatorname{Spec}_{t}(f)=\widehat{G}$ for $t \leq 0$. Thus it makes sense to consider the spectral sets just for the threshold values $0 \leq t \leq 1$. Also the following implication is trivial:

$$
s \leq t \Rightarrow \operatorname{Spec}_{t}(f) \subseteq \operatorname{Spec}_{s}(f)
$$

Lemma 2.1.2 Let $f: G \rightarrow \mathbb{R}$ be an even nonnegative function, $D \subseteq G$ be a nonempty set such that $\operatorname{supp} f \subseteq D$, and $0 \leq \alpha \leq \pi / 2$. Then

$$
\operatorname{Bohr}_{\alpha}(D) \subseteq \operatorname{Spec}_{t}(f)
$$

whenever $0 \leq t \leq \cos \alpha$. 
Proof Take any $\gamma \in \operatorname{Bohr}_{\alpha}(D)$. According to the assumptions on $f, t$ and $\alpha$ we have

$$
\begin{aligned}
|\widehat{f}(\gamma)| & =\left|\mathrm{E}_{x \in G} f(x) \bar{\gamma}(x)\right|=\left|\mathrm{E}_{x \in G} 1_{D}(x) f(x) \operatorname{Re} \gamma(x)\right| \\
& \geq \mathrm{E}_{x \in G} f(x) \cos \alpha=\|f\|_{1} \cos \alpha \geq t\|f\|_{1}
\end{aligned}
$$

thus $\gamma \in \operatorname{Spec}_{t}(f)$.

The following result generalizes an inclusion proved in Green and Ruzsa [20] during the proof of Proposition 3.1, as well.

Proposition 2.1.3 Let $f: G \rightarrow \mathbb{R}$ be a nonnegative function, not identically equal to 0 , $D \subseteq G$ be a nonempty set such that $\operatorname{supp}(f * f) \subseteq D$, and $0<\alpha<\pi / 2,0 \leq t \leq 1$. Then

$$
\operatorname{Bohr}_{\alpha}\left(\operatorname{Spec}_{t}(f)\right) \subseteq D-D
$$

whenever:

$$
t \leq \frac{\|f\|_{1}}{\|f\|_{2}\left\|1_{D}\right\|_{2}} \cdot \sqrt{\frac{\cos \alpha}{1+\cos \alpha}}
$$

Proof Let $f_{-}$denote the function given by $f_{-}(x)=f(-x)$; since $f: G \rightarrow \mathbb{R}$, we have $\widehat{f_{-}}(\gamma)=\widehat{\widehat{f}(\gamma)}$ and $|\widehat{f}(\gamma)|^{2}=\widehat{f * f_{-}}(\gamma)$. Then both the functions $f * f_{-}, f * f * f_{-} * f_{-}$ are even, and:

$$
\operatorname{supp}\left(f * f * f_{-} * f_{-}\right) \subseteq D-D
$$

Let $x \in \operatorname{Bohr}_{\alpha}\left(\operatorname{Spec}_{t}(f)\right)$. It suffices to prove that $\left(f * f * f_{-} * f_{-}\right)(x)>0$. Putting $\Gamma=\operatorname{Spec}_{t}(f)$, we have $\operatorname{Re} \gamma(x) \geq \cos \alpha$ for $\gamma \in \Gamma$. By the Fourier inversion formula, Lemma 2.1.1 and Plancherel identity we get:

$$
\begin{aligned}
\left(f * f * f_{-} * f_{-}\right)(x) & =\sum_{\gamma \in \widehat{G}}|\widehat{f}(\gamma)|^{4} \gamma(x) \\
& =\sum_{\gamma \in \Gamma}|\widehat{f}(\gamma)|^{4} \operatorname{Re} \gamma(x)+\sum_{\gamma \in \widehat{G} \backslash \Gamma}|\widehat{f}(\gamma)|^{4} \operatorname{Re} \gamma(x) \\
& >\sum_{\gamma \in \Gamma}|\widehat{f}(\gamma)|^{4} \cos \alpha-\sum_{\gamma \in \widehat{G} \backslash \Gamma}|\widehat{f}(\gamma)|^{4} \\
& =\sum_{\gamma \in \widehat{G}}|\widehat{f}(\gamma)|^{4} \cos \alpha-\sum_{\gamma \in \widehat{G} \backslash \Gamma}|\widehat{f}(\gamma)|^{4}(1+\cos \alpha) \\
& \geq \frac{\|f\|_{1}^{4}}{\left\|1_{D}\right\|_{1}} \cos \alpha-\max _{\gamma \in \widehat{G} \backslash \Gamma}|\widehat{f}(\gamma)|^{2} \sum_{\gamma \in \widehat{G}}|\widehat{f}(\gamma)|^{2}(1+\cos \alpha)
\end{aligned}
$$




$$
\begin{aligned}
& \geq \frac{\|f\|_{1}^{4}}{\left\|1_{D}\right\|_{1}} \cos \alpha-t^{2}\|f\|_{1}^{2}\|f\|_{2}^{2}(1+\cos \alpha) \\
& =\|f\|_{1}^{2}\left(\frac{\|f\|_{1}^{2}}{\left\|1_{D}\right\|_{2}^{2}} \cos \alpha-t^{2}\|f\|_{2}^{2}(1+\cos \alpha)\right)
\end{aligned}
$$

The strict inequality in the third line is due to the fact that for the trivial character $1_{G} \in \Gamma$ we have $\operatorname{Re} 1_{G}(x)=1>\cos \alpha$, since $\alpha>0$. According to the assumption on $t$, the expression in the last line is $\geq 0$.

Remarks (a) It would be enough to have the above result for a single fixed value $\alpha$. The authors both in Green and Ruzsa [20] as well as in Tao and Vu [44] take $\alpha=\pi / 3$, which, in our case, would result in the estimate:

$$
t \leq \frac{\|f\|_{1}}{\|f\|_{2}\left\|1_{D}\right\|_{2} \sqrt{3}}
$$

(b) It is worthwhile to notice that in the special case of $f$ being the indicator of a nonempty set $A \subseteq G$ and $D=A+A$ we have

$$
\frac{\|f\|_{1}}{\|f\|_{2}\left\|1_{D}\right\|_{2}}=\frac{\left\|1_{A}\right\|_{1}}{\left\|1_{A}\right\|_{2}\left\|1_{A+A}\right\|_{2}}=\sqrt{\frac{|A|}{|A+A|}}=\frac{1}{\sqrt{\sigma(A)}}
$$

where $\sigma(A)=|A+A| /|A|$ is the doubling constant of $A$.

We will need also some lower and upper bounds of the size of spectral sets of some functions.

Proposition 2.1.4 Let $f: G \rightarrow \mathbb{R}$ be a nonnegative function, not identically equal to $0, D \subseteq G$ be a nonempty set such that $\operatorname{supp}(f * f) \subseteq D$, and $0<t \leq 1$. Then:

$$
\frac{|G|}{|D|}-t^{2} \frac{\|f\|_{2}^{2}}{\|f\|_{1}^{2}} \leq\left|\operatorname{Spec}_{t}(f)\right| \leq \frac{1}{t^{2}} \frac{\|f\|_{2}^{2}}{\|f\|_{1}^{2}}
$$

Proof Let us denote $\Gamma=\operatorname{Spec}_{t}(f)$. According to Lemma 2.1.1 we have

$$
\begin{aligned}
\frac{\|f\|_{1}^{4}}{\left\|1_{D}\right\|_{1}} \leq \sum_{\gamma \in \widehat{G}}|\widehat{f}(\gamma)|^{4} & =\sum_{\gamma \in \Gamma}|\widehat{f}(\gamma)|^{4}+\sum_{\gamma \in \widehat{G} \backslash \Gamma}|\widehat{f}(\gamma)|^{4} \\
& \leq|\Gamma|\|\widehat{f}\|_{\infty}^{4}+t^{2}\|f\|_{1}^{2} \sum_{\gamma \in \widehat{G} \backslash \Gamma}|\widehat{f}(\gamma)|^{2} \\
& \leq|\Gamma|\|f\|_{1}^{4}+t^{2}\|f\|_{1}^{2}\|f\|_{2}^{2}
\end{aligned}
$$

using Plancherel formula to pass to the last line. This gives the lower bound. 
The upper bound readily follows from the following computation:

$$
\|f\|_{2}^{2}=\|\widehat{f}\|_{2}^{2}=\sum_{\gamma \in \widehat{G}}|\widehat{f}(\gamma)|^{2} \geq \sum_{\gamma \in \Gamma}|\widehat{f}(\gamma)|^{2} \geq t^{2}\|f\|_{1}^{2}|\Gamma|
$$

Remark Notice that the lower bound is relevant just in case

$$
t<\frac{\|f\|_{1}}{\|f\|_{2}\left\|1_{D}\right\|_{2}}
$$

otherwise it is trivial. One of its consequences can be stated as

$$
\frac{|\operatorname{supp}(f * f)|\left|\operatorname{Spec}_{t}(f)\right|}{|G|} \geq 1-t^{2} \frac{|\operatorname{supp}(f * f)|}{|G|} \frac{\|f\|_{2}^{2}}{\|f\|_{1}^{2}} \geq 1-t^{2} \frac{\|f\|_{2}^{2}}{\|f\|_{1}^{2}}
$$

in which form it can be regarded as a kind of the Uncertainty Principle, setting some lower bound for the expression on the left. For $f=1_{A}$ being the indicator of a nonempty set $A \subseteq G$ the first inequality gives

$$
\frac{|A+A|\left|\operatorname{Spec}_{t}(A)\right|}{|G|} \geq 1-t^{2} \sigma(A)
$$

Let us close this technical section with a kind of the Smoothness-and-Decay Principle indicating that spectral sets of functions continuous in some sense tend to avoid discontinuous characters. We will devote a whole section to this topic in our next paper [50].

Proposition 2.1.5 Let $f: G \rightarrow \mathbb{C}$ be not identically equal to 0 and $C, D \subseteq G$ satisfy $\operatorname{supp} f \cup(\operatorname{supp} f+C) \subseteq D \neq \emptyset$. Let $0<t \leq 1,0<\alpha<\pi$ and $\varepsilon>0$ be real numbers. Assume that

$$
\varepsilon \leq 2 t \frac{\|f\|_{1}}{\left\|1_{D}\right\|_{1}} \sin \frac{\alpha}{2}
$$

and $\left\|f_{a}-f\right\|_{\infty} \leq \varepsilon$ for $a \in C$. Then $\operatorname{Spec}_{t}(f) \subseteq \operatorname{Bohr}_{\alpha}(C)$.

Proof If $a \in C$ then $\operatorname{supp}\left(f_{a}-f\right) \subseteq(\operatorname{supp} f+C) \cup \operatorname{supp} f \subseteq D$, hence:

$$
\left\|\widehat{f_{a}}-\widehat{f}\right\|_{\infty} \leq\left\|f_{a}-f\right\|_{1}=\mathrm{E}_{x \in G}\left|f_{a}(x)-f(x)\right| \leq \varepsilon\left\|1_{D}\right\|_{1}
$$

Now, take any $\gamma \in \operatorname{Spec}_{t}(f)$ and assume that $\gamma \notin \operatorname{Bohr}_{\alpha}(C)$. Then there is $a \in C$ such that $|\gamma(a)-1|>2 \sin (\alpha / 2)$. Thus we have

$$
\varepsilon\left\|1_{D}\right\|_{1} \geq\left\|\widehat{f_{a}}-\widehat{f}\right\|_{\infty} \geq\left|\widehat{f_{a}}(\gamma)-\widehat{f}(\gamma)\right|=|\bar{\gamma}(a)-1||\widehat{f}(\gamma)|>2 t\|f\|_{1} \sin \frac{\alpha}{2}
$$

contradicting the assumed upper bound for $\varepsilon$. 
A brief inspection of the proof yields the following modification of the last result.

Corollary 2.1.6 Let $f: G \rightarrow \mathbb{C}$ be not identically equal to $0, C \subseteq G$, and $0<t \leq 1$, $0<\alpha<\pi, \varepsilon>0$ be real numbers. Assume that $\varepsilon \leq 2 t \sin \frac{\alpha}{2}$, and $\left\|f_{a}-f\right\|_{1} \leq \varepsilon\|f\|_{1}$ for $a \in C$. Then $\operatorname{Spec}_{t}(f) \subseteq \operatorname{Bohr}_{\alpha}(C)$.

\subsection{The dual triplet}

Throughout this and the next section, $\left(G, G_{0}, G_{\mathrm{f}}\right)$ denotes a fixed but arbitrary condensing IMG group triplet with a hyperfinite abelian ambient group $G$ (tacitly assumed to be externally infinite). ${ }^{3}$ We also fix a system $\mathcal{Q}$ of admissible size, directed both downward and upward, consisting of symmetric internal sets such that $G_{0}=\bigcap \mathcal{Q}$ and $G_{\mathrm{f}}=\bigcup \mathcal{Q}$.

Let us denote by $\mathbf{G}=G^{b}=G_{\mathrm{f}} / G_{0}$ the observable trace of the triplet. The LCA group $\mathbf{G}$ gives rise to the dual group $\widehat{\mathbf{G}}$ of all continuous homomorphisms (characters) $\gamma: \mathbf{G} \rightarrow \mathbb{T}$, endowed with the compact-open topology. Then $\widehat{\mathbf{G}}$, as an LCA group, can itself be represented as the observable trace of some condensing IMG group triplet $\left(H, H_{0}, H_{\mathrm{f}}\right)$ with a hyperfinite abelian ambient group $H$. One can naturally expect that at least one from among the triplets representing the dual group $\widehat{\mathbf{G}}$ can be constructed from the original triplet $\left(G, G_{0}, G_{\mathrm{f}}\right)$ in some canonical way.

Let $\widehat{G}=\operatorname{Hom}\left(G,{ }^{*} \mathbb{T}\right)$ denote the set of all internal homomorphisms (characters) $\gamma: G \rightarrow{ }^{*} \mathbb{T}$; then $\widehat{G}$ with the pointwise multiplication is a hyperfinite abelian group internally isomorphic to $G$ (though not in a canonical way). For any sets $A \subseteq G$, $\Gamma \subseteq \widehat{G}$ we define their infinitesimal annihilators by:

$$
\begin{aligned}
& A^{\downarrow}=\{\gamma \in \widehat{G}:(\forall a \in A)(\gamma(a) \approx 1)\} \\
& \Gamma^{\downarrow}=\{a \in G:(\forall \gamma \in \Gamma)(\gamma(a) \approx 1)\}
\end{aligned}
$$

Obviously, $A^{\sim}$ is a subgroup of $\widehat{G}$ and $\Gamma^{\sim}$ is a subgroup of $G$. For any $A, B \subseteq G$, $\Gamma, \Delta \subseteq \widehat{G}$ we have

$$
A \subseteq \Gamma^{\downarrow} \Leftrightarrow \Gamma \subseteq A^{\downarrow}
$$

as well as

$$
\begin{aligned}
& A \subseteq B \Rightarrow B^{\downarrow} \subseteq A^{\nu} \quad \text { and } \quad A \subseteq A^{\nu \downarrow} \\
& \Gamma \subseteq \Delta \Rightarrow \Delta^{\nu} \subseteq \Gamma^{\downarrow} \quad \text { and } \quad \Gamma \subseteq \Gamma^{\downarrow \nu}
\end{aligned}
$$

\footnotetext{
${ }^{3}$ However, the reader should keep in mind that some of our accounts remain valid for general internal abelian ambient group $G$, as well, or require just some minor modification.
} 
showing that the assignments $A \mapsto A^{\downarrow}, \Gamma \mapsto \Gamma^{\downarrow}$ form a Galois connection.

We are particularly interested in the subgroups

$$
\begin{aligned}
& G_{0}^{\downarrow}=\left\{\gamma \in \widehat{G}:\left(\forall x \in G_{0}\right)(\gamma(x) \approx 1)\right\} \\
& G_{\mathrm{f}}^{\downarrow}=\left\{\gamma \in \widehat{G}:\left(\forall x \in G_{\mathrm{f}}\right)(\gamma(x) \approx 1)\right\}
\end{aligned}
$$

of the dual group $\widehat{G}$. As every $\gamma \in \widehat{G}$ is a group homomorphism, it belongs to $G_{0}^{\downarrow}$ if and only if it is $S$-continuous as a mapping $\gamma: G \rightarrow{ }^{*} \mathbb{T}$ with respect to the monadic equivalence $E_{l}=E_{r}$ on $G$ and the usual equivalence of infinitesimal nearness $\approx$ on ${ }^{*} \mathbb{T}$, inherited from the hypercomplex plane ${ }^{*} \mathbb{C}$. The elements of $G_{0}^{\downarrow}$ play the role of finite or accessible characters. The characters $\gamma \in G_{\mathrm{f}}^{\downarrow}$ are infinitesimally close to 1 on the whole subgroup $G_{\mathrm{f}}$ of finite elements of $G$, ie, in front of the horizon they are indistinguishable from the trivial character $1_{G} \in \widehat{G}$. They play the role of infinitesimal characters. However, this intuition calls for some justification.

Let us recall that the Bohr sets $\operatorname{Bohr}_{\alpha}(A), \operatorname{Bohr}_{\alpha}(\Gamma)$ for $A \subseteq G, \Gamma \subseteq \widehat{G}$ and $\alpha \in{ }^{*} \mathbb{R}$, $0 \leq \alpha \leq \pi$, were introduced in Section 2.1.

For $A \subseteq G$ and any subset $T$ of the interval $(0, \pi] \subseteq \mathbb{R}$, such that $\inf T=0$, we obviously have:

$$
A^{\downarrow}=\bigcap_{\alpha \in T} \operatorname{Bohr}_{\alpha}(A)
$$

The point is that for a subgroup $A$ of $G$ a single $\alpha$ suffices.

Lemma 2.2.1 Let $\alpha \in(0,2 \pi / 3)$. If $A$ is a subgroup of $G$ then $A^{\sim}=\operatorname{Bohr}_{\alpha}(A)$.

Proof As each $\gamma \in \widehat{G}$ is a homomorphism, for a subgroup $A \subseteq G$ and $\gamma \in \operatorname{Bohr}_{\alpha}(A)$, the image $\gamma[A]$ must be a subgroup of $* \mathbb{T}$ contained in the $\operatorname{arc}\left\{c \in{ }^{*} \mathbb{T}:|\arg c| \leq \alpha\right\}$. For $\alpha<2 \pi / 3$, however, the biggest subgroup of ${ }^{*} \mathbb{T}$ contained there is namely the monad $\mathbb{I}^{*} \mathbb{T}=\left\{c \in{ }^{*} \mathbb{T}: c \approx 1\right\}$ of $1 \in{ }^{*} \mathbb{T}$. Thus $\gamma[A] \subseteq \mathbb{I}^{*} \mathbb{T}$, hence $\operatorname{Bohr}_{\alpha}(A) \subseteq A^{\downarrow}$, while the reversed inclusion is trivial.

If $A$ is an internal subgroup of $G$ and $0<\alpha<2 \pi / 3$ then a similar argument shows that both the sets coincide with the (strict) annihilator $A^{\perp}$ of $A$; more precisely:

$$
A^{\downarrow}=\operatorname{Bohr}_{\alpha}(A)=\operatorname{Bohr}_{0}(A)=\{\gamma \in \widehat{G}:(\forall a \in A)(\gamma(a)=1)\}=A^{\perp}
$$

Proposition 2.2.2 $\left(\widehat{G}, G_{\mathrm{f}}^{\downarrow}, G_{0}^{\downarrow}\right)$ is a condensing IMG group triplet with a hyperfinite abelian ambient group $\widehat{G}$. 
Proof Let us pick any (standard) $\alpha \in(0,2 \pi / 3)$. As $G_{0}, G_{\mathrm{f}}$ are subgroups of $G$, according to the last lemma we have:

$$
\begin{aligned}
& G_{0}^{\downarrow}=\operatorname{Bohr}_{\alpha}\left(G_{0}\right)=\operatorname{Bohr}_{\alpha}(\bigcap \mathcal{Q})=\bigcup_{Q \in \mathcal{Q}} \operatorname{Bohr}_{\alpha}(Q) \\
& G_{\mathrm{f}}^{\nu}=\operatorname{Bohr}_{\alpha}\left(G_{\mathrm{f}}\right)=\operatorname{Bohr}_{\alpha}(\bigcup \mathcal{Q})=\bigcap_{Q \in \mathcal{Q}} \operatorname{Bohr}_{\alpha}(Q)
\end{aligned}
$$

While the last equation in the second line is trivial, the last equation in the first one follows by a straightforward saturation argument. As all the sets $\operatorname{Bohr}_{\alpha}(Q)$ are internal, $G_{0}^{\downarrow}$ is a galactic set and $G_{\mathrm{f}}^{\downarrow}$ is a monadic one. It remains to show that the IMG group triplet $\left(\widehat{G}, G_{\mathrm{f}}^{\sim}, G_{0}^{\downarrow}\right)$ is condensing.

To this end consider any symmetric internal sets $\Gamma, \Delta$ such that $G_{\mathrm{f}}^{\downarrow} \subseteq \Gamma \subseteq \Delta \subseteq G_{0}^{\downarrow}$. Then there are $P, Q \in \mathcal{Q}$ such that $G_{0} \subseteq P \subseteq Q \subseteq G_{\mathrm{f}}$ and:

$$
G_{\mathrm{f}}^{\downarrow} \subseteq \operatorname{Bohr}_{\alpha}(Q) \subseteq \Gamma \subseteq \Delta \subseteq \operatorname{Bohr}_{\alpha / 4}(P) \subseteq G_{0}^{\downarrow}
$$

Using an elementary combinatorial argument we will show that

$$
\lceil\Delta: \Gamma\rceil \leq\left\lceil\operatorname{Bohr}_{\alpha / 4}(P): \operatorname{Bohr}_{\alpha}(Q)\right\rceil \leq\left\lceil\frac{4 \pi}{\alpha}\right\rceil^{\lfloor Q: P\rfloor}<\infty
$$

where the upper integer part $\lceil 4 \pi / \alpha\rceil$ equals the covering index $\lfloor\mathbb{T}: S\rfloor$ of the circle $\mathbb{T}$ with respect to the $\operatorname{arc} S=\{c \in \mathbb{T}:|\arg c| \leq \alpha / 4\}$. Indeed, let $k=\lfloor\mathbb{T}: S\rfloor$ and $q=\lfloor Q: P\rfloor$. Then there are $k$ points $c_{1}, \ldots, c_{k} \in \mathbb{T}$ and $q$ points $x_{1}, \ldots x_{q} \in G$ such that $\mathbb{T} \subseteq \bigcup_{i=1}^{k} S c_{i}$ and $Q \subseteq \bigcup_{j=1}^{q} P+x_{i}$. Let $h: \mathbb{T} \rightarrow\left\{c_{1}, \ldots, c_{k}\right\}$ be any function such that $c \in S h(c)$ for $c \in \mathbb{T}$. As there are only $k^{q}$ functions $\left\{x_{1}, \ldots, x_{q}\right\} \rightarrow\left\{c_{1}, \ldots, c_{k}\right\}$, given more then $k^{q}$ functions in $\operatorname{Bohr}_{\alpha / 4}(P)$, there are at least two, $\gamma$ and $\chi$, say, such that $(h \circ \gamma)\left(x_{j}\right)=(h \circ \chi)\left(x_{j}\right)$ for $1 \leq j \leq q$. Let's choose any $x \in Q$ and $j \leq q$ such that $x \in P+x_{j}$. Then $\gamma(x) \gamma\left(x_{j}\right)^{-1}, \chi(x) \chi\left(x_{j}\right)^{-1} \in S$ and, as $h\left(\gamma\left(x_{j}\right)\right)=h\left(\chi\left(x_{j}\right)\right)$, we have:

$$
\gamma\left(x_{j}\right) \chi\left(x_{j}\right)^{-1}=\gamma\left(x_{j}\right) h\left(\gamma\left(x_{j}\right)\right)^{-1} h\left(\chi\left(x_{j}\right)\right) \chi\left(x_{j}\right)^{-1} \in S^{2}
$$

Consequently,

$$
\gamma(x) \chi(x)^{-1}=\gamma(x) \gamma\left(x_{j}\right)^{-1} \gamma\left(x_{j}\right) \chi\left(x_{j}\right)^{-1} \chi\left(x_{j}\right) \chi(x)^{-1} \in S^{4}
$$

ie $\gamma \chi^{-1} \in \operatorname{Bohr}_{\alpha}(Q)$. It follows that $\left\lceil\operatorname{Bohr}_{\alpha / 4}(P): \operatorname{Bohr}_{\alpha}(Q)\right\rceil \leq k^{q}$.

The condensing IMG group triplet $\left(\widehat{G}, G_{\mathrm{f}}^{\downarrow}, G_{0}^{\downarrow}\right)$ will be called the dual triplet of the IMG group triplet $\left(G, G_{0}, G_{\mathrm{f}}\right)$. 
For an internal character $\gamma \in G_{0}^{\downarrow}$ there are potentially two interpretations of its observable trace $\gamma^{b}$. First, it is simply the element $G_{\mathrm{f}}^{\downarrow} \gamma$ of the quotient $G_{0}^{\downarrow} / G_{\mathrm{f}}^{\downarrow}=\widehat{G}^{b}$. Second, $\gamma^{b}$ is the observable trace of the $S$-continuous mapping $\gamma: G \rightarrow{ }^{*} \mathbb{T}$, ie

$$
\gamma^{b}\left(x^{b}\right)={ }^{\circ} \gamma(x)
$$

for $x \in G_{\mathrm{f}}$. That way $\gamma^{b}: G^{b} \rightarrow \mathbb{T}$ is a continuous character of the LCA group $G^{b}$ (cf Section 1.3). The assignment $\gamma \mapsto \gamma^{b}$, depicted in the commutative diagram

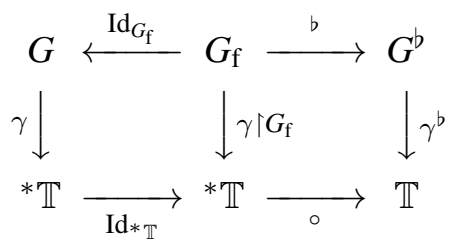

is a group homomorphism $G_{0}^{\downarrow} \rightarrow \widehat{G^{b}}$. Its kernel is the subgroup $G_{\mathrm{f}}^{\downarrow} \subseteq \widehat{G}$ of all infinitesimal characters in $\widehat{G}$. Thus the assignment $\gamma \mapsto \gamma^{b}$ induces an injective group homomorphism $\widehat{G}^{b} \rightarrow \widehat{G^{b}}$ from the observable trace $\widehat{G}^{b}=G_{0}^{\downarrow} / G_{\mathrm{f}}^{\downarrow}$ of the dual triplet $\left(\widehat{G}, G_{\mathrm{f}}^{\downarrow}, G_{0}^{\downarrow}\right)$ into the dual group $\widehat{G^{b}}=\widehat{G_{\mathrm{f}} / G_{0}}$ of the observable trace $G^{b}=G_{\mathrm{f}} / G_{0}$ of the original triplet $\left(G, G_{0}, G_{\mathrm{f}}\right)$. The canonical injective homomorphism $G_{0}^{\nu} / G_{\mathrm{f}}^{\downarrow} \rightarrow \widehat{G_{\mathrm{f}} / G_{0}}$ justifies the identification of the "two observable traces" $G_{\mathrm{f}}^{\downarrow} \gamma$ and $\gamma^{b}$. As proved by Gordon in [17] (see also [18]), even more is true.

Proposition 2.2.3 The canonical mapping $G_{\mathrm{f}}^{\downarrow} \gamma \mapsto \gamma^{b}$ is an isomorphism of the topological group $\widehat{G}^{b}=G_{0}^{\downarrow} / G_{\mathrm{f}}^{\downarrow}$ onto a closed subgroup of the topological group $\widehat{G^{b}}=\widehat{G_{\mathrm{f}} / G_{0}}$.

Proof Denote $\mathbf{G}=G^{b}$ and pick some $\alpha \in(0,2 \pi / 3)$. On the one hand, the images of the Bohr sets $\operatorname{Bohr}_{\alpha}(Q)$, where $Q \in \mathcal{Q}$, under the quotient mapping $G_{0}^{\downarrow} \rightarrow G_{0}^{\downarrow} / G_{\mathrm{f}}^{\downarrow}$ form a neighborhood base of the unit character in $\widehat{G}^{b}$. On the other hand, the Bohr sets

$$
\operatorname{Bohr}_{\alpha}\left(Q^{b}\right)=\left\{\gamma \in \widehat{\mathbf{G}}:\left(\forall \boldsymbol{x} \in Q^{b}\right)(|\arg \gamma(\boldsymbol{x})| \leq \alpha)\right\}
$$

where $Q \in \mathcal{Q}$, form a neighborhood base of the unit character in $\widehat{\mathbf{G}}$. It follows that the canonical injective group homomorphism $G_{0}^{\downarrow} / G_{\mathrm{f}}^{\downarrow} \rightarrow \widehat{\mathbf{G}}$ is also a homeomorphism of $G_{0}^{\downarrow} / G_{\mathrm{f}}^{\downarrow}$ onto the subgroup $\left\{\gamma^{b}: \gamma \in G_{0}^{\downarrow}\right\}$ of $\widehat{\mathbf{G}}$. As a continuous image of an LCA (hence complete) topological group, it is necessarily closed.

It is both natural and tempting to conjecture that the canonical mapping $\widehat{G}^{b} \rightarrow \widehat{G^{b}}$ is also surjective, ie that it is an isomorphism of topological groups. This is indeed the first of Gordon's Conjectures from Gordon [17] (see also [18, page 132]). 
Theorem 2.2.4 (Gordon's Conjecture 1) Let $\left(G, G_{0}, G_{\mathrm{f}}\right)$ be a condensing IMG group triplet with a hyperfinite abelian ambient group $G$. Then the canonical homomorphism $G_{0}^{\downarrow} / G_{\mathrm{f}}^{\downarrow} \rightarrow \widehat{G_{\mathrm{f}} / G_{0}}$ is an isomorphism of topological groups.

The proof of Gordon's Conjecture 1, together with the following Triplet Duality Theorem 2.2.5 which is equivalent to it, is the main result of the present paper. In view of 2.2.3, it amounts just to show that every continuous character $\gamma$ of the LCA group $G^{b}=G_{\mathrm{f}} / G_{0}$ is indeed of the form $\gamma=\gamma^{b}$ for some internal $S$-continuous character $\gamma \in G_{0}^{\downarrow}$. However, we will approach the proof of the above theorem indirectly, by investigating the dual triplet of the dual triplet of the original IMG group triplet $\left(G, G_{0}, G_{\mathrm{f}}\right)$.

The second dual $\widehat{\widehat{G}}$ of the hyperfinite abelian group $G$ can be naturally identified with the original group $G$. Then the second dual of the original triplet $\left(G, G_{0}, G_{\mathrm{f}}\right)$ is defined as the condensing IMG triplet $\left(G, G_{0}^{\downarrow \downarrow}, G_{\mathrm{f}}^{\downarrow \sim}\right)$.

Theorem 2.2.5 (The Triplet Duality Theorem) Let $\left(G, G_{0}, G_{\mathrm{f}}\right)$ be a condensing IMG group triplet with a hyperfinite abelian ambient group $G$. Then:

$$
G_{0}^{\sim \downarrow}=G_{0} \quad \text { and } \quad G_{\mathrm{f}}^{\sim \downarrow}=G_{\mathrm{f}}
$$

In other words, the dual triplet $\left(G, G_{0}^{\downarrow \sim}, G_{\mathrm{f}}^{\downarrow \downarrow}\right)$ of the dual triplet $\left(\widehat{G}, G_{\mathrm{f}}^{\downarrow}, G_{0}^{\downarrow}\right)$ equals the original group triplet $\left(G, G_{0}, G_{\mathrm{f}}\right)$.

The proof of Theorem 2.2.5 is postponed into the next section. At this place we will just show how Gordon's Conjecture 1, ie Theorem 2.2.4, can be derived from Theorem 2.2.5. The proof of this implication relies on the following consequence of the Pontryagin-van Kampen Duality Theorem.

Proposition 2.2.6 Let $\mathbf{G}$ be a Hausdorff LCA group and $\mathbf{D}$ be a subgroup of its dual group $\widehat{\mathbf{G}}$. Then $\mathbf{D}$ separates points in $\mathbf{G}$ if and only if it is dense in $\widehat{\mathbf{G}}$.

Sketch of proof $\widehat{\mathbf{G}}$ obviously separates points in its dual group $\widehat{\widehat{\mathbf{G}}}$ which, according to the Duality Theorem, can be identified with G. Now, it can be easily seen that any dense subgroup $\mathbf{D} \subseteq \widehat{\mathbf{G}}$ separates points in $\mathbf{G}$, as well.

Conversely, if $\mathbf{D}$ is not dense in $\widehat{\mathbf{G}}$ then its closure $\overline{\mathbf{D}}$ is a proper closed subgroup of $\widehat{\mathbf{G}}$. As a consequence of the Duality Theorem (cf Morris [36, Corollary 1 to Theorem 21]), there is a nontrivial character of $\widehat{\mathbf{G}}$, ie, an element $\boldsymbol{x} \in \mathbf{G} \backslash\{0\}$ such that $\gamma(\boldsymbol{x})=1$ for all $\gamma \in \overline{\mathbf{D}}$. Therefore, neither $\overline{\mathbf{D}}$ nor $\mathbf{D}$ separate points in $\mathbf{G}$. 
Proof of 2.2.5 $\Rightarrow$ 2.2.4 By Proposition 2.2.3, the observable trace $\widehat{G}^{b}=G_{0}^{\downarrow} / G_{\mathrm{f}}^{\downarrow}$ of the dual triplet $\left(\widehat{G}, G_{\mathrm{f}}^{\downarrow}, G_{0}^{\downarrow}\right)$ can be identified with the closed subgroup of the dual group $\widehat{G^{b}}$ formed by the observable traces $\gamma^{b}$ of the internal characters $\gamma \in G_{0}^{\downarrow}$. Therefore, in order to show that $\widehat{G}^{b}=\widehat{G^{b}}$, it suffices to prove that $\widehat{G}^{b}$ is dense in $\widehat{G^{b}}$. By Proposition 2.2.6, to this end it is enough to show that $\widehat{G}^{b}$ separates points in $G^{b}$. But otherwise there would be an $x \in G_{\mathrm{f}} \backslash G_{0}$, such that $\gamma(x) \approx 1$ for every $\gamma \in G_{0}^{\downarrow}$. Then

$$
x \in G_{\mathrm{f}} \cap G_{0}^{\downarrow \downarrow}=G_{\mathrm{f}} \cap G_{0}=G_{0}
$$

which is a contradiction.

Remark Notice that, in order to derive 2.2.4, just a weaker version of the first equality in 2.2.5 would be sufficient, namely $G_{0}=G_{0}^{\downarrow \downarrow} \cap G_{\mathrm{f}}$, which, of course, trivially follows from $G_{0}^{\downarrow \downarrow}=G_{0}$ and $G_{0} \subseteq G_{\mathrm{f}}$. The second equality $G_{\mathrm{f}}^{\downarrow \downarrow}=G_{\mathrm{f}}$ is not needed to this end. Moreover, it is already a consequence of the first one.

Lemma 2.2.7 Let $\left(G, G_{0}, G_{\mathrm{f}}\right)$ be as above. Then:

$$
G_{\mathrm{f}}^{\downarrow \downarrow}=G_{\mathrm{f}}+G_{0}^{\downarrow \downarrow}
$$

Therefore, $G_{0}^{\downarrow \downarrow}=G_{0}$ implies $G_{\mathrm{f}}^{\downarrow \downarrow}=G_{\mathrm{f}}$.

Proof Let us denote $\left(H, H_{0}, H_{\mathrm{f}}\right)=\left(\widehat{G}, G_{\mathrm{f}}^{\downarrow}, G_{0}^{\downarrow}\right)$, the dual triplet of $\left(G, G_{0}, G_{\mathrm{f}}\right)$. According to the properties of Galois connections, three $\mathcal{L}$ 's reduce to one, hence the group triplet $\left(H, H_{0}, H_{\mathrm{f}}\right)$ satisfies the conditions

$$
H_{0}^{\downarrow \sim}=H_{0} \quad \text { and } \quad H_{\mathrm{f}}^{\downarrow \nu}=H_{\mathrm{f}}
$$

so that the dual triplet of $\left(\widehat{H}, H_{\mathrm{f}}^{\downarrow}, H_{0}^{\downarrow}\right)$ is indeed $\left(H, H_{0}, H_{\mathrm{f}}\right)$ and the canonical mapping $H_{0}^{\downarrow} / H_{\mathrm{f}}^{\downarrow} \rightarrow \widehat{H_{\mathrm{f}} / H_{0}}$ is an isomorphism of topological groups.

Now, consider the canonical embedding $G_{0}^{\downarrow} / G_{\mathrm{f}}^{\downarrow} \rightarrow \widehat{G_{\mathrm{f}} / G_{0}}$ and apply the (external) duality functor to it. Identifying the dual of the LCA group $\widehat{G_{\mathrm{f}} / G_{0}}$ with $G_{\mathrm{f}} / G_{0}$ and the dual of the LCA group $H_{\mathrm{f}} / H_{0}=G_{0}^{\sim} / G_{\mathrm{f}}^{\downarrow}$ with $H_{0}^{\sim} / H_{\mathrm{f}}^{\sim}=G_{\mathrm{f}}^{\sim} / G_{0}^{\sim}$, we obtain a surjective continuous homomorphism

$$
G_{\mathrm{f}} / G_{0} \rightarrow G_{\mathrm{f}}^{\downarrow \nu} / G_{0}^{\downarrow \nu}
$$

sending $\gamma+G_{0} \in G_{\mathrm{f}} / G_{0}$ to $\gamma+G_{0}^{\downarrow \nu} \in G_{\mathrm{f}}^{\sim \downarrow} / G_{0}^{\downarrow \nu}$. Its surjectivity simply means that $G_{\mathrm{f}}, G_{0}^{\downarrow \sim}$ and $G_{\mathrm{f}}^{\sim} \sim$, as subgroups of $G$, satisfy $G_{\mathrm{f}}^{\sim \sim}=G_{\mathrm{f}}+G_{0}^{\downarrow \nu}$. 
The following example shows that (a) the condition $X^{\downarrow \downarrow}=X$ is not automatically satisfied for monadic or galactic subgroups $X$ of hyperfinite abelian groups, and (b) there are plenty of subgroups of hyperfinite abelian groups satisfying this condition even without being monadic or galactic.

Example 2.2.8 An additive cut on ${ }^{*} \mathbb{N}$ is any nonempty subset $C \subseteq{ }^{*} \mathbb{N}$, such that:

$$
\begin{gathered}
\left(\forall a \in{ }^{*} \mathbb{N}\right)(\forall b \in C)(a \leq b \Rightarrow a \in C) \\
(\forall a, b \in C)(a+b \in C)
\end{gathered}
$$

Assume that $C$ is an additive cut on ${ }^{*} \mathbb{N}, \mathbb{N} \subseteq C$, and $n \in{ }^{*} \mathbb{N} \backslash C$.

(a) Let $Z$ be any nontrivial finite abelian group, eg $Z=\mathbb{Z}_{d}$ for some $2 \leq d \in \mathbb{N}$. Let $G=Z^{n}$ be the hyperfinite abelian group of all internal sequences $z=\left(z_{1}, \ldots, z_{n}\right)$ of elements from $Z$. Denote

$$
\operatorname{supp} z=\left\{k: 1 \leq k \leq n \& z_{k} \neq 0\right\}
$$

for $z \in G$, and put:

$$
S(C)=\{z \in G:|\operatorname{supp} z| \in C\}
$$

As $C \neq\{0\}$ and $n \notin C$, as well as $\operatorname{supp}(-z)=\operatorname{supp} z$ and $\operatorname{supp}(y+z) \subseteq \operatorname{supp} y \cup \operatorname{supp} z$ for any $y, z \in G, S(C)$ is a nontrivial proper subgroup of $G$. Moreover, if $C$ is a monadic (galactic) set then so is $S(C)$. On the other hand, all the internal subgroups

$$
G_{k}=\{z \in G: \operatorname{supp} z \subseteq\{k\}\}=\left\{z \in G:(\forall i \neq k)\left(z_{i}=0\right)\right\} \cong Z
$$

where $1 \leq k \leq n$, satisfy $G_{k} \subseteq S(C)$. Identifying the internal dual $\widehat{G}$ with the hyperfinite abelian group $\widehat{Z}^{n}$ in the obvious way, each character $\gamma \in \widehat{G}$ is represented as the ordered $n$-tuple $\gamma=\left(\gamma_{1}, \ldots, \gamma_{n}\right)$, where $\gamma_{k} \in \widehat{Z}$. Then

$$
G_{k}^{\downarrow}=\left\{\gamma \in \widehat{G}: \gamma_{k}=1_{Z}\right\}
$$

hence

$$
S(C)^{\sim} \subseteq \bigcap_{k=1}^{n} G_{k}^{\nu}=\left\{1_{G}\right\}
$$

and, finally, $S(C)^{\downarrow \sim}=G$.

Now, if we take a monadic additive cut $A$ and a galactic additive cut $B$, such that $\mathbb{N} \subseteq A \subseteq B \subseteq{ }^{*} \mathbb{N}$ and $n \notin B$, then $S(A)$ is a proper subgroup of $S(B)$ and $(G, S(A), S(B))$ is an (of course, non-condensing) IMG group triplet with a hyperfinite abelian ambient group $G=Z^{n}$ and nontrivial observable trace $S(B) / S(A)$. However, its first and second dual triplets are $\left(\widehat{G},\left\{1_{G}\right\},\left\{1_{G}\right\}\right)$ and $(G, G, G)$, respectively; both have trivial observable traces. 
(b) Denote $A=C \cup(-C)=\left\{a \in{ }^{*} \mathbb{Z}:|a| \in C\right\}$. Then $A$ is a subgroup of the hyperfinite cyclic group $\mathbb{Z}_{2 n+1}=\{0, \pm 1, \ldots, \pm n\}$ of remainders modulo $2 n+1$. We leave the reader to verify that $A^{\sim} \sim=A$, regardless of any further properties of $C$.

\subsection{The dual triplet continued: normalizing multipliers and the proofs of Gordon's Conjectures 1 and 2}

Let us recall that $\left(G, G_{0}, G_{\mathrm{f}}\right)$ still denotes some condensing IMG group triplet with a hyperfinite abelian ambient group $G$. A normalizing multiplier of this triplet is any positive hyperreal number $d$ such that $0 \not \approx d|A|<\infty$ for every (or, equivalently, for some) internal set $A$ between $G_{0}$ and $G_{\mathrm{f}}$. Then $\boldsymbol{m}_{d}$ denotes the Haar measure on the observable trace $G^{b}=G_{\mathrm{f}} / G_{0}$, obtained by pushing down the Loeb measure $\lambda_{d}$ from $G$ to $G^{b}$ (see Sections 1.2 and 1.3). The Hermitian inner product, the Fourier transform, the $\mathrm{L}^{p}$-norms and the convolution on the space ${ }^{*} \mathbb{C}^{G}$ of all internal functions $G \rightarrow{ }^{*} \mathbb{C}$ are normalized by the normalizing coefficient $d$, ie

$$
\begin{gathered}
\langle f, g\rangle=d \sum_{x \in G} f(x) \bar{g}(x) \\
\mathcal{F}(f)(\gamma)=\widehat{f}(\gamma)=\langle f, \gamma\rangle=d \sum_{x \in G} f(x) \bar{\gamma}(x) \\
\|f\|_{p}=\left(d \sum_{x \in G}|f(x)|^{p}\right)^{1 / p} \\
(f * g)(x)=d \sum_{a \in G} f(x-a) g(a)=d \sum_{a \in G} f_{a}(x) g(a)
\end{gathered}
$$

for $f, g \in \mathbb{C}^{G}, \gamma \in \widehat{G}, 1 \leq p<\infty, x \in G$.

In the proof of the Triplet Duality Theorem 2.2.5 we will need the following preliminary qualitative version of the Smoothness-and-Decay Principle.

Proposition 2.3.1 Let $f: G \rightarrow{ }^{*} \mathbb{C}$ be an $S$-continuous internal function such that $\operatorname{supp} f \subseteq G_{\mathrm{f}}$. Then $\widehat{f}(\gamma) \approx 0$ for each $\gamma \in \widehat{G} \backslash G_{0}^{\downarrow}$.

Proof We will proceed in a similar way as in the proof of Proposition 2.1.5.

By the $S$-continuity of $f, f_{a}(x) \approx f(x)$ for each $x \in G$, whenever $a \in G_{0}$; moreover $f(x)=f_{a}(x)=0$ once $x \in G \backslash D$ for some (in fact for each) internal set $D$ such that $\operatorname{supp} f+G_{0} \subseteq D \subseteq G_{\mathrm{f}}$. Then

$$
\left\|\widehat{f_{a}}-\widehat{f}\right\|_{\infty} \leq\left\|f_{a}-f\right\|_{1}=d \sum_{x \in D}\left|f_{a}(x)-f(x)\right| \leq d|D| \max _{x \in D}\left|f_{a}(x)-f(x)\right| \approx 0
$$


as $d|D|<\infty$. Now, for any $\gamma \in \widehat{G} \backslash G_{0}^{\downarrow}$ there is some $a \in G_{0}$ such that $\gamma(a) \not \approx 1$. Then:

$$
0 \approx\left\|\widehat{f_{a}}-\widehat{f}\right\|_{\infty}=\max _{\chi \in \widehat{G}}|\bar{\chi}(a)-1||\widehat{f}(\chi)| \geq|\gamma(a)-1||\widehat{f}(\gamma)|
$$

As $\gamma(a)-1 \not \approx 0$, the conclusion $\widehat{f}(\gamma) \approx 0$ follows immediately.

Corollary 2.3.2 Let $f: G \rightarrow{ }^{*} \mathbb{C}$ be an $S$-continuous internal function such that $\operatorname{supp} f \subseteq G_{\mathrm{f}}$ and $\|f\|_{1} \not \approx 0$. Assume that $t \in{ }^{*} \mathbb{R}$ and $0<t \leq 1, t \not \approx 0$. Then $\operatorname{Spec}_{t}(f) \subseteq G_{0}^{\downarrow}$.

Remark Both Proposition 2.3.1 and its Corollary 2.3.2, are "soft" statements in the sense of Tao's blog which appeared in his book [43, § 1.3, § 1.5]. Their "hard" version is Proposition 2.1.5 and its Corollary 2.1.6, providing us with the additional information how one has to choose $\varepsilon>0$ and the internal set $C$, such that $G_{0} \subseteq C \subseteq G_{\mathrm{f}}$, in order to get $\operatorname{Spec}_{t}(f) \subseteq \operatorname{Bohr}_{\alpha}(C) \subseteq G_{0}^{\downarrow}$. On the other hand, the last corollary is fully sufficient for our purpose. We hope that at least some readers will appreciate the advantage of nonstandard arguments, allowing one to dispense with lots of meticulous estimates and "epsilontics".

As made clear in Section 2.2, in order to finish the proof of the Triplet Duality Theorem 2.2.5, as well as of Gordon's Conjecture 1 (Theorem 2.2.4), it is enough to prove the following:

Proposition 2.3.3 Let $\left(G, G_{0}, G_{\mathrm{f}}\right)$ be a condensing IMG group triplet with hyperfinite abelian ambient group $G$. Then:

$$
G_{0}^{\downarrow \sim}=G_{0}
$$

Proof Since $G_{0} \subseteq G_{0}^{\downarrow \downarrow}$ is obvious, it suffices to prove the opposite inclusion. Let $\mathcal{V}$ be a set of internal gauges on $G$ such that

$$
G_{0}=\{x \in G:(\forall \varrho \in \mathcal{V})(\varrho(x) \approx 0)\}
$$

whose existence was established in Proposition 1.3.1. For any $\varrho \in \mathcal{V}$ and a positive $r \in{ }^{*} \mathbb{R}$ we denote by

$$
B_{\varrho}(r)=\{x \in G: \varrho(x) \leq r\}
$$

the internal closed ball of radius $r$, and define the internal function $h_{\varrho r}: G \rightarrow{ }^{*} \mathbb{R}$ by:

$$
h_{\varrho r}(x)=\max \left\{1-\frac{\varrho(x)}{r}, 0\right\}
$$

Journal of Logic \& Analysis 13:1 (2021) 
Obviously, $h_{\varrho r}$ is even, nonnegative, and $\operatorname{supp} h_{\varrho r} \subseteq B_{\varrho}(r)$. For $0 \not \approx r<$, both the sets supp $h_{\varrho}$ and $B_{\varrho}(r)$ are between $G_{0}$ and $G_{\mathrm{f}}$. Moreover, for $r$ noninfinitesimal, $h_{\varrho}$ is also $S$-continuous and $\left\|h_{\varrho r}\right\|_{1} \geq d\left|B_{\varrho}(r / 2)\right| / 2 \not \approx 0$. By Corollary 2.3.2,

$$
\operatorname{Spec}_{t}\left(h_{\varrho r}\right) \subseteq G_{0}^{\downarrow}
$$

for $0<t \leq 1$, once $t$ is noninfinitesimal, too. Further on, $\operatorname{supp}\left(h_{\varrho} * h_{\varrho}\right) \subseteq B_{\varrho}(2 r)$. In order to apply Proposition 2.2.3 we need the estimate:

$$
\frac{\left\|h_{\varrho r}\right\|_{1}}{\left\|h_{\varrho r}\right\|_{2}\left\|1_{B_{\varrho}(2 r)}\right\|_{2}} \geq \frac{\frac{1}{2}\left|B_{\varrho}(r / 2)\right|}{\sqrt{\left|B_{\varrho}(r)\right|\left|B_{\varrho}(2 r)\right|}} \geq \frac{\left|B_{\varrho}(r / 2)\right|}{2\left|B_{\varrho}(2 r)\right|} \not 0
$$

Thus picking some standard positive $\alpha<\pi / 2$, there is a standard $t>0$ with:

$$
t \leq \frac{\left\|h_{\varrho r}\right\|_{1}}{\left\|h_{\varrho r}\right\|_{2}\left\|1_{B_{\varrho}(2 r)}\right\|_{2}} \cdot \sqrt{\frac{\cos \alpha}{1+\cos \alpha}}
$$

For such $\alpha$ and $t$ we have:

$$
\operatorname{Bohr}_{\alpha}\left(\operatorname{Spec}_{t}\left(h_{\varrho}\right)\right) \subseteq B_{\varrho}(2 r)-B_{\varrho}(2 r) \subseteq B_{\varrho}(4 r)
$$

Consequently:

$$
G_{0}^{\downarrow \sim}=\operatorname{Bohr}_{\alpha}\left(G_{0}^{\downarrow}\right) \subseteq \operatorname{Bohr}_{\alpha}\left(\operatorname{Spec}_{t}\left(h_{\varrho} r\right)\right) \subseteq B_{\varrho}(4 r)
$$

As $\varrho$ and $r$ were arbitrary, this is enough to establish the inclusion $G_{0}^{\downarrow \downarrow} \subseteq G_{0}$.

Remark In fact the subgroup $G_{\mathrm{f}}$ plays no role in the above statement nor in its proof. The only thing we need to assume is the existence of an internal set $A$ subject to $G_{0} \subseteq A \subseteq G$ such that $|A| /|B|<\infty$ for each internal $B$ such that $G_{0} \subseteq B \subseteq A$.

Having $d$ as a normalizing multiplier for the triplet $\left(G, G_{0}, G_{\mathrm{f}}\right)$, then, in order to get the Fourier inversion formula and Plancherel identity, we need to normalize the inner product, the Fourier transform, etc, on the space ${ }^{*} \mathbb{C}^{\widehat{G}}$ of internal functions $\widehat{G} \rightarrow{ }^{*} \mathbb{C}$, defined on the internal dual group $\widehat{G}$, by means of the normalizing coefficient:

$$
\widehat{d}=\frac{1}{d|G|}
$$

In view of the canonical isomorphism of the observable trace $\widehat{G}^{b}=G_{0}^{\downarrow} / G_{\mathrm{f}}^{\downarrow}$ and the dual group $\widehat{G^{b}}$, there naturally arises the question whether such a $\widehat{d}$ is a normalizing multiplier for the dual triplet $\left(\widehat{G}, G_{\mathrm{f}}^{\downarrow}, G_{0}^{\downarrow}\right)$. In that case (and only in that case) the measure $\boldsymbol{m}_{\widehat{d}}$, obtained by pushing down the Loeb measure $\lambda_{\widehat{d}}$ from $\widehat{G}$ to the observable trace $\widehat{G}^{b} \cong \widehat{G^{b}}$, will be a Haar measure on $\widehat{G}^{b}$. The second of Gordon's conjectures, whose proof is the second main result of this paper, states that the response to the above question is affirmative. 
Theorem 2.3.4 (Gordon's Conjecture 2) Let $\left(G, G_{0}, G_{\mathrm{f}}\right)$ be a condensing IMG group triplet with a hyperfinite abelian ambient group $G$. Then, for any internal set $D$ such that $G_{0} \subseteq D \subseteq G_{\mathrm{f}}, d=1 /|D|$ is a normalizing multiplier for $\left(G, G_{0}, G_{\mathrm{f}}\right)$ and $\widehat{d}=|D| /|G|$ is a normalizing multiplier for the dual triplet $\left(\widehat{G}, G_{\mathrm{f}}^{\downarrow}, G_{0}^{\downarrow}\right)$. More generally, if $d$ is any normalizing multiplier for $\left(G, G_{0}, G_{\mathrm{f}}\right)$ then $\widehat{d}=(d|G|)^{-1}$ is a normalizing multiplier for $\left(\widehat{G}, G_{\mathrm{f}}^{\downarrow}, G_{0}^{\downarrow}\right)$.

Proof Since, for any internal set $D$ such that $G_{0} \subseteq D \subseteq G_{\mathrm{f}}, d=1 /|D|$ is a normalizing multiplier for the triplet $\left(G, G_{0}, G_{\mathrm{f}}\right)$ and $d_{1} / d_{2} \in \mathbb{F}^{*} \mathbb{R} \backslash \mathbb{I}^{*} \mathbb{R}$ for any its normalizing multipliers $d_{1}, d_{2}$, it suffices to find a single couple of internal sets $D$ between $G_{0}, G_{\mathrm{f}}$ and $\Gamma$ between $G_{\mathrm{f}}^{\downarrow}, G_{0}^{\downarrow}$, such that the quotient $|D||\Gamma| /|G|$ is neither infinite nor infinitesimal.

Starting with any internal gauge $\varrho \in \mathcal{V}$, we have $G_{0} \subseteq B_{\varrho}(r) \subseteq G_{\mathrm{f}}$ whenever $0<r \in \mathbb{R}$. Also, we can take arbitrary standard $\alpha$ and $t$ subject to $0<\alpha<\pi / 2$, $0<t \leq \cos \alpha$. Then the spectral set $\Gamma=\operatorname{Spec}_{t}\left(h_{\varrho r}\right)$ is internal and, by Lemma 2.1.2 and Corollary 2.3.2, it satisfies the inclusions:

$$
G_{\mathrm{f}}^{\downarrow} \subseteq \operatorname{Bohr}_{\alpha}\left(B_{\varrho}(r)\right) \subseteq \operatorname{Spec}_{t}\left(h_{\varrho}\right) \subseteq G_{0}^{\downarrow}
$$

Denoting $f=h_{\varrho r}, D=B_{\varrho}(2 r)$, we have $\operatorname{supp}(f * f) \subseteq D \subseteq G_{\mathrm{f}}$, and multiplying the inequalities in Proposition 2.1.4 by the factor $\left\|1_{D}\right\|_{2}^{2}=|D| /|G|$ we get:

$$
1-t^{2} \frac{\|f\|_{2}^{2}\left\|1_{D}\right\|_{2}^{2}}{\|f\|_{1}^{2}} \leq \frac{|D|\left|\operatorname{Spec}_{t}(f)\right|}{|G|} \leq \frac{1}{t^{2}} \frac{\|f\|_{2}^{2}\left\|1_{D}\right\|_{2}^{2}}{\|f\|_{1}^{2}}
$$

Since

$$
\frac{\|f\|_{2}^{2}\left\|1_{D}\right\|_{2}^{2}}{\|f\|_{1}^{2}} \leq \frac{\left|B_{\varrho}(r)\right|\left|B_{\varrho}(2 r)\right|}{\left(\frac{1}{2}\left|B_{\varrho}(r / 2)\right|\right)^{2}}<\infty
$$

the upper bound in the preceding inequality is finite for any standard $t>0$. For the same reason, it is possible to find a standard $t$, such that $0<t \leq \cos \alpha$, and making the lower bound positive and noninfinitesimal.

\subsection{Some (mainly) standard equivalents: Hrushovski style theorems}

In this section we list some direct standard consequences (in fact, equivalents) of Theorem 2.2.4 and Proposition 2.3.3. The formulations of Theorems 2.4.2 and 2.4.3 below remind of the formulation of Hrushovski's structure theorem [26, Theorem 1.1, Corollaries 4.13 and 4.15] (see also Breuillard, Green and Tao [5, Theorem 6.18]). As both the implications 2.2.4 $\Rightarrow 2.4 .1$ and 2.3.3 $\Rightarrow 2.4 .3$ can be proved using a similar 
way of argumentation, we give just the proof of the former (which is technically more complicated), introducing an additional nonstandard equivalent formulated as Theorem 2.4.1.

As we will show within short, Gordon's Conjecture 1 (Theorem 2.2.4) is equivalent to a kind of almost-near or stability principle for characters of hyperfinite abelian groups. The following notions are needed for the nonstandard formulation of this principle.

Let $G$ be an internal abelian group and $X$ be a (not necessarily internal) subset of $G$. Two internal mappings $f: C \rightarrow{ }^{*} \mathbb{T}, g: D \rightarrow{ }^{*} \mathbb{T}$, defined on sets $C, D \subseteq G$, are said to be infinitesimally close on a set $X \subseteq C \cap D$ if $f(x) \approx g(x)$ for all $x \in X$. We say that $g$ is almost homomorphic on a set $X$ if $X \subseteq D$ and, for all $x, y \in X, x+y \in X$ implies:

$$
g(x+y) \approx g(x) g(y)
$$

Theorem 2.4.1 Let $\left(G, G_{0}, G_{\mathrm{f}}\right)$ be a condensing IMG group triplet with a hyperfinite abelian ambient group $G$, and $g: D \rightarrow{ }^{*} \mathbb{T}$ be an internal mapping, where $G_{\mathrm{f}} \subseteq D \subseteq G$. If $g$ is $S$-continuous and almost homomorphic on $G_{\mathrm{f}}$ then there exists an internal $S$-continuous character $\gamma: G \rightarrow{ }^{*} \mathbb{T}$, ie $\gamma \in G_{0}^{\downarrow}$, such that $g$ and $\gamma$ are infinitesimally close on $G_{\mathrm{f}}$.

Due to saturation, any internal mapping $g: D \rightarrow{ }^{*} \mathbb{T}$, where $G_{\mathrm{f}} \subseteq D \subseteq G$, which is almost homomorphic on $G_{\mathrm{f}}$, must be almost homomorphic on some symmetric internal set $C$ such that $G_{\mathrm{f}} \subseteq C \subseteq D$. Similarly, if $g$ and $\gamma$ are infinitesimally close on $G_{\mathrm{f}}$ then they must be infinitesimally close on some symmetric internal set between $G_{\mathrm{f}}$ and $D$. At the same time, internal mappings $g: D \rightarrow{ }^{*} \mathbb{T}$ almost homomorphic on the galaxy $G_{\mathrm{f}}$ share the following property with internal homomorphisms $\gamma \in \widehat{G}$ : such a $g$ is $S$-continuous on $G_{\mathrm{f}}$ if and only if, given any (standard) $\alpha \in(0,2 \pi / 3)$, there is some internal set $A$ between $G_{0}$ and $G_{\mathrm{f}}$ such that $|\arg g(x)| \leq \alpha$ for all $x \in A$ (cf Lemma 2.2.1 and the beginning of the proof of Proposition 2.2.2).

The reformulation of Theorem 2.4.1 in standard terms is a highly uniform, but rather cumbersome stability principle for characters of finite abelian groups. For the sake of its formulation, as well as of the proof of the equivalence of Theorems 2.2.4, 2.4.1 and 2.4.2 (that way proving Theorems 2.4.1 and 2.4.2, as well), we have to introduce some further notions.

Let $G$ be an abelian group, and $\varepsilon>0$ be a (standard) real. Two mappings $f: C \rightarrow \mathbb{T}$, $g: D \rightarrow \mathbb{T}$, defined on subsets $C, D \subseteq G$, are said to be $\varepsilon$-close on the set $X \subseteq C \cap D$ if

$$
\left|\arg \frac{f(x)}{g(x)}\right| \leq \varepsilon
$$


for all $x \in X$. We say that $g$ is $\varepsilon$-homomorphic on the set $X \subseteq D$ if for all $x, y \in X$ the condition $x+y \in X$ implies:

$$
\left|\arg \frac{g(x) g(y)}{g(x+y)}\right| \leq \varepsilon
$$

Finally, $g$ is called a partial $\varepsilon$-homomorphism if it is $\varepsilon$-homomorphic on its domain $D$.

In what follows the expression $[A: B]$ denotes any of the indices $\lfloor A: B\rfloor,\lfloor A: B\rfloor_{\mathrm{i}}$, $\lceil A: B\rceil$, introduced in Section 1.2, or the quotient $|A| / \mid B]$ (see also Proposition 1.3.5).

Theorem 2.4.2 Let $\alpha, \varepsilon \in(0,2 \pi / 3), k \geq 1$ and $\left(q_{j}\right)_{j=1}^{\infty}$ be any sequence of reals $q_{j} \geq 1$. Then there exist $m \geq 1, n \geq k$ and $\delta>0$, depending just on $\alpha, \varepsilon, k$ and the sequence $\left(q_{j}\right)$, such that the following holds:

Let $G$ be a finite abelian group and $0 \in A_{n} \subseteq \ldots \subseteq A_{1} \subseteq A_{0} \subseteq G$ be symmetric sets such that

$$
A_{j}+A_{j} \subseteq A_{j-1} \quad \text { and } \quad\left[A_{j-1}: A_{j}\right] \leq q_{j}
$$

for $1 \leq j \leq n$. Then, for every partial $\delta$-homomorphism $g: m A_{0} \rightarrow \mathbb{T}$ such that $|\arg g(x)| \leq \alpha$ for $x \in A_{k}$, there exists a homomorphism $\gamma: G \rightarrow \mathbb{T}$ such that $g$ and $\gamma$ are $\varepsilon$-close on $A_{0}$.

Proof of 2.2.4 $\Rightarrow$ 2.4.2 Assume that 2.2.4 holds and 2.4.2 is not true. Then we can fix some $\alpha, \varepsilon, k$ and a sequence $\left(q_{j}\right)$ witnessing a counterexample. Let $\left(\delta_{n}\right)$ be any strictly decreasing sequence such that $\delta_{n} \rightarrow 0$. Then for all $m=n \geq k$ there exist a finite abelian group $G_{n}$ and symmetric sets $0 \in A_{n n} \subseteq \ldots \subseteq A_{n 1} \subseteq A_{n 0} \subseteq G_{n}$ such that

$$
A_{n j}+A_{n j} \subseteq A_{n j-1} \quad \text { and } \quad\left[A_{n j-1}: A_{n j}\right] \leq q_{j}
$$

for $1 \leq j \leq n$, as well as a partial $\delta_{n}$-homomorphism $g_{n}: n A_{n 0} \rightarrow \mathbb{T}$ such that $\left|\arg g_{n}(x)\right| \leq \alpha$ for $x \in A_{n k}$. On the other hand, for every genuine homomorphism $\gamma_{n}: G_{n} \rightarrow \mathbb{T}$, there is $x_{n} \in A_{n 0}$ such that:

$$
\left|\arg \frac{\gamma_{n}\left(x_{n}\right)}{g_{n}\left(x_{n}\right)}\right|>\varepsilon
$$

Let $\mathcal{D}$ be any nonprincipal ultrafilter on the set $I=\{n \in \mathbb{N}: n \geq k\}$. Then the ultraproduct $G=\prod_{n \in I} G_{n} / \mathcal{D}$ of the finite groups $G_{n}$ is a hyperfinite abelian group (within the $\aleph_{1}$-saturated nonstandard universe obtained via the ultraproduct construction modulo $\mathcal{D}$ ). For each $n \in I, n<j$, we put $A_{n j}=\{0\}$ and take the ultraproduct $A_{j}=\prod_{n \in I} A_{n j} / \mathcal{D}$ considered as an internal subset of $G$. Finally, we put:

$$
G_{0}=\bigcap_{j \in \mathbb{N}} A_{j} \quad G_{\mathrm{f}}=\bigcup_{j \in \mathbb{N}} j A_{0}
$$


Then $G_{0}$, as an intersection of countably many internal sets, is a monadic subgroup of $G$ and $G_{\mathrm{f}}$, as a union of countably many internal sets, is a galactic subgroup of $G$. Obviously, $G_{0} \subseteq G_{\mathrm{f}}$. By Łos' Theorem, $\left[A_{j-1}: A_{j}\right] \leq q_{j}$, as well as $\left[j A_{0}: A_{1}\right] \leq q_{1}^{j}$ for each $j \geq 1$. It follows easily that $[V: U]<\infty$ for any internal sets $U, V$ such that $G_{0} \subseteq U \subseteq V \subseteq G_{\mathrm{f}}$, hence, by Proposition 1.3.5, $\left(G, G_{0}, G_{\mathrm{f}}\right)$ is a condensing IMG triplet with a hyperfinite abelian ambient group $G$.

Let us form the internal mapping $g=\left(g_{n}\right)_{n \in I} / \mathcal{D}$. Then, for each $n, g$ is a partial $\delta_{n}$-homomorphisms from the internal set $\prod_{n \in I} n A_{n 0} / \mathcal{D} \supseteq G_{\mathrm{f}}$ to the ultrapower group ${ }^{*} \mathbb{T}=\mathbb{T}^{I} / \mathcal{D}$. Thus $g$ is almost homomorphic on $G_{\mathrm{f}}$ and it maps the internal set $A_{k}=\prod_{n} A_{n k} / \mathcal{D}$, satisfying $G_{0} \subseteq A_{k} \subseteq G_{\mathrm{f}}$, into the $\operatorname{arc}\left\{c \in{ }^{*} \mathbb{T}:|\arg c| \leq \alpha\right\}$. It follows that $g$ is $S$-continuous on $G_{\mathrm{f}}$. Then the observable trace $g^{b}={ }^{\circ}\left(g\left\lceil G_{\mathrm{f}}\right)\right.$ is a continuous character of the LCA group $G^{b}=G_{\mathrm{f}} / G_{0}$. By Theorem 2.2.4, there is an internal character $\gamma=\left(\gamma_{n}\right) / \mathcal{D} \in G_{0}^{\downarrow}$ such that $g(x) \approx \gamma(x)$ for each $x \in G_{\mathrm{f}}$. On the other hand, there is a set $J \in \mathcal{D}$ such that $\gamma_{n} \in \widehat{G}_{n}$ for all $n \in J$. By our assumptions, for each $n \in J$, there is $x_{n} \in A_{n 0}$ such that $\left|\arg \left(\gamma_{n}\left(x_{n}\right) / g_{n}\left(x_{n}\right)\right)\right|>\varepsilon$. Let $x_{n}=0$ for $n \in I \backslash J$. Then, for $x=\left(x_{n}\right) / \mathcal{D} \in A_{0} \subseteq G_{\mathrm{f}}$, we have $g(x) \not \approx \gamma(x)$, a contradiction.

Remark Concerning $m, n$ the result is purely existential, giving no upper bound for them (though we can always have $m=n$ ). On the other hand, it seems rather probable that even a more uniform version of Theorem 2.4.2 is true. We conjecture that, similar to Mačaj and Zlatoš [33, Theorem 4.1], one can take any $\delta>0$, such that $\delta<\min \{\varepsilon, \pi / 2,2 \pi / 3-\alpha\}$, and choose $m, n$ depending additionally on $\delta$.

Proof of 2.4.2 $\Rightarrow$ 2.4.1 Let $\left(G, G_{0}, G_{\mathrm{f}}\right)$ be a condensing IMG group triplet with a hyperfinite abelian ambient group $G, D$ be an internal set such that $G_{\mathrm{f}} \subseteq D \subseteq G$ and $g: D \rightarrow{ }^{*} \mathbb{T}$ be an internal mapping that is $S$-continuous and almost homomorphic on $G_{\mathrm{f}}$. We can also assume that $g(0)=1$. Thus fixing a standard $\alpha \in(0,2 \pi / 3)$, there is a symmetric internal set $V$ between $G_{0}$ and $G_{\mathrm{f}}$ such that $|\arg g(x)| \leq \alpha$ for $x \in V$. Let us choose, additionally, a $\beta \in(\alpha, 2 \pi / 3)$.

It suffices to show that, for each symmetric internal set $A$ such that $V+V \subseteq A \subseteq G_{\mathrm{f}}$, and each standard $\varepsilon>0$, such that $\alpha+\varepsilon \leq \beta$, there is $\gamma \in \operatorname{Bohr}_{\beta}(V)$ such that

$$
\left|\arg \frac{g(x)}{\gamma(x)}\right| \leq \varepsilon
$$

for all $x \in A$. Then, due to saturation, there is $\gamma \in \operatorname{Bohr}_{\beta}(V) \subseteq G_{0}^{\downarrow}$ such that $\gamma(x) \approx g(x)$ for all $x \in G_{\mathrm{f}}$, ie, $\gamma$ and $g$ are infinitesimally close on $G_{\mathrm{f}}$.

So let us fix some $A, V, \alpha, \beta$ and $\varepsilon$, satisfying the above assumptions. There is a sequence of symmetric internal sets $\left(A_{j}\right)_{j \in \mathbb{N}}$ such that $A_{0}=A, A_{1}=V, A_{j+1}+A_{j+1} \subseteq A_{j}$ 
and $G_{0} \subseteq A_{j}$ for each $j$. Put $q_{j}=\left[A_{j-1}: A_{j}\right]$ for $1 \leq j \in \mathbb{N}$. Let $m, n$ and $\delta$ be the numbers guaranteed for $\alpha, \varepsilon, k=1$ and the sequence $\left(q_{j}\right)$ by Theorem 2.4.2. By the Transfer Principle they have to work for the hyperfinite abelian group $G$ and the internal sets $A_{0}, \ldots, A_{n}$ as well. Since $g$ is almost homomorphic on $G_{\mathrm{f}}$, it is $\delta$-homomorphic on $m A_{0} \subseteq G_{\mathrm{f}}$. It follows that there is an internal character $\gamma \in \widehat{G}$ such that $\left|\arg g(x) \gamma(x)^{-1}\right| \leq \varepsilon$ for $x \in A_{0}=A$. Obviously, $|\arg \gamma(x)| \leq \alpha+\varepsilon \leq \beta$ for $x \in A_{1}=V$, hence $\gamma \in \operatorname{Bohr}_{\beta}(V)$.

Let us close the cycle by proving that Theorem 2.4.1 implies Gordon's Conjecture 1 (Theorem 2.2.4), ie the surjectivity of the canonical embedding $\widehat{G}^{b} \rightarrow \widehat{G^{b}}$. In view of Proposition 1.2.3, the proof of this implication is plain.

Proof of 2.4.1 $\Rightarrow$ 2.2.4 Let $\left(G, G_{0}, G_{\mathrm{f}}\right)$ be a condensing IMG group triplet with a hyperfinite abelian ambient group $G$ and $\mathbf{G}=G_{\mathrm{f}} / G_{0}$ denote its observable trace. Assume that $\gamma: \mathbf{G} \rightarrow \mathbb{T}$ is a continuous character of $\mathbf{G}$. By Proposition 1.2.3, there is an internal mapping $g: D \rightarrow{ }^{*} \mathbb{T}$ such that $G_{\mathrm{f}} \subseteq D \subseteq G, g$ is $S$-continuous on $G_{\mathrm{f}}$, and

$$
\gamma\left(x^{b}\right)={ }^{\circ} g(x)
$$

for all $x \in G_{\mathrm{f}}$. Then

$$
g(x+y) \approx \gamma\left((x+y)^{b}\right)=\gamma\left(x^{b}+y^{b}\right)=\gamma\left(x^{b}\right) \gamma\left(y^{b}\right) \approx g(x) g(y)
$$

for all $x, y \in G_{\mathrm{f}}$, ie, $g$ is almost homomorphic on $G_{\mathrm{f}}$. By 2.4.1, there is a $\gamma \in G_{0}^{\downarrow}$ such that

$$
\gamma(x) \approx g(x) \approx \gamma\left(x^{b}\right)
$$

for each $x \in G_{\mathrm{f}}$, ie, $\gamma^{b}=\gamma$.

A brief inspection of the above proofs is instructive. In order to derive Theorem 2.4.2 from Theorem 2.2.4 it suffices to assume that Gordon's Conjecture 1 is true just for condensing IMG group triplets $\left(G, G_{0}, G_{\mathrm{f}}\right)$ with a hyperfinite $G$ (in some $\aleph_{1}$-saturated nonstandard universe), such that $G_{0}$ is an intersection and $G_{\mathrm{f}}$ is a union of countably many internal sets. This was indeed Gordon's original formulation of his conjecture in [17]. Conversely, in order to prove Theorem 2.4.1 (from which Theorem 2.2.4 easily follows by the virtue of Proposition 1.2.3) as a consequence of Theorem 2.4.2, it is enough to suppose that the latter is true only for a single fixed $\alpha \in(0,2 \pi / 3)$ and $k=1$.

Proposition 2.3.3, forming the essential part of the Triplet Duality Theorem 2.2.5, can be restated in standard terms as follows: 
Theorem 2.4.3 Let $\alpha, \beta \in(0,2 \pi / 3)$ and $\left(q_{j}\right)_{j=1}^{\infty}$ be any sequence of reals $q_{j} \geq 1$. Then there exists $n \in \mathbb{N}$, depending just on $\alpha, \beta$ and the sequence $\left(q_{j}\right)$, such that the following holds:

Let $G$ be a finite abelian group and $0 \in A_{n} \subseteq \ldots \subseteq A_{1} \subseteq A_{0} \subseteq G$ be symmetric sets such that

$$
A_{j}+A_{j} \subseteq A_{j-1} \quad \text { and } \quad\left[A_{j-1}: A_{j}\right] \leq q_{j}
$$

for $1 \leq j \leq n$. Then $\operatorname{Bohr}_{\beta}\left(\operatorname{Bohr}_{\alpha}\left(A_{n}\right)\right) \subseteq A_{0}$.

Theorem 2.4.3 can be derived from Proposition 2.3.3 using the ultraproduct construction, similarly as (and more easily than) the implication $2.2 .4 \Rightarrow 2.4 .2$. To this end it would be enough to assume that 2.3.3 is true just in case when $G_{0}$ is an intersection of a countable family of internal subsets of $G$, again. On the other hand, in order to derive 2.3.3 from 2.4.3, it would suffice to suppose that the latter is true for one fixed couple $\alpha, \beta \in(0,2 \pi / 3)$, only.

Remark Theorem 2.4.1 still holds for condensing IMG group triplets $\left(G, G_{0}, G_{\mathrm{f}}\right)$ with an arbitrary internal abelian ambient group $G$, and not just a hyperfinite one. Moreover, most of the results of Sections 2.2 and 2.3 admit analogous generalizations. Similarly, both Theorems 2.4.2 and 2.4.3 (and maybe even the strengthening of 2.4.2 mentioned in the remark following the proof of $2.4 .1 \Rightarrow 2.4 .2$ ) remain true when replacing "Let $G$ be a finite abelian group and $0 \in A_{n} \subseteq \ldots \subseteq A_{1} \subseteq A_{0} \subseteq G$ be symmetric sets..." by "Let $G$ be a Hausdorff LCA group and $A_{n} \subseteq \ldots \subseteq A_{1} \subseteq A_{0}$ be compact symmetric neighborhoods of 0 in G..." (without changing the rest) in their formulation.

In order to prove all that, it would be necessary (and sufficient) to deal with condensing IMG triplets of the form $\left(G, G_{0}, G_{\mathrm{f}}\right)$, where $G$ is an arbitrary internal Hausdorff LCA group, ie, a * (Hausdorff LCA) group, and define their dual triplets $\left(\widehat{G}, G_{\mathrm{f}}^{\downarrow}, G_{0}^{\downarrow}\right)$, with $\widehat{G}$ denoting the internal dual group of $G$, as well as to generalize the results of Section 2.1 from finite abelian groups to arbitrary Hausdorff LCA groups. That, however, though possible and - as we shall see in the next section - unavoidable to some extent, would be at odds with the leading intentions of the present paper, namely to study the LCA groups and the Fourier transform on functional spaces over them by means of their approximations by (hyper)finite abelian groups and the discrete Fourier transform on them.

That way generalized Theorem 2.4.3 can be schematically written as follows:

$$
(\forall \alpha, \beta)\left(\forall q_{1}, \ldots, q_{j}, \ldots\right)(\exists n)(\forall G)\left(\forall A_{0}, A_{1} \ldots, A_{n}\right)[(\forall j \leq n)(\ldots) \Rightarrow \ldots]
$$


However, from the Pontryagin-van Kampen Duality Theorem it follows only:

$$
(\forall \alpha, \beta)\left(\forall q_{1}, \ldots, q_{j}, \ldots\right)(\forall G)\left(\forall A_{0}, A_{1} \ldots, A_{j}, \ldots\right)[(\forall j)(\ldots) \Rightarrow(\exists n)(\ldots)]
$$

In other words, such a generalization of 2.4.3 adds a considerable uniformness to the above consequence of the Pontryagin-van Kampen Duality Theorem.

Analogous remarks could be made on behalf of the indicated generalizations of Theorems 2.4.1 and 2.4.2 and their relation to some standardly formulated stability results for characters of LCA groups with respect to the compact-open topology from Mačaj and Zlatoš [33] and Zlatoš [48]. However, the big amount of technicalities needed for their formulation and comparison would take us too far from the main lines and goals of the present paper.

\subsection{Simultaneous approximation of an LCA group and its dual}

Now, we are going to apply the results of the previous sections to triplets arising from HFI approximations of LCA groups. One can expect that an HFI approximation $\eta: G \rightarrow \mathbf{G}$ of an LCA group $\mathbf{G}$ gives rise to an HFI approximation $\phi: \widehat{G} \rightarrow \widehat{\mathbf{G}}$ of the dual group $\widehat{\mathbf{G}}$. This is true in some sense, however, in general, the connection between these two approximations is not as straightforward as one wished. Some of the material of this section is partly covered in the Introduction and §2.4 of Gordon's book [18].

As we will be dealing with plenty of more or less canonical isomorphisms of topological groups, first we have to make clear which of them we intend to exploit for identification of the isomorphic objects, and which of them we still view as isomorphisms of different objects.

Let $\mathbf{G}$ be a Hausdorff LCA group, viewed as a subgroup of its nonstandard extension ${ }^{*} \mathbf{G}$. Let us denote:

$$
\mathbb{I}^{*} \mathbf{G}=\operatorname{Mon}(0) \quad \text { and } \quad \mathbb{F}^{*} \mathbf{G}=\mathrm{Ns}\left({ }^{*} \mathbf{G}\right)
$$

Then $\left({ }^{*} \mathbf{G}, I^{*} \mathbf{G}, \mathbb{F}^{*} \mathbf{G}\right)$ is a condensing IMG group triplet with an internal abelian ambient group ${ }^{*} \mathbf{G}$. We identify the canonically isomorphic LCA groups $\mathbf{G}$ and the observable trace $\mathbb{F}^{*} \mathbf{G} / \mathbb{I}^{*} \mathbf{G}$ (nonstandard hull) of this triplet, as well as the standard part map $\boldsymbol{x} \mapsto{ }^{\circ} \boldsymbol{x}: \mathbb{F}^{*} \mathbf{G} \rightarrow \mathbf{G}$ and the observable trace map $\boldsymbol{x} \mapsto \boldsymbol{x}^{\mathrm{b}}: \mathbb{F}^{*} \mathbf{G} \rightarrow \mathbb{F}^{*} \mathbf{G} / \mathbb{I}^{*} \mathbf{G}$.

Similar, but less straightforward, identification applies also to the dual group $\widehat{\mathbf{G}}$. More specifically, due to the Transfer Principle, the nonstandard extension $* \widehat{\mathbf{G}}$ of the dual group $\widehat{\mathbf{G}}$ coincides with the internal dual group $\widehat{*} \widehat{\mathbf{G}}$ of ${ }^{*} \mathbf{G}$, consisting of all internal ${ }^{*}$ continuous characters $\gamma:{ }^{*} \mathbf{G} \rightarrow{ }^{*} \mathbb{T}$. However, the elementary embedding $\widehat{\mathbf{G}} \rightarrow{ }^{*} \widehat{\mathbf{G}}$ 
sends a character $\gamma: \mathbf{G} \rightarrow \mathbb{T}$ not literally to itself but to its nonstandard extension ${ }^{*} \boldsymbol{\gamma}:{ }^{*} \mathbf{G} \rightarrow{ }^{*} \mathbb{T}$. Putting

$$
\mathbb{I}^{*} \widehat{\mathbf{G}}=\operatorname{Mon}(1 * \mathbf{G}) \quad \text { and } \quad \mathbb{F}^{*} \widehat{\mathbf{G}}=\mathrm{Ns}\left({ }^{*} \widehat{\mathbf{G}}\right)
$$

one can easily realize that

$$
\begin{aligned}
& \mathbb{I}^{*} \widehat{\mathbf{G}}=\mathbb{F}^{*} \mathbf{G}^{\downarrow}=\left\{\boldsymbol{\gamma} \in{ }^{*} \widehat{\mathbf{G}}:\left(\forall \boldsymbol{x} \in \mathbb{F}^{*} \mathbf{G}\right)(\gamma(\boldsymbol{x}) \approx 1)\right\} \\
& \mathbb{F}^{*} \widehat{\mathbf{G}}=\mathbb{I}^{*} \mathbf{G}^{\downarrow}=\left\{\boldsymbol{\gamma} \in{ }^{*} \widehat{\mathbf{G}}:\left(\forall \boldsymbol{x} \in \mathbb{I}^{*} \mathbf{G}\right)(\gamma(\boldsymbol{x}) \approx 1)\right\}
\end{aligned}
$$

so that the condensing IMG group triplet $\left({ }^{*} \widehat{\mathbf{G}}, \mathbb{I} * \widehat{\mathbf{G}}, \mathbb{F} * \widehat{\mathbf{G}}\right)$ coincides with the dual triplet $\left(\widehat{{ }^{*} \mathbf{G}}, \mathbb{F}^{*} \mathbf{G}^{\downarrow}, \mathbb{I}^{*} \mathbf{G}^{\downarrow}\right)$ of $\left({ }^{*} \mathbf{G}, \mathbb{I}^{*} \mathbf{G}, \mathbb{F}^{*} \mathbf{G}\right)$. We identify the isomorphic LCA groups $\widehat{\mathbf{G}}$ and $\mathbb{F}^{*} \widehat{\mathbf{G}} / \mathbb{I} * \widehat{\mathbf{G}}$, as well as the standard part map $\gamma \mapsto{ }^{\circ} \gamma: \mathbb{F}^{*} \widehat{\mathbf{G}} \rightarrow \widehat{\mathbf{G}}$ and the observable trace map $\gamma \mapsto \gamma^{b}: \mathbb{F}^{*} \widehat{\mathbf{G}} \rightarrow \mathbb{F}^{*} \widehat{\mathbf{G}} / \mathbb{I} * \widehat{\mathbf{G}}$, again.

Now assume that $\eta: G \rightarrow{ }^{*} \mathbf{G}$ is an HFI approximation of $\mathbf{G}$ by a hyperfinite abelian group $G$ and $\left(G, G_{0}, G_{\mathrm{f}}\right)$ is the condensing IMG group triplet arising from this approximation, ie:

$$
G_{0}=\eta^{-1}\left[\mathbb{I}^{*} \mathbf{G}\right] \quad \text { and } \quad G_{\mathrm{f}}=\eta^{-1}\left[\mathbb{F}^{*} \mathbf{G}\right]
$$

Then $\eta:\left(G, G_{0}, G_{\mathrm{f}}\right) \rightarrow\left({ }^{*} \mathbf{G}, \mathbb{I}^{*} \mathbf{G}, \mathbb{F}^{*} \mathbf{G}\right)$ is a triplet isomorphism, and its observable trace $\boldsymbol{\eta}=\eta^{\mathrm{b}}: G_{\mathrm{f}} / G_{0} \rightarrow \mathbb{F}^{*} \mathbf{G} / \mathbb{I}^{*} \mathbf{G}$ becomes an isomorphism between the two representations of the original LCA group $\mathbf{G}$ as the observable trace $\mathbf{G} \cong G_{\mathrm{f}} / G_{0}$ and the nonstandard hull $\mathbf{G} \cong \mathbb{F}^{*} \mathbf{G} / \mathbb{I}^{*} \mathbf{G}$.

Let us form also the dual triplet $\left(\widehat{G}, G_{\mathrm{f}}^{\downarrow}, G_{0}^{\downarrow}\right)$. Making use of the canonical isomorphism $G_{0}^{\nu} / G_{\mathrm{f}}^{\downarrow} \rightarrow \widehat{G_{\mathrm{f}} / G_{0}}$ from Theorem 2.2.4 we identify the dual group $\widehat{G_{\mathrm{f}} / G_{0}}$ of the observable trace $G^{b}=G_{\mathrm{f}} / G_{0}$ and the observable trace $\widehat{G}^{b}=G_{0}^{\downarrow} / G_{\mathrm{f}}^{\downarrow}$ of the dual triplet $\left(\widehat{G}, G_{\mathrm{f}}^{\downarrow}, G_{0}^{\downarrow}\right)$.

Keeping in mind the just introduced identifications and applying the duality functor to the isomorphism $\eta^{-1}: \mathbf{G} \rightarrow G_{\mathrm{f}} / G_{0}$ of LCA groups, we obtain the isomorphism $\phi: G_{0}^{\downarrow} / G_{\mathrm{f}}^{\downarrow} \rightarrow \widehat{\mathbf{G}}$ between their duals, given by

$$
\phi(\gamma)=\gamma \circ \eta^{-1}
$$

for $\gamma \in \widehat{G_{\mathrm{f}} / G_{0}}=G_{0}^{\downarrow} / G_{\mathrm{f}}^{\downarrow}$.

By Corollary 1.2.4, any isomorphism $\phi: G_{0}^{\downarrow} / G_{\mathrm{f}}^{\downarrow} \rightarrow \mathbb{F}^{*} \widehat{\mathbf{G}} / \mathbb{I}^{*} \widehat{\mathbf{G}}$ between the two representations of the dual group $\widehat{\mathbf{G}} \cong G_{0}^{\downarrow} / G_{\mathrm{f}}^{\downarrow}$ and $\widehat{\mathbf{G}} \cong \mathbb{F} * \widehat{\mathbf{G}} / \mathbb{I} * \widehat{\mathbf{G}}$ is the observable trace of some internal mapping $\phi: \widehat{G} \rightarrow{ }^{*} \widehat{\mathbf{G}}$. This is to say that $\phi:\left(\widehat{G}, G_{\mathrm{f}}^{\downarrow}, G_{0}^{\downarrow}\right) \rightarrow$ $(* \widehat{\mathbf{G}}, \mathbb{I} * \widehat{\mathbf{G}}, \mathbb{F} * \widehat{\mathbf{G}})$ is a triplet isomorphism and

$$
\phi\left(\gamma^{b}\right)=\gamma^{b} \circ \eta^{-1}=\phi(\gamma)^{b}
$$


for $\gamma \in G_{0}^{\downarrow}$. Then $\phi$ necessarily is almost homomorphic on $G_{0}^{\downarrow}$, and we have

$$
G_{\mathrm{f}}^{\downarrow}=\phi^{-1}\left[\mathbb{I}^{*} \widehat{\mathbf{G}}\right] \quad \text { and } \quad G_{0}^{\downarrow}=\phi^{-1}\left[\mathbb{F}^{*} \widehat{\mathbf{G}}\right]
$$

so that $\phi: \widehat{G} \rightarrow{ }^{*} \widehat{\mathbf{G}}$ is an HFI approximation of $\widehat{\mathbf{G}}$ and $\left(\widehat{G}, G_{\mathrm{f}}^{\downarrow}, G_{0}^{\downarrow}\right)$ is the IMG group triplet arising from this approximation. In the particular case of $\phi$ given by the assignment $\boldsymbol{\gamma} \mapsto \boldsymbol{\gamma} \circ \boldsymbol{\eta}^{-1}$, as above, we finally have

$$
(\phi \gamma)(\eta x) \approx\left(\phi \gamma^{b}\right)\left(\boldsymbol{\eta} x^{b}\right)=\left(\gamma^{b} \circ \boldsymbol{\eta}^{-1}\right)\left(\boldsymbol{\eta} x^{b}\right)=\gamma^{b}\left(x^{b}\right) \approx \gamma(x)
$$

for any $x \in G_{\mathrm{f}}, \gamma \in G_{0}^{\downarrow}$.

Remark Though this is not an essential point, in view of Corollary 1.3 .11 we can assume, for convenience' sake, that the approximation $\eta$ is injective and preserves 0 and inverses. Then there is some internal, necessarily surjective mapping $\eta^{\prime}:{ }^{*} \mathbf{G} \rightarrow G$ such that $\eta^{\prime} \circ \eta=\operatorname{Id}_{G}, \boldsymbol{x} \approx\left(\eta \circ \eta^{\prime}\right)(\boldsymbol{x})$, for $\boldsymbol{x} \in \mathbb{F}^{*} \mathbf{G}$, and $\eta^{\prime}$ preserves 0 and inverses, as well. Now, it is natural to define the mapping $\phi$ by the assignment $\gamma \mapsto \gamma \circ \eta^{\prime}$, for $\gamma \in \widehat{G}$. Such a $\phi$ would be injective and strictly preserving the pointwise multiplication of functions (hence the trivial character $1_{G}$ and pointwise inverses, too). Unfortunately, this natural attempt does not work. The reason is that $\eta, \eta^{\prime}$, in spite of being almost homomorphic on $G_{\mathrm{f}}, \mathbb{F}^{*} \mathbf{G}$, respectively, are not genuine homomorphisms, in general. Hence the mapping $\gamma \circ \eta^{\prime}$ (though preserving 0 and inverses) would be just almost homomorphic on $\mathbb{F}^{*} \mathbf{G}$, again, and one cannot assure that $\gamma \circ \eta^{\prime} \in{ }^{*} \widehat{\mathbf{G}}$, for $\gamma \in \widehat{G}$. Thus there seems to be no canonical way how to determine the HFI approximation $\phi: \widehat{G} \rightarrow{ }^{*} \widehat{\mathbf{G}}$ of $\widehat{\mathbf{G}}$ right away from the HFI approximation $\eta: G \rightarrow{ }^{*} \mathbf{G}$ of $\mathbf{G}$.

On the other hand, for each $\gamma \in \widehat{\mathbf{G}}$, the composition ${ }^{*} \gamma \circ \eta: G \rightarrow{ }^{*} \mathbb{T}$ is almost homomorphic on $G_{\mathrm{f}}$ (in fact, it is an $S$-continuous lifting of $\gamma$ ). By Theorem 2.2.4, there is a genuine homomorphism $\gamma \in G_{0}^{\downarrow} \subseteq \widehat{G}$ such that $\gamma=\left({ }^{*} \gamma \circ \eta\right)^{b}=\gamma^{b}$; more precisely,

$$
\left({ }^{*} \gamma \circ \eta\right)(x) \approx \gamma(x)
$$

for each $x \in G_{\mathrm{f}}$. Though ${ }^{*} \gamma \circ \eta \notin \widehat{G}$, in general, we shall see in our following paper [50] that it can be used directly instead of the genuine homomorphism $\gamma \in G_{0}^{\downarrow}$ in approximation of the Fourier transform on $\mathbf{G}$ by means of the inner product on the hyperfinite dimensional unitary space ${ }^{*} \mathbb{C}^{G}$.

Now assume that $G$ and $H$ are hyperfinite abelian groups, $\eta: G \rightarrow{ }^{*} \mathbf{G}$ is an HFI approximation of the Hausdorff LCA group $\mathbf{G}$ and $\phi: H \rightarrow{ }^{*} \widehat{\mathbf{G}}$ is an HFI approximation of its dual group $\widehat{\mathbf{G}}$. Let $\left(G, G_{0}, G_{\mathrm{f}}\right)$ and $\left(H, H_{0}, H_{\mathrm{f}}\right)$ be the condensing IMG triplets arising from these approximations, ie:

$$
G_{0}=\eta^{-1}\left[\mathbb{I}^{*} \mathbf{G}\right] \quad G_{\mathrm{f}}=\eta^{-1}\left[\mathbb{F}^{*} \mathbf{G}\right]
$$




$$
H_{0}=\phi^{-1}[\mathbb{I} * \widehat{\mathbf{G}}] \quad H_{\mathrm{f}}=\phi^{-1}[\mathbb{F} * \widehat{\mathbf{G}}]
$$

Following Gordon [18, page 148] we say that the approximations $\eta$ and $\phi$ are dual to each other or that they form an adjoint pair if $H=\widehat{G}, H_{0}=G_{\mathrm{f}}^{\downarrow}, H_{\mathrm{f}}=G_{0}^{\downarrow}$, ie if the triplet $\left(H, H_{0}, H_{\mathrm{f}}\right)$ coincides with the dual triplet $\left(\widehat{G}, G_{\mathrm{f}}^{\downarrow}, G_{0}^{\downarrow}\right)$ of $\left(G, G_{0}, G_{\mathrm{f}}\right)$, and

$$
(\phi \gamma)(\eta x) \approx \gamma(x)
$$

holds for all $x \in G_{\mathrm{f}}, \gamma \in H_{\mathrm{f}}$. Inspecting our accounts and making use of the notions just introduced, we find that, in any sufficiently saturated nonstandard universe, we have proved the following results.

Theorem 2.5.1 Let $\left(G, G_{0}, G_{\mathrm{f}}\right)$ be an IMG group triplet with a hyperfinite abelian ambient group $G$, arising from an HFI approximation $\eta: G \rightarrow{ }^{*} \mathbf{G}$ of the Hausdorff LCA group $\mathbf{G}$, and the isomorphism $\eta: G_{\mathrm{f}} / G_{0} \rightarrow \mathbf{G}$ of LCA groups be the observable trace of $\eta$. Let further $\phi: \widehat{G} \rightarrow{ }^{*} \widehat{\mathbf{G}}$ be an HFI approximation of the dual LCA group $\widehat{\mathbf{G}}$ such that its observable trace $\phi=\phi^{b}: G_{0}^{\downarrow} / G_{\mathrm{f}}^{\downarrow} \rightarrow \widehat{\mathbf{G}}$ is the dual isomorphism corresponding to $\eta^{-1}: \mathbf{G} \rightarrow G_{\mathrm{f}} / G_{0}$. Then the HFI approximations $\eta$ and $\phi$ are dual to each other.

Corollary 2.5.2 (The Adjoint Hyperfinite LCA Group Approximation Theorem) To every HFI approximation $\eta: G \rightarrow{ }^{*} \mathbf{G}$ of a Hausdorff $L C A$ group $\mathbf{G}$ by a hyperfinite abelian group $G$ there exists a dual HFI approximation $\phi: \widehat{G} \rightarrow{ }^{*} \widehat{\mathbf{G}}$ of the dual LCA group $\widehat{\mathbf{G}}$ by the dual hyperfinite abelian group $\widehat{G}$.

On the other hand, even if $\eta$ is injective, our accounts, so far, are not sufficient to establish the analogous property for $\phi$.

It is worthwhile to realize that, in a sufficiently saturated nonstandard universe, the conditions defining the notion of an adjoint pair of HFI approximations are redundant to some extent. Here is a more detailed account of their relation.

Lemma 2.5.3 Let $G$ be a hyperfinite abelian group, $\eta: G \rightarrow{ }^{*} \mathbf{G}, \phi: \widehat{G} \rightarrow{ }^{*} \widehat{\mathbf{G}}$ be HFI approximations of the Hausdorff LCA groups $\mathbf{G}, \widehat{\mathbf{G}}$, respectively, and $\left(G, G_{0}, G_{\mathrm{f}}\right)$, $\left(\widehat{G}, H_{0}, H_{\mathrm{f}}\right)$ be the condensing IMG triplets arising from these approximations, such that

$$
(\phi \gamma)(\eta x) \approx \gamma(x)
$$

for all $x \in G_{\mathrm{f}}, \gamma \in H_{\mathrm{f}}$. Then, as consequence, the following two conditions hold:

(a) The inclusions $H_{\mathrm{f}} \subseteq G_{0}^{\downarrow}, G_{\mathrm{f}} \subseteq H_{0}^{\downarrow}, G_{0} \subseteq H_{\mathrm{f}}^{\downarrow}$, and $H_{0} \subseteq G_{\mathrm{f}}^{\downarrow}$ are satisfied. 
(b) Any two of the reversed inclusions $G_{0}^{\downarrow} \subseteq H_{\mathrm{f}}, H_{0}^{\downarrow} \subseteq G_{\mathrm{f}}, H_{\mathrm{f}}^{\downarrow} \subseteq G_{0}$, and $G_{\mathrm{f}}^{\downarrow} \subseteq H_{0}$ are equivalent.

Proof (a) In order to prove the first inclusion, take any $\gamma \in H_{\mathrm{f}}$. As $H_{\mathrm{f}}=\phi^{-1}\left[\mathbb{F}^{*} \widehat{\mathbf{G}}\right]$ and $\mathbb{F}^{*} \widehat{\mathbf{G}}=\mathbb{I}^{*} \mathbf{G}^{\downarrow}$, we have $(\phi \gamma)(\boldsymbol{x}) \approx 1$ for each $\boldsymbol{x} \in \mathbb{I}^{*} \mathbf{G}=\operatorname{Mon}(0)$. Since $G_{0}=\eta^{-1}\left[\mathbb{I}^{*} \mathbf{G}\right]$, this implies $(\phi \gamma)(\eta x) \approx 1$ for each $x \in G_{0}$. From $G_{0} \subseteq G_{\mathrm{f}}$ we obtain

$$
\gamma(x) \approx(\phi \gamma)(\eta x) \approx 1
$$

hence $\gamma \in G_{0}^{\downarrow}$. From $H_{\mathrm{f}} \subseteq G_{0}^{\downarrow}$ we readily get $G_{0} \subseteq G_{0}^{\downarrow} \sim \subseteq H_{\mathrm{f}}^{\downarrow}$. The third and the forth inclusion now follow by the symmetry of the situation.

(b) For brevity's sake let us number the inclusions consecutively from (i) to (iv). First we prove (i) $\Rightarrow$ (iv). Assume that $G_{0}^{\downarrow} \subseteq H_{\mathrm{f}}$ and take any $\gamma \in G_{\mathrm{f}}^{\downarrow} \subseteq G_{0}^{\downarrow} \subseteq H_{\mathrm{f}}$. Then $\phi(\gamma) \in \mathbb{F}^{*} \widehat{\mathbf{G}}=\mathbb{I}^{*} \mathbf{G}^{\nu}$, ie, $\phi(\gamma)$ is $S$-continuous. As $H_{0}=\phi^{-1}\left[\mathbb{I}^{*} \widehat{\mathbf{G}}\right]$ and $\mathbb{I}^{*} \widehat{\mathbf{G}}=\mathbb{F}^{*} \mathbf{G}^{\downarrow}$, in order to establish that $\gamma \in H_{0}$, we are to show that $(\phi \gamma)(\boldsymbol{x}) \approx 1$ for each $\left.\boldsymbol{x} \in \mathbb{F}^{*} \mathbf{G}=\mathrm{Ns}^{*} \mathbf{G}\right)$. Since there is $x \in G_{\mathrm{f}}$ such that $\boldsymbol{x} \approx \eta(x)$, we have indeed:

$$
(\phi \gamma)(x) \approx(\phi \gamma)(\eta x) \approx \gamma(x) \approx 1
$$

(ii) $\Rightarrow$ (iii) can be proved analogously by a symmetry argument.

Next we show (iv) $\Rightarrow$ (ii). From $G_{\mathrm{f}}^{\sim} \subseteq H_{0}$ we get $H_{0}^{\sim} \subseteq G_{\mathrm{f}}^{\sim} \sim=G_{\mathrm{f}}$ by the Triplet Duality Theorem 2.2.5 (see also Lemma 2.2.7). By symmetry we have (iii) $\Rightarrow$ (i) as well, closing the circle of implications.

In other words, under the assumptions of the lemma, the HFI approximations $\eta$ and $\phi$ form an adjoint pair if and only if any (hence all) of the inclusions from (b) is true. We are inclining to consider the first inclusion $G_{0}^{\downarrow} \subseteq H_{\mathrm{f}}$ from (b) as intuitively the most appealing and fundamental one. In "unzipped form" it states that, for all $\gamma \in \widehat{G}$,

$$
\left(\forall x \in G_{0}\right)(\gamma(x) \approx 1) \Rightarrow\left(\forall \boldsymbol{x} \in \mathbb{I}^{*} \mathbf{G}\right)((\phi \gamma)(\boldsymbol{x}) \approx 1)
$$

and, as $\gamma(x) \approx(\phi \gamma)(\eta x)$, it is equivalent to:

$$
\left(\forall \boldsymbol{x} \in \eta\left[G_{0}\right]\right)((\phi \gamma)(\boldsymbol{x}) \approx 1) \Rightarrow\left(\forall \boldsymbol{x} \in \mathbb{I}^{*} \mathbf{G}\right)((\phi \gamma)(\boldsymbol{x}) \approx 1)
$$

The remaining inclusions in (b) can be "unzipped" in a similar way.

The standard meaning of the above accounts and results can be formulated in terms of adjoint pairs of finite approximations. The equivalence of both formulations could be established by referring to Nelson's translation algorithm [37]. However, this would obscure some connections, so we offer a more detailed exposition. 
Let $\mathbf{G}$ be a Hausdorff LCA group and $0<\alpha<2 \pi / 3$. Then a $\mathbf{G}-\operatorname{raster}(\mathbf{K}, \mathbf{U})$ and a $\widehat{\mathbf{G}}$-raster $(\boldsymbol{\Gamma}, \boldsymbol{\Omega})$ are called $\alpha$-adjoint if

$$
\mathbf{U} \subseteq \operatorname{Bohr}_{\alpha}(\boldsymbol{\Gamma}) \quad \text { and } \quad \boldsymbol{\Omega} \subseteq \operatorname{Bohr}_{\alpha}(\mathbf{K})
$$

which means that $|\arg \gamma(\boldsymbol{x})| \leq \alpha$ for any $\boldsymbol{x} \in \mathbf{U}, \gamma \in \Gamma$, as well as for any $\boldsymbol{x} \in \mathbf{K}$, $\gamma \in \boldsymbol{\Omega}$. In particular, any compact neighborhoods $\mathbf{K}, \boldsymbol{\Gamma}$ of the neutral elements in $\mathbf{G}$, $\widehat{\mathbf{G}}$, respectively, give rise to an $\alpha$-adjoint pair of rasters $(\mathbf{K}, \mathbf{U}),(\boldsymbol{\Gamma}, \boldsymbol{\Omega})$ when putting

$$
\mathbf{U}=\operatorname{Bohr}_{\alpha}(\boldsymbol{\Gamma}) \cap \mathbf{K} \quad \text { and } \quad \boldsymbol{\Omega}=\operatorname{Bohr}_{\alpha}(\mathbf{K}) \cap \boldsymbol{\Gamma}
$$

If $G$ is a finite abelian group then a $\mathbf{G}$-raster $(\mathbf{K}, \mathbf{U})$ and a $\widehat{\mathbf{G}}$-raster $(\boldsymbol{\Gamma}, \boldsymbol{\Omega})$ together with a pair of mappings $\eta: G \rightarrow \mathbf{G}, \phi: \widehat{G} \rightarrow \widehat{\mathbf{G}}$ are said to form an $\alpha$-adjoint approximation scheme for the pair of groups $\mathbf{G}, \widehat{\mathbf{G}}$ if $\eta$ is a $(\mathbf{K}, \mathbf{U})$ approximation of $\mathbf{G}, \phi$ is a $(\boldsymbol{\Gamma}, \boldsymbol{\Omega})$ approximation of $\widehat{\mathbf{G}}$ and the two rasters are $\alpha$-adjoint.

A $(\mathbf{K}, \mathbf{U})$ approximation $\eta: G \rightarrow \mathbf{G}$ and a $(\boldsymbol{\Gamma}, \boldsymbol{\Omega})$ approximation $\phi: \widehat{G} \rightarrow \widehat{\mathbf{G}}$ are called pairing preserving on the set $\mathbf{K} \times \boldsymbol{\Gamma}$ if

$$
(\phi \gamma)(\eta x)=\gamma(x)
$$

for any $x \in \eta^{-1}[\mathbf{K}], \gamma \in \phi^{-1}[\boldsymbol{\Gamma}]$. An $\alpha$-adjoint approximation scheme is called pairing preserving if the approximations $\eta, \phi$ are pairing preserving on $\mathbf{K} \times \Gamma$.

Let further $\alpha>0, \varepsilon \geq 0$ satisfy $\alpha+\varepsilon<2 \pi / 3$. Then we say that the (K, U) approximation $\eta: G \rightarrow \mathbf{G}$ and the $(\boldsymbol{\Gamma}, \boldsymbol{\Omega})$ approximation $\phi: \widehat{G} \rightarrow \widehat{\mathbf{G}}$ are $\varepsilon$-pairing preserving on the set $\mathbf{K} \times \boldsymbol{\Gamma}$ if

$$
\left|\arg \frac{(\phi \gamma)(\eta x)}{\gamma(x)}\right| \leq \varepsilon
$$

for any $x \in \eta^{-1}[\mathbf{K}], \gamma \in \phi^{-1}[\boldsymbol{\Gamma}]$. An $\alpha$-adjoint approximation scheme is called $\varepsilon$-pairing preserving if the approximations $\eta, \phi$ are $\varepsilon$-pairing preserving on $\mathbf{K} \times \boldsymbol{\Gamma}$. Formally, pairing preserving approximations can be considered as a 0 -pairing preserving ones. For technical reasons we introduce also a slight strengthening of this notion. Such an $\alpha$-adjoint approximation scheme is called $\varepsilon$-pairing preserving with reserve if the approximations $\eta, \phi$ are $\varepsilon$-pairing preserving even on the set $(\mathbf{K}+\mathbf{U}) \times(\boldsymbol{\Gamma} \Omega)$.

The following inclusions form the standard analogue of part (a) of Lemma 2.5.3.

Lemma 2.5.4 Let $\mathbf{G}$ be a Hausdorff LCA group, $G$ be a finite abelian group and $\alpha>0, \varepsilon \geq 0$ be such that $\alpha+\varepsilon<2 \pi / 3$. If $(\mathbf{K}, \mathbf{U}),(\boldsymbol{\Gamma}, \boldsymbol{\Omega})$ are rasters and $\eta: G \rightarrow \mathbf{G}$, $\phi: \widehat{G} \rightarrow \widehat{\mathbf{G}}$ are mappings forming an $\alpha$-adjoint $\varepsilon$-pairing preserving approximation scheme of the pair $\mathbf{G}, \widehat{\mathbf{G}}$ then

$$
\begin{aligned}
\phi^{-1}[\boldsymbol{\Gamma}] \subseteq \operatorname{Bohr}_{\alpha+\varepsilon}\left(\eta^{-1}[\mathbf{U}]\right) & \eta^{-1}[\mathbf{K}] \subseteq \operatorname{Bohr}_{\alpha+\varepsilon}\left(\phi^{-1}[\Omega]\right) \\
\eta^{-1}[\mathbf{U}] \subseteq \operatorname{Bohr}_{\alpha+\varepsilon}\left(\phi^{-1}[\boldsymbol{\Gamma}]\right) & \phi^{-1}[\Omega] \subseteq \operatorname{Bohr}_{\alpha+\varepsilon}\left(\eta^{-1}[\mathbf{K}]\right)
\end{aligned}
$$


Proof We will prove just the upper left inclusion; the lower left inclusion is equivalent to it, while the inclusions in the right column follow from those in the left one by a symmetry argument. We have to show that $|\arg \gamma(x)| \leq \alpha+\varepsilon$ for any $x \in \eta^{-1}[\mathbf{U}]$, $\gamma \in \phi^{-1}[\Gamma]$. In that case we have

$$
|\arg \gamma(x)| \leq|\arg (\phi \gamma)(\eta x)|+\left|\arg \frac{\gamma(x)}{(\phi \gamma)(\eta x)}\right| \leq \alpha+\varepsilon
$$

since $\eta(x) \in \mathbf{U}, \phi(\gamma) \in \boldsymbol{\Gamma}$ and $\mathbf{U} \subseteq \operatorname{Bohr}_{\alpha}(\boldsymbol{\Gamma})$.

In order to take care of (the first two of) the four equivalent conditions formulated in part (b) of Lemma 2.5.3, we need to introduce one more notion. Assume that $(\mathbf{Q}, \mathbf{V})$ is a $\mathbf{G}$-raster and $(\boldsymbol{\Delta}, \boldsymbol{\Upsilon})$ is a $\widehat{\mathbf{G}}$-raster, given in advance. Let $\mathbf{G}$ and $\alpha$ be as above. We say that an $\alpha$-adjoint approximation scheme of the pair of groups $\mathbf{G}, \widehat{\mathbf{G}}$ given by some rasters $(\mathbf{K}, \mathbf{U}),(\boldsymbol{\Gamma}, \boldsymbol{\Omega})$ and mappings $\eta: G \rightarrow \mathbf{G}, \phi: \widehat{G} \rightarrow \widehat{\mathbf{G}}$ is compatible with the rasters $(\mathbf{Q}, \mathbf{V}),(\boldsymbol{\Delta}, \boldsymbol{\Upsilon})$ if $(\mathbf{Q}, \mathbf{V}) \leq(\mathbf{K}, \mathbf{U}),(\boldsymbol{\Delta}, \boldsymbol{\Upsilon}) \leq(\boldsymbol{\Gamma}, \boldsymbol{\Omega})$, and

$$
\operatorname{Bohr}_{\alpha}\left(\eta^{-1}[\mathbf{V}]\right) \subseteq \phi^{-1}[\Gamma] \quad \operatorname{Bohr}_{\alpha}\left(\phi^{-1}[\mathbf{\Upsilon}]\right) \subseteq \eta^{-1}[\mathbf{K}]
$$

Obviously, if some approximation scheme is $\alpha$-adjoint, $\varepsilon$-pairing preserving and compatible with a pair of rasters $(\mathbf{Q}, \mathbf{V}),(\boldsymbol{\Delta}, \mathbf{\Upsilon})$, then it is $\varepsilon^{\prime}$-pairing preserving for any $\varepsilon^{\prime} \geq \varepsilon$ and compatible with any pair of rasters $\left(\mathbf{Q}^{\prime}, \mathbf{V}^{\prime}\right) \leq(\mathbf{Q}, \mathbf{V}),\left(\boldsymbol{\Delta}^{\prime}, \boldsymbol{\Upsilon}^{\prime}\right) \leq(\boldsymbol{\Delta}, \boldsymbol{\Upsilon})$.

Theorem 2.5.5 (The Adjoint Hyperfinite Approximation Scheme Theorem) Let $\mathbf{G}$ be a Hausdorff LCA group. Then for every $\mathbf{G}-\operatorname{raster}(\mathbf{Q}, \mathbf{V})$, every $\widehat{\mathbf{G}}-\operatorname{raster}(\boldsymbol{\Delta}, \mathbf{\Upsilon})$ and all $\alpha, \varepsilon>0$ such that $\alpha+\varepsilon<2 \pi / 3$, there exists an $\alpha$-adjoint approximation scheme of the pair of groups $\mathbf{G}, \widehat{\mathbf{G}}$ given by some rasters $(\mathbf{K}, \mathbf{U}),(\boldsymbol{\Gamma}, \mathbf{\Omega})$, a finite abelian group $G$ and mappings $\eta: G \rightarrow \mathbf{G}, \phi: \widehat{G} \rightarrow \widehat{\mathbf{G}}$, which is $\varepsilon$-pairing preserving with reserve and compatible with the rasters $(\mathbf{Q}, \mathbf{V}),(\boldsymbol{\Delta}, \mathbf{\Upsilon})$. One can arrange, additionally, that at least one of the approximations $\eta, \phi$ is injective.

Proof According to Corollaries 1.3.11 and 2.5.2, (in a sufficiently saturated nonstandard universe) there is a hyperfinite abelian group $G$ and an adjoint pair of HFI approximations $\zeta: G \rightarrow{ }^{*} \mathbf{G}, \psi: \widehat{G} \rightarrow{ }^{*} \widehat{\mathbf{G}}$. Denote by $\left(G, G_{0}, G_{\mathrm{f}}\right),\left(\widehat{G}, H_{0}, H_{\mathrm{f}}\right)$ the corresponding IMG group triplets arising from $\zeta, \psi$, respectively. Let $(\mathbf{Q}, \mathbf{V}),(\boldsymbol{\Delta}, \mathbf{\Upsilon})$, and $\alpha, \varepsilon$ satisfy the assumptions of the theorem. Then, for any $\mathbf{G}-\operatorname{raster}(\mathbf{K}, \mathbf{U})$ and any $\widehat{\mathbf{G}}-\operatorname{raster}(\boldsymbol{\Gamma}, \boldsymbol{\Omega})$, which are $\alpha$-adjoint, $\left({ }^{*} \mathbf{K},{ }^{*} \mathbf{U}\right)\left({ }^{*} \boldsymbol{\Gamma},{ }^{*} \boldsymbol{\Omega}\right)$, together with $\zeta$ and $\psi$, form an $\alpha$-adjoint approximation scheme of the pair of groups ${ }^{*} \mathbf{G},{ }^{*} \widehat{\mathbf{G}}$ which is $\varepsilon$-pairing preserving with reserve. By saturation, there are an internal ${ }^{*} \mathbf{G}$-raster $\left(\mathbf{K}^{\prime}, \mathbf{U}^{\prime}\right)$ and an internal ${ }^{*} \widehat{\mathbf{G}}-$ raster $\left(\boldsymbol{\Gamma}^{\prime}, \boldsymbol{\Omega}^{\prime}\right)$ such that

$$
\mathbf{U}^{\prime} \subseteq \mathbb{I}^{*} \mathbf{G} \subseteq \mathbb{F}^{*} \mathbf{G} \subseteq \mathbf{K}^{\prime} \quad \text { and } \quad \mathbf{\Omega}^{\prime} \subseteq \mathbb{I}^{*} \widehat{\mathbf{G}} \subseteq \mathbb{F}^{*} \widehat{\mathbf{G}} \subseteq \Gamma^{\prime}
$$


still forming, together with $\zeta, \psi$, an $\alpha$-adjoint $\varepsilon$-pairing preserving with reserve approximation scheme for the pair of groups ${ }^{*} \mathbf{G},{ }^{*} \widehat{\mathbf{G}}$, Then $\left({ }^{*} \mathbf{Q},{ }^{*} \mathbf{V}\right) \leq\left(\mathbf{K}^{\prime}, \mathbf{U}^{\prime}\right)$ and $\left({ }^{*} \Delta,{ }^{*} \Upsilon\right) \leq\left(\Gamma^{\prime}, \Omega^{\prime}\right)$. At the same time,

$$
\begin{aligned}
& \operatorname{Bohr}_{\alpha}\left(\zeta^{-1}\left[{ }^{*} \mathbf{V}\right]\right) \subseteq G_{0}^{\downarrow}=H_{\mathrm{f}}=\psi^{-1}\left[\mathbb{F}^{*} \widehat{\mathbf{G}}\right] \subseteq \psi^{-1}\left[\boldsymbol{\Gamma}^{\prime}\right] \\
& \operatorname{Bohr}_{\alpha}\left(\psi^{-1}\left[{ }^{*} \Upsilon \mathbf{\Upsilon}\right] \subseteq H_{0}^{\downarrow}=G_{\mathrm{f}}=\zeta^{-1}\left[\mathbb{F}^{*} \mathbf{G}\right] \subseteq \zeta^{-1}\left[\mathbf{K}^{\prime}\right]\right.
\end{aligned}
$$

showing that the internal approximation scheme of the pair ${ }^{*} \mathbf{G},{ }^{*} \widehat{\mathbf{G}}$, given by $\left(\mathbf{K}^{\prime}, \mathbf{U}^{\prime}\right)$, $\left(\boldsymbol{\Gamma}^{\prime}, \boldsymbol{\Omega}^{\prime}\right)$ and $G, \zeta, \psi$, is compatible with the rasters $\left({ }^{*} \mathbf{Q},{ }^{*} \mathbf{V}\right),\left({ }^{*} \boldsymbol{\Delta},{ }^{*} \boldsymbol{\Upsilon}\right)$. According to the Transfer Principle, there is some $\alpha$-adjoint $\varepsilon$-pairing preserving with reserve approximation scheme of the pair $\mathbf{G}, \widehat{\mathbf{G}}$, given by some rasters $(\mathbf{K}, \mathbf{U}) \geq(\mathbf{Q}, \mathbf{V})$, $(\boldsymbol{\Gamma}, \boldsymbol{\Omega}) \geq(\boldsymbol{\Delta}, \boldsymbol{\Upsilon})$, a finite abelian group $G$ and mappings $\eta: G \rightarrow \mathbf{G}, \phi: \widehat{G} \rightarrow \widehat{\mathbf{G}}$, compatible with the original rasters $(\mathbf{Q}, \mathbf{V}),(\boldsymbol{\Delta}, \mathbf{\Upsilon})$.

If at least one of the starting HFI approximations $\zeta, \psi$ is injective then one can guarantee the same property for the corresponding finite approximation $\eta, \phi$, respectively, as well.

The Adjoint Hyperfinite Approximation Scheme Theorem 2.5.5 can be alternatively formulated in terms of convergent systems of adjoint approximation schemes (cf Proposition 1.3.7).

Let $\mathbf{G}$ be an LCA group, $0<\alpha<2 \pi / 3$ and $(I, \leq)$ be a directed poset. Assume that, for each $i \in I$, there are a $\mathbf{G}$-raster $\left(\mathbf{K}_{i}, \mathbf{U}_{i}\right)$, a $\widehat{\mathbf{G}}$-raster $\left(\boldsymbol{\Gamma}_{i}, \boldsymbol{\Omega}_{i}\right)$, a finite abelian group $G_{i}$ and a pair of mappings $\eta_{i}: G_{i} \rightarrow \mathbf{G}, \phi_{i}: \widehat{G}_{i} \rightarrow \widehat{\mathbf{G}}$ forming an $\alpha$-adjoint approximation scheme for the pair $\mathbf{G}, \widehat{\mathbf{G}}$. We say that this system is convergent if for every $\mathbf{G}-\operatorname{raster}(\mathbf{Q}, \mathbf{V})$, every $\widehat{\mathbf{G}}-\operatorname{raster}(\boldsymbol{\Delta}, \boldsymbol{\Upsilon})$ and each $\varepsilon \in(0,2 \pi / 3-\alpha)$, there is $i \in I$ such that the approximation scheme given by the rasters $\left(\mathbf{K}_{j}, \mathbf{U}_{j}\right),\left(\boldsymbol{\Gamma}_{j}, \boldsymbol{\Omega}_{j}\right)$, and the mappings $\eta_{j}: G_{j} \rightarrow \mathbf{G}, \phi_{j}: \widehat{G}_{j} \rightarrow \widehat{\mathbf{G}}$ is $\varepsilon$-pairing preserving and compatible with the rasters $(\mathbf{Q}, \mathbf{V}),(\boldsymbol{\Delta}, \mathbf{\Upsilon})$, for any $j \geq i$.

It is clear that in such a case the systems $\left(\left(\mathbf{K}_{i}, \mathbf{U}_{i}\right)\right)_{i \in I},\left(\left(\boldsymbol{\Gamma}_{i}, \Omega_{i}\right)\right)_{i \in I}$ are raster bases of the LCA groups $\mathbf{G}, \widehat{\mathbf{G}}$, respectively, while $\left(\eta_{i}\right)_{i \in I}$ is an approximating system of $\mathbf{G}$ and $\left(\phi_{i}\right)_{i \in I}$ is an approximating system of its dual group $\widehat{\mathbf{G}}$.

The following result slightly generalizes and gives more precision to Theorem 3 stated without proof in the introduction to Gordon [18].

Theorem 2.5.6 Let $\mathbf{G}$ be a Hausdorff LCA group and $0<\alpha<2 \pi / 3$. Then the pair of groups $\mathbf{G}, \widehat{\mathbf{G}}$ admits a convergent system of $\alpha$-adjoint approximation schemes. One can additionally arrange that at least one of the approximating systems $\left(\eta_{i}\right),\left(\phi_{i}\right)$ consists entirely of injective maps. 
Proof Let $\left(\left(\mathbf{Q}_{i}, \mathbf{V}_{i}\right)\right)_{i \in I},\left(\left(\boldsymbol{\Delta}_{i}, \boldsymbol{\Upsilon}_{i}\right)\right)_{i \in I}$ be some raster bases of the LCA groups $\mathbf{G}, \widehat{\mathbf{G}}$, respectively, ordered in such a way that

$$
i \leq j \Leftrightarrow\left(\mathbf{Q}_{i}, \mathbf{V}_{i}\right) \leq\left(\mathbf{Q}_{j}, \mathbf{V}_{j}\right) \text { and }\left(\boldsymbol{\Delta}_{i}, \mathbf{\Upsilon}_{i}\right) \leq\left(\boldsymbol{\Delta}_{j}, \mathbf{\Upsilon}_{j}\right)
$$

and $\left(\varepsilon_{n}\right)_{n \in \mathbb{N}}$ be a decreasing sequence of positive numbers $\varepsilon_{n}<2 \pi / 3-\alpha$ converging to 0 . According to Theorem 2.5.5, for each $i \in I$ and $n \in \mathbb{N}$, there is an $\varepsilon_{n}$-pairing preserving $\alpha$-adjoint approximation scheme given by a $\mathbf{G}-\operatorname{raster}\left(\mathbf{K}_{i n}, \mathbf{U}_{i n}\right)$, a $\widehat{\mathbf{G}}-\operatorname{raster}\left(\boldsymbol{\Gamma}_{i n}, \boldsymbol{\Omega}_{\text {in }}\right)$, a finite abelian group $G_{i n}$ and approximating maps $\eta_{i n}: G_{i n} \rightarrow \mathbf{G}, \phi_{i n}: \widehat{G}_{i n} \rightarrow \widehat{\mathbf{G}}$, compatible with the rasters $\left(\mathbf{Q}_{i}, \mathbf{V}_{i}\right),\left(\boldsymbol{\Delta}_{i}, \boldsymbol{\Upsilon}_{i}\right)$. It can be easily seen that, for all $j \geq i$, $m \geq n$, the approximation scheme given by the rasters $\left(\mathbf{K}_{j m}, \mathbf{U}_{j m}\right),\left(\boldsymbol{\Gamma}_{j m}, \boldsymbol{\Omega}_{j m}\right)$ and the approximating maps $\eta_{j m}: G_{j m} \rightarrow \mathbf{G}, \phi_{j m}: \widehat{G}_{j m} \rightarrow \widehat{\mathbf{G}}$ is $\varepsilon_{n}$-pairing preserving and compatible with $\left(\mathbf{Q}_{i}, \mathbf{V}_{i}\right),\left(\boldsymbol{\Delta}_{i}, \boldsymbol{\Upsilon}_{i}\right)$, as well. Thus $\left(\left(\boldsymbol{\Gamma}_{i n}, \boldsymbol{\Omega}_{i n}\right)\right),\left(\left(\boldsymbol{\Gamma}_{i n}, \boldsymbol{\Omega}_{i n}\right)\right)$, and $\left(\eta_{i n}\right)$, $\left(\phi_{i n}\right)$ form a convergent system of $\alpha$-adjoint approximation schemes for the pair of groups $\mathbf{G}, \widehat{\mathbf{G}}$ over the directed poset $(I \times \mathbb{N}, \leq)$ ordered by the relation $(i, n) \leq(j, m)$ if and only if $i \leq j$ and $n \leq m$. The supplement on injectivity follows from the last sentence of Theorem 2.5.5.

Remarks (a) If $\mathbf{G}$ is metrizable and $\sigma$-compact then the same is true for $\widehat{\mathbf{G}}$. In such a case the original raster bases can be indexed by the set $I=\mathbb{N}$, as well, and the double indexing of the $\alpha$-adjoint approximation schemes forming the resulting convergent system can be avoided.

(b) Similar to the proof of the implication (iv) $\Rightarrow$ (ii) in Proposition 1.3.7 (see also the subsequent remark (c)), one can obtain an adjoint pair of HFI approximations $\eta: G \rightarrow{ }^{*} \mathbf{G}, \phi: \widehat{G} \rightarrow{ }^{*} \widehat{\mathbf{G}}$ from the convergent system of $\alpha$-adjoint approximation schemes, whose existence was established in Theorem 2.5.6, by referring to the sufficient saturation of the nonstandard universe or via the ultraproduct construction applied to the approximating systems $\left(\eta_{i}: G_{i} \rightarrow \mathbf{G}\right)_{i \in I},\left(\phi_{i}: \widehat{G}_{i} \rightarrow \widehat{\mathbf{G}}\right)_{i \in I}$. The interested reader is referred to Zlatoš [49, Proposition 2.5.5, Theorem 2.5.6].

(c) The above form of Theorems 2.5.5 and 2.5.6 is not the only possible one which can be derived from Corollary 2.5.2. One could, eg, formulate the notion of compatibility with a given pair of rasters by taking care of the other two (or of all the) inclusions from Lemma 2.5.3 (b). Similarly, in Theorem 2.5.6, it is not necessary to keep the parameter $\alpha$ fixed; it would be possible to require that $\alpha>0$ becomes "arbitrarily small", and even guarantee that $\alpha=\varepsilon$. Another example of an alternative variation can be found in Zlatoš [49, Theorems 2.5.8, 2.5.9].

The "HFI parts" of the following three examples are due to Gordon [18]; we are adding the standard counterparts mainly in order to illustrate the $\alpha$-adjointness and 
the $\varepsilon$-pairing preservation phenomena. In these fairly important cases, some of the previously mentioned conditions will be satisfied automatically, in an even stricter form than required by the definitions.

In the first example, building on items (a) and (b) of Example 1.3.8, we construct certain pairs of adjoint approximations of the group $\mathbb{T}$ and its dual group $\widehat{\mathbb{T}} \cong \mathbb{Z}$, where $\gamma(\boldsymbol{x})=\boldsymbol{x}^{\gamma}$ for $\boldsymbol{x} \in \mathbb{T}, \gamma \in \mathbb{Z}$. Similarly, the (hyper)finite cyclic group $\mathbb{Z}_{n}$ is identified with its dual group $\widehat{\mathbb{Z}}_{n}$ via the pairing $\gamma(a)=\mathrm{e}^{2 \pi \mathrm{i} a \gamma / n}$, for $a, \gamma \in \mathbb{Z}_{n}$. Let us recall that $\mathbb{Z}_{n}$ is still represented as the group of absolutely smallest remainders modulo $n$.

Example 2.5.7 If $n \in{ }^{*} \mathbb{N}_{\infty}$ then the internal homomorphism $\eta: \mathbb{Z}_{n} \rightarrow{ }^{*} \mathbb{T}, \eta(a)=$ $\mathrm{e}^{2 \pi \mathrm{i} a / n}$, and the inclusion map $\phi: \mathbb{Z}_{n} \rightarrow{ }^{*} \mathbb{Z}$ give rise to the mutually dual IMG group triplets $\left(\mathbb{Z}_{n}, G_{0}, \mathbb{Z}_{n}\right),\left(\mathbb{Z}_{n},\{0\}, \mathbb{Z}\right)$, with normalizing multipliers $d=n^{-1}, \widehat{d}=1$, respectively, where:

$$
G_{0}=\left\{a \in \mathbb{Z}_{n}: \frac{a}{n} \approx 0\right\}=\mathbb{Z}^{\downarrow}
$$

For $a, \gamma \in \mathbb{Z}_{n}$ we even have

$$
(\phi \gamma)(\eta a)=\left(\mathrm{e}^{\frac{2 \pi \mathrm{i} a}{n}}\right)^{\gamma}=\mathrm{e}^{\frac{2 \pi \mathrm{i} a \gamma}{n}}=\gamma(a)
$$

showing that $\eta, \phi$ strictly preserve the pairing on the mutually dual groups $\mathbb{T}, \mathbb{Z}$.

Passing to the standard situation, let $n, k \in \mathbb{N}$ and $0<r \leq \alpha<2 \pi / 3$ be such that $n r>\pi$ and $1 \leq k<n / 4$. We put:

$$
\mathbf{U}=\{\boldsymbol{x} \in \mathbb{T}:|\arg \boldsymbol{x}| \leq r\} \quad \Gamma=\{\gamma \in \mathbb{Z}:|\gamma| \leq k\}
$$

Then we have $\operatorname{Bohr}_{\alpha}(\mathbb{T})=\{0\}$ and

$$
\operatorname{Bohr}_{\alpha}(\Gamma)=\left\{\boldsymbol{x} \in \mathbb{T}:|\arg \boldsymbol{x}| \leq \frac{\alpha}{k}\right\}
$$

hence the $\mathbb{T}$-raster $(\mathbb{T}, \mathbf{U})$ and the $\mathbb{Z}$-raster $(\Gamma,\{0\})$ are $\alpha$-adjoint if and only if $r k \leq \alpha$. The $(\mathbb{T}, \mathbf{U})$ approximation $\eta: \mathbb{Z}_{n} \rightarrow \mathbb{T}$ and the $(\Gamma,\{0\})$ approximation $\phi: \mathbb{Z}_{n} \rightarrow \mathbb{Z}$ (both given as in the HFI case) still satisfy $(\phi \gamma)(\eta a)=\gamma(a)$ for $a, \gamma \in \mathbb{Z}_{n}$, so that they are even pairing preserving on $\mathbb{T} \times \Gamma$.

Assume that $(\mathbb{T}, \mathbf{V})$ is a $\mathbf{G}$-raster and $(\Delta,\{0\})$ is a $\mathbb{Z}$-raster given in advance, where:

$$
\mathbf{V}=\{\boldsymbol{x} \in \mathbb{T}:|\arg \boldsymbol{x}| \leq s\} \quad \Delta=\{\gamma \in \mathbb{Z}:|\gamma| \leq m\}
$$

Then $(\mathbb{T}, \mathbf{V}) \leq(\mathbb{T}, \mathbf{U})$ if and only if $r \leq s$, and similarly, $(\Delta,\{0\}) \leq(\Gamma,\{0\})$ if and only if $m \leq k$. A simple computation shows that

$$
\operatorname{Bohr}_{\alpha}\left(\eta^{-1}[\mathbf{V}]\right)=\left\{\gamma \in \mathbb{Z}_{n}:|\gamma| \leq \frac{\alpha}{s}\right\} \quad \text { and } \quad \phi^{-1}[\Gamma]=\left\{\gamma \in \mathbb{Z}_{n}:|\gamma| \leq k\right\}
$$


while $\operatorname{Bohr}_{\alpha}\left(\phi^{-1}[0]\right)=\mathbb{Z}_{n}=\eta^{-1}[\mathbb{T}]$. Thus the approximation scheme given by the rasters $(\mathbb{T}, \mathbf{U}),(\Gamma,\{0\})$ and mappings $\eta: \mathbb{Z}_{n} \rightarrow \mathbb{T}, \phi: \mathbb{Z}_{n} \rightarrow \mathbb{Z}$ is compatible with the rasters $(\mathbb{T}, \mathbf{V}),(\Delta,\{0\})$ if and only if $\lfloor\alpha / s\rfloor \leq k$. All the needed conditions can be guaranteed by taking $k$ and $n$ big and $r$ small enough.

In the next example we describe a pair of adjoint approximations for the self-dual group $\mathbb{R}$, building on item (c) of Example 1.3.8. More precisely, $\mathbb{R}$ is identified with its dual group $\widehat{\mathbb{R}}$ via the pairing $\gamma(\boldsymbol{x})=\mathrm{e}^{\mathrm{i} x \boldsymbol{\gamma}}$, for $\boldsymbol{x}, \boldsymbol{\gamma} \in \mathbb{R}$. The passage to differently scaled pairings $\gamma(\boldsymbol{x})=\mathrm{e}^{2 \pi \mathrm{i} x \boldsymbol{\gamma} / T}$, with any $T>0$, is straightforward.

Example 2.5.8 Let $n \in{ }^{*} \mathbb{N}_{\infty}$, and $d, d^{\prime}$ be positive infinitesimals such that both the hyperreal numbers $n d, n d^{\prime}$ are infinite. Then the internal mappings $\eta: \mathbb{Z}_{n} \rightarrow{ }^{*} \mathbb{R}$, $\eta(a)=a d$, and $\phi: \mathbb{Z}_{n} \rightarrow{ }^{*} \mathbb{R}, \phi(\gamma)=\gamma d^{\prime}$, are HFI approximations of the group $\mathbb{R}$, inducing IMG group triplets $\left(\mathbb{Z}_{n}, G_{0}, G_{\mathrm{f}}\right),\left(\mathbb{Z}_{n}, H_{0}, H_{\mathrm{f}}\right)$, where

$$
\begin{array}{ll}
G_{0}=\left\{a \in \mathbb{Z}_{n}: a d \approx 0\right\} & G_{\mathrm{f}}=\left\{a \in \mathbb{Z}_{n}:|a| d<\infty\right\} \\
H_{0}=\left\{\gamma \in \mathbb{Z}_{n}: \gamma d^{\prime} \approx 0\right\} & H_{\mathrm{f}}=\left\{\gamma \in \mathbb{Z}_{n}:|\gamma| d^{\prime}<\infty\right\}
\end{array}
$$

with normalizing multipliers $d, d^{\prime}$, respectively. One can easily verify that these triplets are mutually dual if and only if $n d d^{\prime} \in \mathbb{F}^{*} \mathbb{R} \backslash \mathbb{I}^{*} \mathbb{R}$. For any $a, \gamma \in \mathbb{Z}_{n}$ we have

$$
(\phi \gamma)(\eta a)=\mathrm{e}^{\mathrm{i} a \gamma d d^{\prime}} \quad \gamma(a)=\mathrm{e}^{\frac{2 \pi \mathrm{i} a \gamma}{n}}
$$

and the two expressions are infinitesimally close for all $a \in G_{\mathrm{f}}, \gamma \in H_{\mathrm{f}}$ if and only if:

$$
n d d^{\prime} \approx 2 \pi
$$

Hence this condition is equivalent to the adjointness of the HFI approximations $\eta, \phi$. Then the normalizing coefficient $\widehat{d}=1 / n d$, dual to $d$, is a normalizing multiplier for the triplet $\left(\mathbb{Z}_{n}, H_{0}, H_{\mathrm{f}}\right)$, as well. Under the particular choice

$$
d^{\prime}=2 \pi \widehat{d}=\frac{2 \pi}{n d}
$$

we even have $(\phi \gamma)(\eta a)=\gamma(a)$ for all $a, \gamma \in \mathbb{Z}_{n}$.

The corresponding standard situation is described in terms of some $n, k, m \in \mathbb{N}$ and positive $d, d^{\prime}, r, \rho, \alpha, \varepsilon \in \mathbb{R}$, such that $1 \leq k, m<n / 4, \alpha+\varepsilon<2 \pi / 3$, and $d / 2<r \leq k d, d^{\prime} / 2<\rho \leq m d^{\prime}$. We put

$$
\begin{array}{ll}
\mathbf{U}=[-r, r] & \mathbf{K}=[-k d, k d] \\
\mathbf{\Omega}=[-\rho, \rho] & \boldsymbol{\Gamma}=\left[-m d^{\prime}, m d^{\prime}\right]
\end{array}
$$


while the approximating mappings $\eta: \mathbb{Z}_{n} \rightarrow \mathbb{R}, \phi: \mathbb{Z}_{n} \rightarrow \mathbb{R}$ are given by the same formulas as in the HFI case. Then

$$
\operatorname{Bohr}_{\alpha}(\mathbf{K})=\left[-\frac{\alpha}{k d}, \frac{\alpha}{k d}\right] \quad \text { and } \quad \operatorname{Bohr}_{\alpha}(\boldsymbol{\Gamma})=\left[-\frac{\alpha}{m d^{\prime}}, \frac{\alpha}{m d^{\prime}}\right]
$$

thus the $\mathbb{R}$-rasters $(\mathbf{K}, \mathbf{U}),(\boldsymbol{\Gamma}, \boldsymbol{\Omega})$ are $\alpha$-adjoint if and only if both $r \cdot m d^{\prime} \leq \alpha$ and $\rho \cdot k d \leq \alpha$. The reversed proportionality of the lengths $r$ and $m d^{\prime}$, as well as that of $\rho$ and $k d$ is worth noticing.

For any $a, \gamma \in \mathbb{Z}_{n}$ we have:

$$
\left|\arg \frac{(\phi \gamma)(\eta a)}{\gamma(a)}\right|=|a \gamma| d d^{\prime}\left|1-\frac{2 \pi}{n d d^{\prime}}\right|
$$

This expression is $\leq \varepsilon$ for all $a \in \eta^{-1}[\mathbf{K}], \gamma \in \phi^{-1}[\Gamma]$ if and only if:

$$
k m d d^{\prime}\left|1-\frac{2 \pi}{n d d^{\prime}}\right| \leq \varepsilon
$$

Putting $d^{\prime}=2 \pi / n d$, we can even achieve that $(\phi \gamma)(\eta a)=\gamma(a)$ for all $a, \gamma \in \mathbb{Z}_{n}$.

Assume that $(\mathbf{Q}, \mathbf{V}),(\boldsymbol{\Delta}, \boldsymbol{\Upsilon})$ are two $\mathbb{R}$-rasters given in advance, where $\mathbf{V}=[-s, s]$ and $\Upsilon=[-\sigma, \sigma]$. By choosing $r, \rho$ small and $k, m$ big enough we can always guarantee that $(\mathbf{Q}, \mathbf{V}) \leq(\mathbf{K}, \mathbf{U})$ and $(\Delta, \Upsilon) \leq(\boldsymbol{\Gamma}, \boldsymbol{\Omega})$, thus in particular we need not care about a more detailed description of $\mathbf{Q}$ and $\Delta$.

One can easily verify that:

$$
\begin{array}{ll}
\operatorname{Bohr}_{\alpha}\left(\eta^{-1}[\mathbf{V}]\right)=\left\{\gamma \in \mathbb{Z}_{n}:|\gamma| \leq \frac{n d \alpha}{2 \pi s}\right\} & \phi^{-1}[\boldsymbol{\Gamma}]=\left\{\gamma \in \mathbb{Z}_{n}:|\gamma| \leq m\right\} \\
\operatorname{Bohr}_{\alpha}\left(\phi^{-1}[\Upsilon]\right)=\left\{a \in \mathbb{Z}_{n}:|a| \leq \frac{n d^{\prime} \alpha}{2 \pi \sigma}\right\} & \eta^{-1}[\mathbf{K}]=\left\{a \in \mathbb{Z}_{n}:|a| \leq k\right\}
\end{array}
$$

Hence the inclusions $\operatorname{Bohr}_{\alpha}\left(\eta^{-1}[\mathbf{V}]\right) \subseteq \phi^{-1}[\boldsymbol{\Gamma}]$ and $\operatorname{Bohr}_{\alpha}\left(\eta^{-1}[\Omega]\right) \subseteq \phi^{-1}[\mathbf{K}]$ are equivalent to the inequalities

$$
\left\lfloor\frac{n d \alpha}{2 \pi s}\right\rfloor \leq m \quad \text { and } \quad\left\lfloor\frac{n d^{\prime} \alpha}{2 \pi \sigma}\right\rfloor \leq k
$$

respectively. In particular, if $n d d^{\prime}=2 \pi$, they reduce to $\left\lfloor\alpha / s d^{\prime}\right\rfloor \leq m$ and $\lfloor\alpha / \sigma d\rfloor \leq k$.

Now, it is routine to check that, for any $\alpha>0, \varepsilon \geq 0$, subject to $\alpha+\varepsilon<2 \pi / 3$, one can always construct an $\alpha$-adjoint $\varepsilon$-pairing preserving approximation scheme for the selfdual group $\mathbb{R}$ of the form just described, compatible with arbitrary $\mathbb{R}$-rasters $(\mathbf{Q}, \mathbf{V}),(\boldsymbol{\Delta}, \mathbf{\Upsilon})$ given in advance, simply by choosing the parameters $n, k, m, r, \rho$ and $d, d^{\prime}$ properly. 
In the final example we construct adjoint approximations to those from item (d) of Example 1.3.8.

Example 2.5.9 Let $\mathbf{G}$ be a Hausdorff LCA group with a raster base $\left(\left(\mathbf{K}_{i}, \mathbf{U}_{i}\right)\right)_{i \in I}$ consisting of compact open subgroups $\mathbf{U}_{i} \subseteq \mathbf{K}_{i}$ of $\mathbf{G}$. As Bohr $_{\alpha}(\mathbf{X})$ coincides with the annihilator $\mathbf{X}^{\perp}=\operatorname{Bohr}_{0}(\mathbf{X})$ for $\alpha \in(0,2 \pi / 3)$ and any subgroup $\mathbf{X} \subseteq \mathbf{G}$, the system $\left(\left(\mathbf{U}_{i}^{\perp}, \mathbf{K}_{i}^{\perp}\right)\right)_{i \in I}$ forms a raster base of the dual group $\widehat{\mathbf{G}}$ consisting of compact open subgroups, again. Picking $i \in I$ and putting $\mathbf{U}=\mathbf{U}_{i}, \mathbf{K}=\mathbf{K}_{i}$ and $\boldsymbol{\Omega}=\mathbf{K}^{\perp}, \Gamma=\mathbf{U}^{\perp}$, it is obvious that the pairs $(\mathbf{K}, \mathbf{U}),(\boldsymbol{\Gamma}, \boldsymbol{\Omega})$ are $\alpha$-adjoint for each $\alpha \in(0,2 \pi / 3)$. The quotients $G=\mathbf{K} / \mathbf{U}$ and $\Gamma / \boldsymbol{\Omega}$ are finite abelian groups and the latter can be canonically identified with the dual group $\widehat{G}$. Any right inverse map $\eta: G \rightarrow \mathbf{K} \subseteq \mathbf{G}$ to the canonical projection $\zeta: \mathbf{K} \rightarrow \mathbf{K} / \mathbf{U}$ is a $(\mathbf{K}, \mathbf{U})$ approximation of $\mathbf{G}$, and similarly, any right inverse map $\phi: \widehat{G} \rightarrow \boldsymbol{\Gamma} \subseteq \widehat{\mathbf{G}}$ to the canonical projection $\xi: \Gamma \rightarrow \Gamma / \Omega$ is a $(\boldsymbol{\Gamma}, \boldsymbol{\Omega})$ approximation of $\widehat{G}$. Then one can easily verify by a straightforward computation that, for any $a \in G, \gamma \in \widehat{G}$ :

$$
(\phi \gamma)(\eta a)=\gamma(a)
$$

Finally, the equalities

$$
\widehat{G}=\operatorname{Bohr}_{\alpha}\left(\eta^{-1}[\mathbf{U}]\right)=\phi^{-1}[\mathbf{\Gamma}] \quad G=\operatorname{Bohr}_{\alpha}\left(\phi^{-1}[\Omega]\right)=\eta^{-1}[\mathbf{K}]
$$

show that the approximation scheme given by the $\alpha$-adjoint rasters $(\mathbf{K}, \mathbf{U}),(\boldsymbol{\Gamma}, \boldsymbol{\Omega})$ and the mappings $\eta, \phi$ is compatible with these very rasters, hence it is a pairing preserving $\alpha$-adjoint approximation scheme for the pair $\mathbf{G}, \widehat{\mathbf{G}}$.

If $\mathbf{U} \subseteq \mathbf{K}$ are ${ }^{*}$ compact ${ }^{*}$ open subgroups of ${ }^{*} \mathbf{G}$, such that $\mathbf{U} \subseteq \mathbb{I}^{*} \mathbf{G}, \mathbb{F}^{*} \mathbf{G} \subseteq \mathbf{K}$ then $\boldsymbol{\Omega}=\mathbf{K}^{\downarrow}=\mathbf{K}^{\perp}, \Gamma=\mathbf{U}^{\downarrow}=\mathbf{U}^{\perp}$ are ${ }^{*}$ compact ${ }^{*}$ open subgroups of ${ }^{*} \widehat{\mathbf{G}}$, such that $\Omega \subseteq \mathbb{I}^{*} \widehat{\mathbf{G}}, \mathbb{F}^{*} \widehat{\mathbf{G}} \subseteq \Gamma$. The quotients $G=\mathbf{K} / \mathbf{U}, \widehat{G}=\Gamma / \Omega$ are mutually dual hyperfinite abelian groups. The HFI approximations $\eta: G \rightarrow{ }^{*} \mathbf{G}, \phi: \widehat{G} \rightarrow{ }^{*} \widehat{\mathbf{G}}$ can be constructed in essentially the same way as in the standard situation above. The corresponding IMG group triplets $\left(G, G_{0}, G_{\mathrm{f}}\right),\left(\widehat{G}, H_{0}, H_{\mathrm{f}}\right)$, where

$$
\begin{aligned}
G_{0} & =\eta^{-1}\left[\mathbb{I}^{*} \mathbf{G}\right] & & G_{\mathrm{f}}=\eta^{-1}\left[\mathbb{F}^{*} \mathbf{G}\right] \\
H_{0} & =\phi^{-1}[\mathbb{I} * \widehat{\mathbf{G}}] & & H_{\mathrm{f}}=\phi^{-1}\left[\mathbb{F}^{*} \widehat{\mathbf{G}}\right]
\end{aligned}
$$

are mutually dual, as well. For any internal subgroup $\mathbf{X}$ of $\mathbf{G}$, such that $\mathbb{I}^{*} \mathbf{G} \subseteq \mathbf{X} \subseteq$ $\mathbb{F}^{*} \mathbf{G}, d=[\mathbf{X}: \mathbf{U}]^{-1}$ and $\widehat{d}=(|G| d)^{-1}=[\mathbf{K}: \mathbf{X}]^{-1}$ can serve as adjoint normalizing multipliers for the triplets $\left(G, G_{0}, G_{\mathrm{f}}\right)$ and $\left(\widehat{G}, H_{0}, H_{\mathrm{f}}\right)$, respectively. The equality $(\phi \gamma)(\eta a)=\gamma(a)$ holds even for all $a \in G, \gamma \in \widehat{G}$, while $(\phi \gamma)(\eta a) \approx \gamma(a)$ for $a \in G_{\mathrm{f}}$, $\gamma \in H_{\mathrm{f}}$ would be enough to establish the adjointness of $\eta, \phi$. 
In Gordon [18], pairs of adjoint HFI approximations for $\tau$-adic solenoids $\Sigma_{\tau}$ and their dual groups of $\tau$-adic rationals

$$
\mathbb{Q}^{(\tau)}=\bigcup_{n \in \mathbb{N}}\left(\frac{1}{\tau_{n}}\right) \mathbb{Z}=\left\{\frac{a}{\tau_{n}}: a \in \mathbb{Z} \& n \in \mathbb{N}\right\}
$$

are also described. These, as well as the corresponding finite approximations, can be constructed combining some ideas from Examples 2.5.7-2.5.9.

In view of the supplements on injectivity after Corollary 2.5.2 and in Theorems 2.5.5 and 2.5.6, as well as of the last three examples, it is natural to formulate the following conjecture. We prefer to state it in the more concise nonstandard form. The standard translation can be readily obtained from Theorem 2.5.5.

Conjecture Let $\mathbf{G}$ be a Hausdorff LCA group. Then there exists a hyperfinite abelian group $G$ and injective mappings $\eta: G \rightarrow{ }^{*} \mathbf{G}, \phi: \widehat{G} \rightarrow{ }^{*} \widehat{\mathbf{G}}$ forming an adjoint pair of HFI approximations of the mutually dual groups $\mathbf{G}, \widehat{\mathbf{G}}$, respectively.

Let us conclude by a brief discussion of the roles of standard and nonstandard methods in the proof of the Adjoint Approximation Scheme Theorem 2.5.5. Our starting point was the proof of the existence of arbitrarily good (standard) finite approximations for any single LCA group $\mathbf{G}$ (Theorem 1.3.10) from which we derived the existence of (nonstandard) hyperfinite approximations of $\mathbf{G}$ by means of the Transfer Principle (Corollary 1.3.11). Next we proved another nonstandard result, namely the existence of an adjoint approximation to any HFI approximation of $\mathbf{G}$ (Theorem 2.5.1 and Corollary 2.5.2). Both in their formulation and proof the (inherently nonstandard) Gordon's Conjecture 1 (Theorem 2.2.4) and the Triplet Duality Theorem 2.2.5 were crucial. Finally we turned back, deriving the existence of an arbitrarily precise pairing preserving adjoint pair of (standard) finite approximations of the mutually dual groups $\mathbf{G}$, $\widehat{\mathbf{G}}$ (Theorem 2.5.5) from the nonstandard Corollary 2.5.2, using the Transfer Principle in the "opposite direction".

\section{References}

[1] S Albeverio, J E Fenstad, R Høegh-Krohn, T Lindstrøm, Nonstandard Methods in Stochastic Analysis and Mathematical Physics, Academic Press, London-New York-Orlando (1986)

[2] M A Alekseev, L Y Glebsky, E I Gordon, On approximations of groups, group actions and Hopf algebras, J. Math. Sci. 107 (2001) 4305-4332; https://doi.org/10.1023/ A:1012485910692 
[3] R M Anderson, Star-finite representations of measure spaces, Trans. Amer. Math. Soc. 271 (1982) 667-687; https://doi.org/10.1090/S0002-9947-1982-0654856-1

[4] L O Arkeryd, N J Cutland, C W Henson, (eds), Nonstandard Analysis, Theory and Applications, Kluwer Academic Publishers, Dordrecht-Boston-London (1997)

[5] E Breuillard, B Green, T Tao, The structure of approximate groups, Publ. Math. IHÉS 116 (2012) 115-221; https://doi.org/10.1007/s10240-012-0043-9

[6] C C Chang, H J Keisler, Model Theory, (3rd ed.) edition, North-Holland, Amsterdam (1990)

[7] NJ Cutland, Nonstandard measure theory and its applications, Bull. London Math. Soc. 15 (1983) 529-589; https://doi.org/10.1112/blms/15.6.529

[8] M Davis, Applied Nonstandard Analysis, John Wiley \& Sons, New York-London (1977)

[9] D Dikranjan, L Stoyanov, An elementary approach to Haar integration and Pontryagin duality in locally compact abelian groups, Topology and Its Applications 158 (2011) 1942-1961; https://doi.org/10.1016/j.topol.2011.06.037

[10] R Engelking, General Topology, PWN - Polish Scientific Publishers, Warsaw (1990)

[11] CL Epstein, How well does the finite Fourier transform approximate the Fourier transform?, Communications Pure Appl. Math. 58 (2005) 1-15; https://doi.org/10.1002/ cpa. 20064

[12] L Y Glebsky, E I Gordon, On approximations of topological groups by finite quasigroups and finite semigroups, Illinois J. Math. 49 (2005) 1-16

[13] L Y Glebsky, E I Gordon, C J Rubio, On approximations of unimodular groups by finite quasigroups, Illinois J. Math. 49 (2005) 17-31

[14] R Goldblatt, Lectures on the Hyperreals, An Introduction to Nonstandard Analysis, Springer, New York-Berlin-Heidelberg (1998)

[15] I Goldbring, Hilbert's fifth problem for local groups, Ann. of Math. 172 (2010) 1269-1314

[16] I Goldbring, Nonstandard hulls of locally uniform groups, Fund. Math. 220 (2013) 93-118

[17] E I Gordon, Nonstandard analysis and locally compact abelian groups, Acta Appl. Math. 25 (1991) 221-239; https://doi.org/10.1007/BF00047004

[18] E I Gordon, Nonstandard Methods in Commutative Harmonic Analysis, volume 164, Translations of Mathematical Monographs, Amer. Math. Soc., Providence, R. I. (1997)

[19] E I Gordon, A M Vershik, Groups that are locally embeddable in the class of finite groups, St. Petersburg Math. J. 9 (1998) 49-67

[20] B Green, I Ruzsa, Freiman's theorem in an arbitrary abelian group, J. London Math. Soc. (2) 75 (2007) 163-175; https://doi.org/10.1112/jlms/jdl021

[21] K Gröchenig, Foundations of Time-Frequency Analysis, Birkhäuser, Boston-BaselBerlin (2001) 
[22] C W Henson, The nonstandard hulls of a uniform space, Pacific. J. Math. 43 (1972) 115-137; https://doi.org/10.2140/pjm.1972.43.115

[23] CW Henson, Foundations of nonstandard analysis: A gentle introduction to nonstandard extensions, from: "Nonstandard Analysis, Theory and Applications", (L O Arkeryd, N J Cutland, C W Henson, editors), Kluwer Academic Publishers, DordrechtBoston-London (1997) 1-49

[24] E Hewitt, KA Ross, Abstract Harmonic Analysis I, Springer, Berlin-GötingenHeidelberg (1963)

[25] E Hewitt, K A Ross, Abstract Harmonic Analysis II, Springer, Berlin-Heidelberg-New York (1970)

[26] E Hrushovski, Stable group theory and approximate subgroups, J. Amer. Math. Soc. 25 (2012) 189-243; https://doi.org/10.1090/S0894-0347-2011-00708-X

[27] D Landers, L Rogge, Universal Loeb measurability of sets and of the standard part map with applications, Trans. Amer. Math. Soc. 304 (1987) 229-243; https://doi.org/10.1090/S0002-9947-1987-0906814-1

[28] P A Loeb, Conversion from nonstandard to standard measure spaces and applications to probability theory, Trans. Amer. Math. Soc. 211 (1975) 113-122; https://doi.org/10.1090/S0002-9947-1975-0390154-8

[29] P A Loeb, Nonstandard analysis and topology, from: "Nonstandard Analysis, Theory and Applications", (L O Arkeryd, N J Cutland, C W Henson, editors), Kluwer Academic Publishers, Dordrecht-Boston-London (1997) 77-89

[30] L H Loomis, An Introduction to Abstract Harmonic Analysis, D. Van Nostrand, Princeton, N J (1953)

[31] W A J Luxemburg, A general theory of monads, from: "Applications of Model Theory to Algebra, Analysis and Probability, Proceedings of an International Symposium on Non-Standard Analysis", (W A J Luxemburg, editor), Hort and Reinhart and Winston, New York (1969) 18-86

[32] W A J Luxemburg, A nonstandard analysis approach to Fourier analysis, from: "Contributions to Non-Standard Analysis", (W A J Luxemburg, A Robinson, editors), North-Holland, Amsterdam-London (1972) 15-39

[33] M Mačaj, P Zlatoš, Approximate extension of partial $\varepsilon$-characters of abelian groups to characters with application to integral point lattices, Indag. Math. 16 (2005) 237-250; https://doi.org/10.1016/S0019-3577(05)80026-6

[34] J Mlček, P Zlatoš, The Ramsey structure of A-determined sets in a $\kappa$-saturated universe, from: "Proc. Logic Colloquium, Prague '98, Lecture Notes in Logic", (S R Buss, P Hájek, P Pudlák, editors), volume 13, Association for Symbolic Logic, A. K. Peters, Nattick, Mass (1998) 316-333

[35] J Mlček, P Zlatoš, Some Ramsey type theorems for countably determined sets, Arch. Math. Logic 41 (2002) 619-630; https://doi.org/10.1007/s001530100129 
[36] S A Morris, Pontryagin Duality and the Structure of Locally Compact Abelian Groups, Cambridge University Press, London-New York-Melbourne (1977)

[37] E Nelson, The syntax of nonstandard analysis, Annals of Pure and Applied Logic 38 (1988) 123-134; https://doi.org/10.1016/0168-0072(88)90050-4

[38] LS Pontryagin, Nepreryvnye gruppy, 4 edition, Nauka, Moskva (1984)(Russian); English translation, Topological Groups, Gordon \& Breach, New York, 1986

[39] A Robinson, Non-Standard Analysis, revised edition, Princeton University Press, Princeton, N J (1996)

[40] J Roe, Lectures on Coarse Geometry, University Lecture Series, volume 31, revised edition, Amer. Math. Soc., Providence, R I (2003)

[41] D A Ross, Loeb measure and probability, from: "Nonstandard Analysis, Theory and Applications", (L O Arkeryd, N J Cutland, C W Henson, editors), Kluwer Academic Publishers, Dordrecht-Boston-London (1997) 91-120

[42] W Rudin, Fourier Analysis on Groups, Interscience, John Wiley \& Sons, New YorkLondon (1962)

[43] T Tao, Structure and Randomness, Amer. Math. Soc., Providence, R I (2008)

[44] T Tao, V Vu, Additive Combinatorics, Cambridge University Press, Cambridge-New York, etc (2006)

[45] A Terras, Fourier Analysis on Finite Groups and Applications, Cambridge University Press, Cambridge-New York, etc (1999, reprinted 2001)

[46] A M Turing, Finite approximations to Lie groups, Ann. of Math. 39 (1938) 105-111

[47] M Ziman, P Zlatoš, Hyperfinite dimensional representations of spaces and algebras of measures, Monatsh. Math. 148 (2006) 241-261; https://doi.org/10.1007/s00605-0050375-3

[48] P Zlatoš, Stability of group homomorphisms in the compact-open topology, J. Logic \& Analysis 2:3 (2010) 1-15; https://doi.org/10.4115/jla.2010.2.3

[49] P Zlatoš, Gordon's Conjectures: Pontryagin-van Kampen duality and Fourier transform in the hyperfinite setting (2019); arXiv:1409.6128

[50] P Zlatoš, Gordon's Conjecture 3: The Fourier transform in the hyperfinite setting, Journal of Logic and Analysis (to appear)

Faculty of Mathematics, Physics and Informatics, Comenius University, 84248 Bratislava, Slovakia

zlatos@fmph.uniba.sk

Received: 3 July 2015 Revised: 4 January 2021 